\title{
The Taurus Boundary of Stellar/Substellar (TBOSS) Survey
}

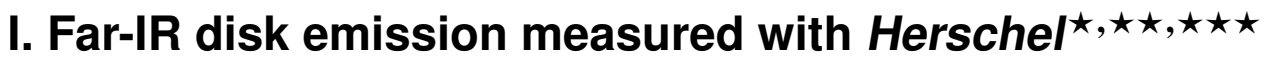

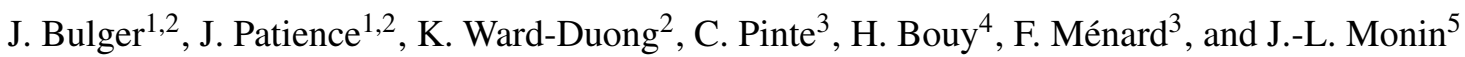 \\ 1 School of Physics, University of Exeter, Exeter, EX4 4QL, UK \\ e-mail: joanna@astro.ex.ac.uk \\ 2 School of Earth and Space Exploration, Arizona State University, Tempe, AZ 85281, USA \\ e-mail: jbulger1@asu.edu \\ 3 Universidad de Chile, Camino El Observatorio 1515, Casilla 36-D Las Condes, Santiago, Chile \\ ${ }^{4}$ Centro de Astrobiología - Depto. Astrofísica (CSIC- INTA), ESAC Campus, PO Box 78, 28691 Villanueva de la Cañada, Spain \\ 5 UJF-Grenoble 1/CNRS-INSU, Institut de Planétologie et d'Astrophysique de Grenoble (IPAG) UMR 5274, 38041 Grenoble, France
}

Received 19 November 2013 / Accepted 2 July 2014

\begin{abstract}
With the PACS instrument on Herschel, 134 low mass members of the Taurus star-forming region spanning the M4-L0 spectral type range and covering the transition from low mass stars to brown dwarfs were observed. Combining the new Herschel results with other Herschel programs, a total of 150 of the 154 M4-L0 Taurus members have observations, and we have added an additional 3 targets from Spitzer to form the 153-object TBOSS (Taurus Boundary of Stellar/Substellar) sample, a 99\% complete study. Among the 150 targets, $70 \mu \mathrm{m}$ flux densities were measured for 7 of the 7 Class I objects, 48 of the 67 Class II objects, and 3 of the 76 Class III objects. For the detected Class II objects, the median $70 \mu \mathrm{m}$ flux density level declines with spectral type; however, the distribution of excess relative to central object flux density does not change across the stellar/substellar boundary in the M4-L0 range. Connecting the $70 \mu \mathrm{m}$ TBOSS values with the results from K0-M3 Class II members results in the first comprehensive census of far-IR emission across the full mass spectrum of the stellar and substellar population of a star-forming region, and the median flux density declines with spectral type in a trend analogous to the flux density decline expected for the central objects. Spectral energy distributions (SEDs) were constructed for all TBOSS targets covering the optical to far-IR range and extending to the submm/mm for a subset of sources with longer wavelength data. Based on an initial exploration of the impact of different physical parameters on the Herschel flux densities, geometrical factors such as inclination and structural parameters such as scale height and flaring have the largest influence on the flux densities in the PACS bands. From the $24 \mu \mathrm{m}$ to $70 \mu \mathrm{m}$ spectral index of the SEDs, 5 new candidate transition disks were identified. Considering the previously known and new candidate transition disks, the spectral indices over longer wavelengths $(\geq 70 \mu \mathrm{m})$ are not distinct from those of the full Class II population, suggesting that the outer regions of the transition disks are similar to Class II disks. The steep $24 \mu \mathrm{m}$ to $70 \mu \mathrm{m}$ slope for a subset of 8 TBOSS targets may be an indication of truncated disks in these systems, however additional measurements are required to establish the outer radii of these disks conclusively. From existing high angular resolution companion search observations, two examples of mixed pair systems that include secondaries with disks were measured in the Herschel data. Finally, comparing the TBOSS results with a Herschel study of Ophiuchus brown dwarfs reveals a lower fraction of disks around the Taurus substellar population with flux densities comparable to the Ophiuchus disks.
\end{abstract}

Key words. brown dwarfs - stars: pre-main sequence - protoplanetary disks

\section{Introduction}

Disks are critical structures in the star and planet formation process, as they provide a conduit to channel material onto the central object (e.g., Hartmann 1997) and supply a reservoir of dust and gas to form planets (e.g., Pollack et al. 1996; Boss 1997). Variations in the frequency, lifetimes, and structure of disks as a function of central object mass have important implications for formation models (e.g., Bate et al. 2003; Reipurth $\&$ Clarke 2001) and the viability of future planet formation.

\footnotetext{
* Herschel is an ESA space observatory with science instrument provided by European-led Principal Investigator consortia, and with important participation from NASA.

$\star \star$ Appendices are available in electronic form at

http: //www . aanda.org

$\star \star \star$ Tables 1, 2, 4, 5, and A.1-A.4 are only available at the CDS via anonymous ftp to cdsarc.u-strasbg. fr (130.79.128.5) or via http://cdsarc.u-strasbg.fr/viz-bin/qcat?]/A+A/570/A29
}

Infrared and mid-infrared observations of young brown dwarfs (e.g., Luhman 2006; Luhman et al. 2010; Guieu et al. 2007) have enabled the identification of inner disks. This common presence of disks around stellar and substellar objects has been used to argue for a common formation mechanism (Scholz \& Jayawardhana 2008; Haisch et al. 2010), while other observations hint at possible differences in the dynamical history of low mass stars/brown dwarfs compared to higher mass stars (Thies \& Kroupa 2007). Formation scenarios for brown dwarfs also have implications for the properties of brown dwarf disks, and a number of possible mechanisms have been proposed, including ejection of stellar embryos (e.g., Bate et al. 2003; Reipurth \& Clarke 2001), erosion of star-forming clouds by radiation from massive stars (Whitworth \& Zinnecker 2004), gravitational instabilities in the early stages of disks (e.g., Stamatellos \& Whitworth 2011; Basu \& Vorobyov 2012), and gravitational collapse of clouds analogous to the formation of stars (e.g., Padoan et al. 2005; Hennebelle \& Chabrier 2008). 
Trends in the population of exoplanets orbiting low mass stars have suggested intriguing differences compared to the planetary systems around more massive stars and observations of protoplanetary disks around low mass objects are required to investigate possible origins of the distinct populations. Analysis of the Kepler planet candidate statistics shows a steep increase in the occurrence of planets with the smallest radii as host star mass declines (Howard et al. 2012). By contrast, the frequency of hot Jupiters around M-stars is lower than that for higher mass stars (e.g., Johnson et al. 2010). The detection of brown dwarfs with directly-imaged planetary mass companions (Chauvin et al. 2004; Song et al. 2006) indicate that at least some substellar objects are associated with planet formation early in their history. The overall exoplanet population frequency remains unclear around brown dwarfs. By detecting and characterizing the disks around young M-stars and brown dwarfs, it is possible to investigate the origins of the exoplanet populations.

As one of the nearest regions of star-formation $(\sim 140 \mathrm{pc}$; Kenyon et al. 1994; Bertout et al. 1999; Torres et al. 2009), Taurus represents an important population of young stars and brown dwarfs for which detailed investigations of disk frequencies and properties are possible. The stellar density of the Taurus region is exceptionally low compared to other star-forming regions based on a calculation from the most recent membership studies and a consistent approach (King et al. 2012a,b). This low density enables isolating the individual objects and avoiding confusion within the beam of longer wavelength observations. Large-scale surveys of Taurus members have identified the presence of disks based on excess emission above the photosphere out to $24 \mu \mathrm{m}$ with sensitive Spitzer measurements (e.g., Luhman et al. 2010), but these wavelengths do not probe the cooler material in the disk that covers the portions of the spectral energy distributions (SEDs) over which the emission transitions from optically thick to optically thin, and is strongly influenced by factors such as flaring, scale height, and settling. The Spitzer survey of Taurus at $70 \mu \mathrm{m}$ (Rebull et al. 2010) detected a large population of the earlier spectral type members, but did not have the sensitivity to measure disk emission from the lowest mass stars and brown dwarfs. In Taurus, $\sim 50 \%$ of the known members have spectral types later than M3 (Luhman et al. 2010), making the existing far-IR measurements fundamentally incomplete and resulting in a biased view of the early stages of star and planet formation. As pre-Main Sequence stars contract onto the Main Sequence, their effective temperatures increase and a spectral type of M4 in a star-forming region corresponds to $\sim \mathrm{M} 2$ at an age of 1 Gyr (e.g., Chabrier et al. 2000), comparable to the ages of the stars in the Solar Neighborhood. Among the nearest stars, the majority are M-dwarfs (e.g., Reid \& Gizis 1997; Henry 1998), again highlighting the importance of understanding the environments of low mass stars to develop a comprehensive disk population study.

To obtain a nearly complete census of far-IR emission across the full population of Taurus, we have performed a large-scale survey of low mass stars and brown dwarfs with Herschel, targeting the M4-L0 members - the Taurus Boundary of Stellar/Substellar (TBOSS) Survey - to extend the exploration of disk mass and structure into the substellar regime with a sensitivity that was not possible prior to Herschel. The nearly complete sample for this study is defined in Sect. 2, followed by the new Herschel PACS observations in Sect. 3. The data analysis including the measurement of far-IR flux densities and construction of the SEDs for each target in the full sample is described in Sect. 4. The results for the members of each evolutionary class, a comparison with Spitzer data, the detection rates, and spatially extended and multiple sources are reported in Sect. 5. In Sect. 6, the discussion covers a number of topics including the dependence of the results on spectral type and companions, the SEDs for different types of targets such as transition disks, and a comparison of the Taurus substellar disks with the population in Ophiuchus. Finally, Sect. 7 summarizes the conclusions.

\section{Sample}

The target sample was selected to provide a comprehensive census of far-IR disk emission from Taurus members spanning both sides of the stellar/substellar boundary. The demarcation of the substellar boundary occurs at a spectral type of M6.25 at the age of Taurus (e.g., Luhman et al. 2005), and the spectral type range of the TBOSS sample is M4-L0. From a Spitzer 3.6-24 $\mu \mathrm{m}$ study of the Taurus population (Luhman et al. 2010), 152 M4-L0 members were included, which represented $99 \%$ of the currently known membership, with the remaining $1 \%$ of the known Taurus population comprised of objects with spectral types earlier than M4. Of the newest Taurus members identified in a Spitzer study including $70 \mu \mathrm{m}$ data (Rebull et al. 2010), all but 2 with spectral types of M4 and later were included in the Luhman et al. (2010) study. Of the 154 M4-L0 members listed in the combination of the Spitzer studies, Herschel Photodetector Array Camera and Spectrometer (PACS; Poglitsch et al. 2010) $70 \mu \mathrm{m}$ or $100 \mu \mathrm{m}$ and $160 \mu \mathrm{m}$ flux densities or detection limits are reported for 150 objects.

The large majority of the Herschel observations, covering 134 targets, were performed as part of our program (OT1_patienc_1). PACS photometry for 12 targets were drawn from the literature - 4 targets from Harvey et al. (2012) from the program GT1_pharve01_2, and 8 targets from Howard et al. (2013) from the program KPOT_bdent_1 as part of the Gas Survey of Protoplanetary Systems (GASPS; Dent et al. 2013). Another 4 targets were observed as part of the guaranteed time key program, KPGT_pandre_1, and for these targets PACS photometry measurements were made from the reduced archive maps. For the 4 targets not observed by Herschel, far-IR Spitzer measurements exist for J04251550+2829275, J04355760+2253574, IRAS S04414+2506 (Rebull et al. 2010), but no measurements exist for XEST 26-052. Table 1 reports the 2MASS name, alternate name, evolutionary class and spectral type of each of the 154 M4-L0 Taurus members. The 153-object TBOSS sample consists of the known 154 Taurus members with either Herschel or Spitzer far-IR photometry, and is comprised of 7 Class I, 69 Class II, and 77 Class III members, and 63 of the targets are brown dwarfs (M6.25-L0). The spectral types have been compiled from the original reference (noted in Table 1), and for the targets with more than one spectral type measurement reported, the most recent result was adopted for this study. Figure 1 shows the distribution of the TBOSS sample across the Taurus molecular cloud.

\section{Observations}

Of the 153 targets in the TBOSS sample, 150 were observed with the Herschel PACS instrument. PACS records two wavelengths simultaneously, and the observations of 146 include both $70 \mu \mathrm{m}$ data from the blue channel and $160 \mu \mathrm{m}$ data from the red channel. Amongst the 146 targets, 8 targets were also observed with the PACS blue channel operating at $100 \mu \mathrm{m}$. Observations of the remaining 4 targets of the 150 total were carried out with PACS operating at $100 \mu \mathrm{m}$ and $160 \mu \mathrm{m}$. For majority of the sample, the 134 targets observed under our Herschel PACS program 

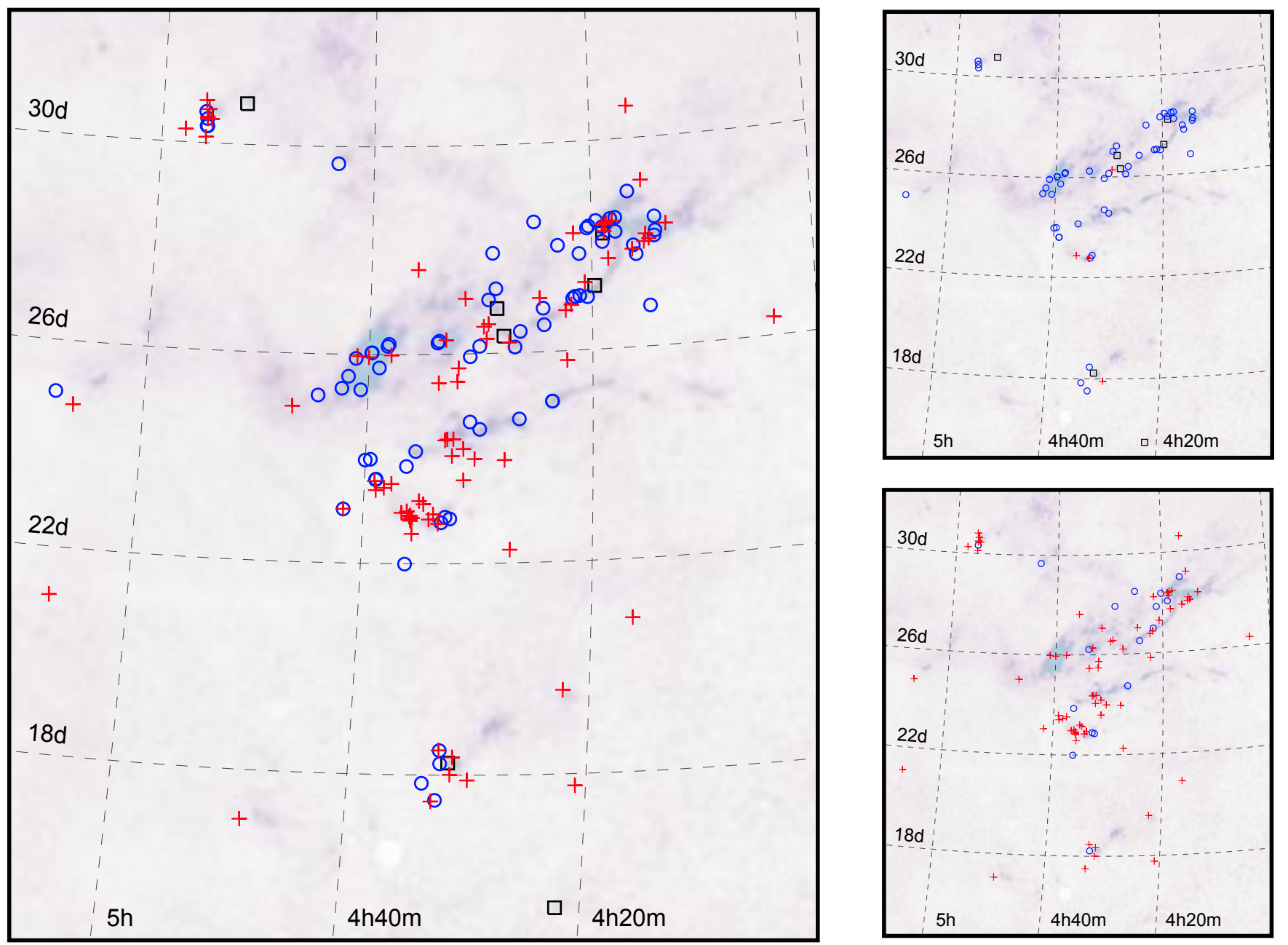

Fig. 1. Left panel: spatial distribution of the TBOSS sample across the Taurus molecular cloud (shown in the map of extinction from Dobashi et al. 2005). Black squares indicate Class I objects, blue circles indicate Class II objects, and red crosses indicate Class III objects. Top right panel: distribution of the TBOSS sample with Herschel PACS detections. Bottom, right panel: distribution of the TBOSS sample with Herschel PACS upper limits.

(OT1_patienc_1), the mapping strategy involved obtaining two scan maps per target, with each map at a different orientation $\left(70^{\circ}\right.$ and $\left.110^{\circ}\right)$. The cross scans are designed to reduce the stripping effect of the $1 / f$ noise and, consequently, to provide more homogeneous and higher redundancy coverage of the map area. The medium mapping scan speed $\left(20^{\prime \prime} / \mathrm{s}\right)$ was employed with scan leg lengths of 3.'0, cross scan steps of 4.'0, and a total of 8 scan legs per orientation. Each map was centered on the target and the field-of-view was typically $3^{\prime} \times 7^{\prime}$. The pointing accuracy of Herschel is $0 .^{\prime} 8^{1}$, and the beam size is $5^{\prime \prime} 6$ in the $70 \mu \mathrm{m}$ maps, 6.' 8 in the $100 \mu \mathrm{m}$ maps and $11^{\prime \prime} 3$ in the $160 \mu \mathrm{m}$ maps. The integration time between the PACS channels is the same, and the total on-source integration time was 180 s for all but 5 targets for which the integration time was $240 \mathrm{~s}$. Table 2 reports the Herschel scan and cross scan ID numbers along with the observation date, observation duration and total on-source integration time for each target in the OT1_patienc_1 program. Table 3 summarizes the program ID, scan ID numbers, observation date, and observation duration per target for the 16 targets not observed in the OT1_patienc_1 program.

\footnotetext{
1 http://herschel.esac.esa.int/twiki/bin/view/Public/ SummaryPointing
}

For the 3 targets of the 153-object TBOSS sample with Spitzer Multiband Imaging Photometer (MIPS; Rieke et al. 2004) measurements only, the observation details are reported in Rebull et al. (2010).

\section{Data analysis}

\subsection{Measurement of PACS flux densities}

The Herschel program OT1_jpatienc_1 data were reduced using the Herschel Interactive Processing Environment (HIPE; Ott 2010) software version 9.0.0. For each target, the scan and corresponding cross scan were pre-processed using the standardized routines given in the PACS Data Reduction Guide (version 7, June 2011), and the calibration employed the PACS calibration file set version 48 . The initial map was used to identify any bright sources that needed to be masked prior to performing the multiresolution median transform (MMT) de-glitching routine and applying a high pass filter to each data set. The location of any point source with a signal stronger than three times the standard deviation of the initial map was masked.

Depending on the brightness and size of the source in the initial map, a different number of frames were used in the high pass filtering, as suggested in the data reduction guide. For the 
Table 3. Observing log for the TBOSS sample observed with Herschel PACS under other programs.

\begin{tabular}{|c|c|c|c|c|c|c|}
\hline 2MASS & Other name & $\begin{array}{c}\text { Scan } \\
\text { ID }\end{array}$ & $\begin{array}{c}\text { Cross scan } \\
\text { ID } \\
\end{array}$ & $\begin{array}{l}\text { UT } \\
\text { date }\end{array}$ & $\begin{array}{c}\text { Total } \\
\text { duration (s) }\end{array}$ & $\begin{array}{l}\text { Program } \\
\text { ID }\end{array}$ \\
\hline \multirow[t]{2}{*}{ J04131414+2819108 } & LkCa 1 & 1342216513 & 1342216514 & 2011 Mar. 21 & 552 & KPOT_bdent_1 \\
\hline & & $(1342216515)$ & (1342216516) & 2011 Mar. 21 & 552 & KPOT_bdent_1 \\
\hline \multirow[t]{2}{*}{ J04150515+2808462 } & CIDA 2 & 1342216529 & 1342216530 & 2011 Mar. 21 & 552 & KPOT_bdent_1 \\
\hline & & $(1342216531)$ & $(1342216532)$ & 2011 Mar. 21 & 552 & KPOT_bdent_1 \\
\hline J04233539+2503026 & FU Tau A & (1342227304) & (1342227305) & 2011 Aug. 24 & 21222 & KPGT_pandre_1 \\
\hline J04233573+2502596 & FU Tau B & (1342227304) & (1342227305) & 2011 Aug. 24 & 21222 & KPGT_pandre_1 \\
\hline J04242646+2649503 & CFHT 9 & 1342227059 & 1342227060 & 2011 Aug. 21 & 2690 & GT1_pharve01_2 \\
\hline \multirow[t]{2}{*}{ J04292071+2633406 } & $\mathrm{J} 1-507$ & 1342227979 & 1342227980 & 2011 Sep. 04 & 552 & KPOT_bdent_1 \\
\hline & & $(1342227981)$ & (1342227982) & 2011 Sep. 04 & 552 & KPOT_bdent_1 \\
\hline \multirow[t]{2}{*}{ J04292971+2616532 } & FW Tau $\mathrm{A}+\mathrm{B}+\mathrm{C}$ & 1342227987 & 1342227988 & 2011 Sep. 04 & 552 & KPOT_bdent_1 \\
\hline & & (1342227989) & (1342227990) & 2011 Sep. 04 & 552 & KPOT_bdent_1 \\
\hline J04300724+2608207 & KPNO 6 & 1342227012 & 1342227013 & 2011 Aug. 21 & 3140 & GT1_pharve01_2 \\
\hline J04305718+2556394 & KPNO 7 & 1342227999 & 1342228000 & 2011 Sep. 04 & 3140 & GT1_pharve01_2 \\
\hline \multirow[t]{2}{*}{ J04312382+2410529 } & V927 Tau A+B & 1342227055 & 1342227056 & 2011 Aug. 21 & 552 & KPOT_bdent_1 \\
\hline & & (1342227057) & (1342227058) & 2011 Aug. 21 & 552 & KPOT_bdent_1 \\
\hline \multirow{2}{*}{$\mathrm{J} 04313613+1813432$} & LkHa 358 & 1342228928 & 1342228929 & 2011 Sep. 19 & 552 & KPOT_bdent_1 \\
\hline & & (1342228930) & $(1342228931)$ & 2011 Sep. 19 & 552 & KPOT_bdent_1 \\
\hline \multirow[t]{2}{*}{ J04315844+2543299 } & $\mathrm{J} 1-665$ & 1342228001 & 1342228002 & 2011 Sep. 04 & 552 & KPOT_bdent_1 \\
\hline & & (1342228003) & (1342228004) & 2011 Sep. 04 & 552 & KPOT_bdent_1 \\
\hline J04321786+2422149 & CFHT 7 & (1342228005) & (1342228006) & 2011 Sep. 04 & 39224 & KPGT_pandre_1 \\
\hline \multirow[t]{2}{*}{ J04323028+1731303 } & GG Tau Ba+Bb & 1342228940 & 1342228941 & 2011 Sep. 19 & 552 & KPOT_bdent_1 \\
\hline & & (1342228942) & (1342228943) & 2011 Sep. 19 & 552 & KPOT_bdent_1 \\
\hline J04330945+2246487 & CFHT 12 & 1342227013 & 1342227014 & 2011 Aug. 21 & 3140 & GT1_pharve01_2 \\
\hline J04353536+2408266 & IRAS $04325+2402 \mathrm{C}$ & (1342228005) & (1342228006) & 2011 Sep. 04 & 39224 & KPGT_pandre_1 \\
\hline
\end{tabular}

Notes. Targets are ordered by RA. The observations listed were performed with the PACS photometer blue1 channel (70 $\mu \mathrm{m})$ and the red channel $(160 \mu \mathrm{m})$. For the scan ID numbers within parenthesis, observations were performed with the PACS photometer blue2 channel (100 $\mu \mathrm{m})$ and the red channel $(160 \mu \mathrm{m})$.

9 brightest and/or resolved targets, a high pass filter radius corresponding to 51 frames in the blue channel and 71 frames in the red channel was used; for the 125 fainter point sources, increments of 31 frames in the blue channel and 51 frames in the red channel were used to filter the thousands of frames involved in the scans of each target. After filtering, each scan and cross-scan was re-processed and the final map for each target was formed by a co-addition using the photProject() task.

The target flux densities were measured using aperture photometry, with the aperture size determined by the observation wavelength and object size. Among the 146 targets observed at $70 \mu \mathrm{m}$ and $160 \mu \mathrm{m}, 141$ are unresolved point sources and 5 are spatially resolved. For the point sources, the aperture radii of $5^{\prime \prime} .5$ at $70 \mu \mathrm{m}$ and 10.5 at $160 \mu \mathrm{m}$ recommended by the Herschel Science Center ${ }^{2}$ were used to measure the target flux densities. For the extended sources, the flux densities were measured within a $3 \sigma$ contour, derived from three times the standard deviation of the map noise. The source flux density error was calculated as the standard deviation of the flux density in nine comparison apertures of the same size as the target aperture and distributed in the high coverage area of each map. Upper limits were determined by three times the standard deviation of the nine comparison apertures. Finally, aperture corrections defined in a Herschel Science Center technical note ${ }^{2}$ were applied to the source flux densities and upper limits. Additional absolute flux calibration uncertainties of $2.6 \%$ at $70 \mu \mathrm{m}$ and $4.2 \%$ at $160 \mu \mathrm{m}$ as reported in the technical note ${ }^{2}$ are not included in the uncertainties reported here. For the 4 targets observed as

\footnotetext{
2 Technical note: PICC-ME-TN-037
}

part of the KPGT_pandre_1 program, 3 targets were unresolved point sources and 1 target was spatially resolved. For the point sources, flux densities were measured from aperture photometry of the level 2.5 processed maps ${ }^{3}$; an aperture of 5'.6 was used for the $100 \mu \mathrm{m}$ data. For the resolved target, the flux density was measured within a $3 \sigma$ contour from the level 2.5 processed map. For the 12 targets observed as part of the GT1_pharve01_2 and KPOT_bdent_1 programs, the flux densities were obtained from the literature (Harvey et al. 2012; Howard et al. 2013).

\subsection{Construction of spectral energy distributions}

Spectral energy distributions were constructed for all targets of the TBOSS sample. The details of the source photometry included in the SEDs from non-Herschel observations are given in this section, and the resulting SED plots are presented and discussed in Sect. 6. In addition to the Herschel PACS photometry, the SEDs include photometry from optical to millimeter wavelengths when available.

The optical photometry measurements consist of $R_{\mathrm{C}}$ and $I_{\mathrm{C}}$ band measurements compiled from the literature. The original measurements were in the $R_{\mathrm{C}}$ and $I_{\mathrm{C}}$ filters for those targets reported in Kenyon \& Hartmann (1995), whilst all other compiled literature studies transformed the measured photometric value into the Cousins system (Guieu et al. 2007, 2006; Luhman 2000, 2004; Luhman et al. 2003a; Briceño et al. 1999, 2002; Martín et al. 2001). For targets with more than one photometric

3 Here level 2.5 maps are the products of calibrated and combined PACS scan and cross-scan data. 
measurement, the median value was adopted. Uncertainties were not reported in up to half of the the literature sources, therefore, to account for both source variability and the uncertainties in the photometric transformation to the Cousins system, adopted common values for the $R_{\mathrm{C}}$ and $I_{\mathrm{C}}$ uncertainties were used (e.g., Briceño et al. 2002; Mayne et al. 2012). The error adopted for $R_{\mathrm{C}}$ was $0.8 \mathrm{mag}$, and the error adopted for $I_{\mathrm{C}}$ was $0.3 \mathrm{mag}$. Magnitudes were converted to flux densities using zero-points of $3080 \mathrm{Jy}$ and $2550 \mathrm{Jy}$ for $R_{\mathrm{C}}$ and $I_{\mathrm{C}}$, respectively (Bessell 1979). The $R_{\mathrm{C}}$ and $I_{\mathrm{C}}$ flux densities, and literature references are listed in Table A.1.

In the near-infrared, $J H K_{\mathrm{S}}$ magnitudes for all targets were extracted from the Two Micron All Sky Survey (2MASS) point source catalog (Skrutskie et al. 2006) and were converted into flux densities using zero-points of $1594 \pm 28 \mathrm{Jy}, 1024 \pm 20 \mathrm{Jy}$ and $667 \pm 13 \mathrm{Jy}$ (Cohen et al. 2003) for $J, H$, and $K_{\mathrm{S}}$, respectively. The $2 \mathrm{MASS} J H K_{\mathrm{S}}$ flux densities and uncertainties are listed in Table A.1.

In the mid-IR, with the Spitzer Infrared Array Camera (IRAC: 3.6, 4.5, 5.8 and $8.0 \mu \mathrm{m}$; Fazio et al. 2004), and at $24 \mu \mathrm{m}$ with the Multiband Imaging Photometer for Spitzer (MIPS; Rieke et al. 2004), magnitudes were compiled from Luhman et al. (2010) and Rebull et al. (2010). The mean-weighted magnitudes were calculated for targets in which multiepoch observations are reported. Magnitudes were converted into flux densities using the zero-points of $281 \pm 4 \mathrm{Jy}, 180 \pm 3 \mathrm{Jy}, 115 \pm 2 \mathrm{Jy}$ and $65 \pm 1 \mathrm{Jy}$ at $3.6,4.5,5.8$ and $8.0 \mu \mathrm{m}$ respectively (Reach et al. 2005), and $7.2 \pm 0.1 \mathrm{Jy}$ at $24 \mu \mathrm{m}$ (Engelbracht et al. 2007). Additionally, the Wide-field Infrared Survey Explorer (WISE) All-Sky Data Release (Cutri \& et al. 2012) was used to extract photometry for all targets measured in the four WISE channels. The WISE magnitudes were converted into flux densities using the zero-points of $310 \pm 5 \mathrm{Jy}, 172 \pm 3 \mathrm{Jy}, 31.7 \pm 0.5 \mathrm{Jy}$ and $8.4 \pm 0.1 \mathrm{Jy}$ at $3.4,4.6,12$ and $22 \mu \mathrm{m}$ respectively (Wright et al. 2010). The IRAC, WISE flux densities and uncertainties are given in Table A.2. The MIPS-1 flux densities and uncertainties are given in Table A.2.

In the far-IR, Spitzer MIPS-2 $(70 \mu \mathrm{m})$ measurements were compiled from Rebull et al. (2010), using the zero-point of $0.78 \pm 0.01 \mathrm{Jy}$ (Gordon et al. 2007). In addition to the new Herschel PACS observations presented here, a subset of the TBOSS sample $70 \mu \mathrm{m}, 100 \mu \mathrm{m}$ and $160 \mu \mathrm{m}$ PACS measurements were compiled from Harvey et al. (2012) and Howard et al. (2013). All far-IR flux densities and uncertainties are given in Table A.2.

The submillimeter and millimeter measurements consist of observations at 350-1200 $\mu \mathrm{m}$, and were compiled from the following studies: Klein et al. (2003); Young et al. (2003); Andrews \& Williams (2005); Bourke et al. (2006); Andrews et al. (2008); Bouy et al. (2008); Francesco et al. (2008); Harris et al. (2012); and Mohanty et al. (2013). At $1.3 \mathrm{~mm}$ and $2.6 \mathrm{~mm}$, measurements were compiled from the following studies: Beckwith et al. (1990); Osterloh \& Beckwith (1995); Motte \& André (2001); Scholz et al. (2006); Schaefer et al. (2009); Phan-Bao et al. (2011); Ricci et al. (2013) and Andrews et al. (2013). All flux densities and uncertainties for the submillimeter and millimeter data are given in Table A.4.

In addition to the observed photometry, the SEDs for each target also show the underlying best-fit stellar atmospheric model. The two parameters that define the model atmosphere are effective stellar temperature $\left(T_{\text {eff }}\right)$ and surface gravity $(\log (g))$; metallicity was assumed to be Solar. The atmospheric model temperature was fixed based on the adopted spectral type of each target and using the temperature-spectral type conversion from Luhman et al. (2003b). The value of $\log (g)$ and the initial value of stellar radius $\left(R_{\text {star }}\right)$ was set from the 1 Myr evolutionary models of Baraffe et al. (1998) for targets with $T_{\text {eff }}>2700 \mathrm{~K}$ $(\leq \mathrm{M} 8)$ and the models from Chabrier et al. (2000) for targets with $T_{\text {eff }}<2700 \mathrm{~K}$. The model atmosphere flux is scaled by $R_{\text {star }}$ and extinction before plotting on the SED. The values of $R_{\text {star }}$ and visual extinction $\left(A_{V}\right)$ were used as free parameters in the fitting process, since the atmospheric models, set by target spectral type, resulted in poor fits for some cases. The initial extinction estimate for each target was scaled to $A_{V}$ from the reported $A_{J}$ or $A_{H}$, following the extinction law of Mathis (1990). For targets with no reported extinction, the initial $A_{V}$ was estimated using $A_{V}=9.44 \times E(J-H)$, derived from the extinction law of Mathis (1990), where $E(J-H)=(J-H)-(J-H)_{0}$ and is the excess with respect to the expected stellar photosphere. The intrinsic $(J-H)_{0}$ colors were taken from Luhman et al. (2010). The visual extinctions compiled from the literature and those derived in this paper are given in Table 4.

The best-fit atmospheric model for each target was obtained by minimizing the $\chi^{2}$ value, summed over the 2MASS $J H K_{\mathrm{S}}$ bands where detections are reported. Varying the value of $A_{V}$ also accounts for the cases in which there is a disk excess affecting the photometry. $A_{V}$ and $R_{\text {star }}$ were typically constrained to vary from no extinction to twice the reported $A_{V}$ values and from 0.4 to 2 times the derived stellar radii values. The reported $A_{V}$ and $R_{\text {star }}$ values for each target, and the corresponding best-fit SED values are listed in Table 4 and are also shown in Fig. 2. The SEDs show the PHOENIX-based (Hauschildt et al. 1999), "BT-settl" models (Allard et al. 2003, 2011) for targets with $T_{\text {eff }}>2700 \mathrm{~K}$ and the "AMES-Dusty" models (Allard et al. $2001)$ for targets with $T_{\text {eff }}<2700 \mathrm{~K}$. The redding law from Mathis (1990) with the optical total-to-selective extinction ratio of $R_{v}=3.1$ was applied to the stellar atmospheric models.

For the multiple systems with angular separations in the range $\sim 5-11^{\prime \prime}-\mathrm{FU}$ Tau $\mathrm{A}+\mathrm{B}$, GG Tau $\mathrm{Ba}+\mathrm{Bb}$, IRAS 04191+1523 A+B, and IRAS 04325+2402 AB+C (Luhman et al. 2009b; Duchêne et al. 2004) - emission longward of the mid-IR is unresolved. For these systems, the combined system photometry were compiled, and the underlying best-fit spectra displayed on the SEDs was combined from the spectral types of both the primary and secondary components. The best-fit extinctions and stellar radii for these four multiples systems are given in Table 4. Furthermore, for the specific case of $\mathrm{GG}$ Tau $\mathrm{Ba}+\mathrm{Bb}$, with an angular separation of 10 .'75 from the primary system $\mathrm{Aa}+\mathrm{Ab}$ (Kraus \& Hillenbrand 2009b), emission is resolved with both Herschel PACS at $70 \mu \mathrm{m}$ (Howard et al. 2013) and the SMA at $1.3 \mathrm{~mm}$ (Harris et al. 2012). The SED for GG Tau $\mathrm{Ba}+\mathrm{Bb}$ was constructed with photometry from the secondary system, and the underlying best-fit spectrum was combined from both the spectral types of the $\mathrm{Ba}$ and $\mathrm{Bb}$ components.

\section{Results}

Of the 150 TBOSS targets observed with Herschel, 58 were detected, 2 have unresolved emission from earlier spectral type companions, and 90 have upper limits. The PACS photometry compilation for these 150 targets is reported in Table 5. The spatial distribution of the full TBOSS sample is shown in Fig. 1, along with the corresponding subset of detections and upper limits. The Herschel results for each evolutionary class are reported in Sect. 5.1 (Class I), 5.2 (Class II), and 5.3 (Class III). After the summary of Herschel results, the PACS flux densities are compared with Spitzer MIPS flux densities in 

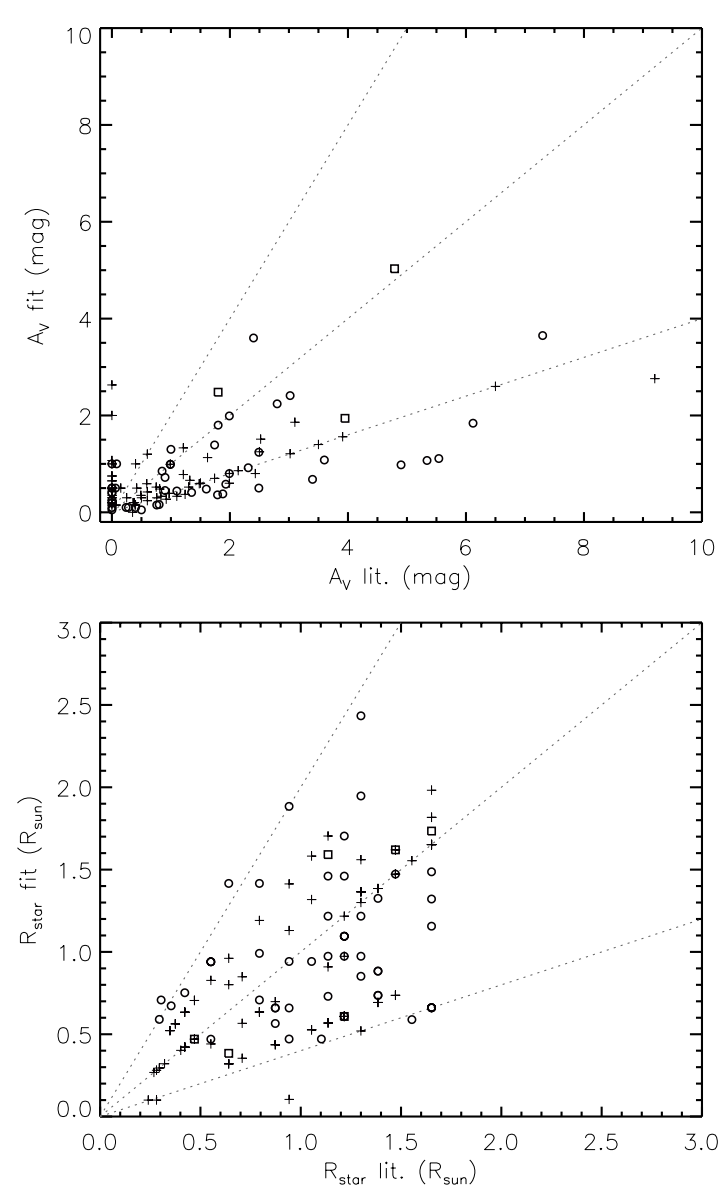

Fig. 2. Comparison plots of the SED best-fit values of $A_{V}$ (top) and $R_{\text {star }}$ (bottom) against those reported in the literature. Literature $A_{V}$ values are compiled from the references listed in Table $4 . R_{\text {star }}$ values are derived from the spectral types reported in the literature, using the temperature conversion of Luhman et al. (2003b) and the 1 Myr evolutionary models of Baraffe et al. (1998) and Chabrier et al. (2000). Squares indicate Class I objects, circles indicate Class II objects, and crosses indicate Class III objects. The dotted lines represent fits that are $0.4,1$ and 2 times that of the literature values.

Sect. 5.4. Combining the Herschel and Spitzer results, the overall 153-object TBOSS sample far-IR detection rates are calculated in Sect. 5.5. Although the Herschel beam size corresponds to $>750 \mathrm{AU}$ in Taurus, several TBOSS targets are spatially extended, and these cases are reported in Sect. 5.6.

\subsection{Class I population}

All of the seven Class I targets within the TBOSS sample are detected in both PACS blue $(70 \mu \mathrm{m}$ or $100 \mu \mathrm{m})$ and red $(160 \mu \mathrm{m})$ channels. The Class I targets include the brightest targets amongst the TBOSS sample observed with Herschel, although the two brightest Class I sources - IRAS 04191+1523 B and IRAS $04248+2612$ - are unresolved binaries, as noted in Table 5. The flux densities of the isolated Class I substellar objects range from $\sim 270 \mathrm{mJy}$ to $>4 \mathrm{Jy}$, larger than nearly all of the Class II sources and indicate that a substantial amount of dust is present around brown dwarfs in the early stage of evolution. The maps for the spatially extended Class I targets are given in Sect. 5.6 and the maps of the remaining unresolved Class I targets are given in Appendix B.

\subsection{Class II population}

The majority ( $>70 \%$ ) of the Class II TBOSS targets are detected with the PACS blue channel at either $70 \mu \mathrm{m}$ or $100 \mu \mathrm{m}$, with a large range of flux densities spanning three orders of magnitude. Approximately half of the Class II TBOSS sample also have PACS $160 \mu \mathrm{m}$ flux densities. Furthermore, several of the detected Class II targets have flux densities comparable to the Class I targets. Table 5 reports the flux densities and upper limits. The angular resolution degrades at longer wavelengths, and the source $\mathrm{JH} 112 \mathrm{~B}$, is undetected at $70 \mu \mathrm{m}$, but a long wavelength detection is due to contamination from emission associated with an earlier spectral type primary. Additionally, the $100 \mu \mathrm{m}$ emission witnessed for IRAS $04325+2402 \mathrm{C}$ (shown in Fig. 5) is unresolved emission from an earlier spectral type primary, and is further discussed in Sect. 5.6. The maps of the detected Class II sources are given in Appendix B.

\subsection{Class III population}

Only a small subset $(<5 \%)$ of Class III TBOSS targets are detected at one or more wavelengths in the Herschel data. Although the overall frequency of detected disks is low for Class III targets, the measured flux densities of the few Class III detections are larger than some Class II targets of equivalent spectral type. One of the detected Class III targets - XEST 08-033 - is unusual, since it is the only source with a non-detection at $70 \mu \mathrm{m}$ and a longer wavelength detection uncontaminated by a more massive primary. The $160 \mu \mathrm{m}$ map for XEST 08-033 shows two sources unlike most of the detected targets. The source at the coordinates of XEST 08-033 is unresolved as expected for a circumstellar disk. Maps of the one detected Class III target in which the emission is spatially extended - XEST $17-036$ - is given in Sect. 5.6, and the maps of the remaining detected Class III targets are given in Appendix B.

\subsection{Comparison with Spitzer MIPS}

Among the TBOSS targets detected at $70 \mu \mathrm{m}$ with the Herschel PACS maps, 21 sources have existing $70 \mu \mathrm{m}$ detections with Spitzer MIPS data (Rebull et al. 2010). A plot of the PACS $70 \mu \mathrm{m}$ flux densities as a function of the corresponding MIPS $70 \mu \mathrm{m}$ flux densities for these 21 sources are shown in Fig. 3. For the sources with flux densities $<1 \mathrm{Jy}$, the agreement between the two values is typically within the MIPS absolute flux calibration uncertainty $(<10 \%)$. For the six sources brighter than $1 \mathrm{Jy}$, the discrepancy between the PACS and MIPS fluxes range from 25-40\%, and the Herschel PACS flux is systematically higher. As noted in a Herschel Science Center technical note ${ }^{4}$, the processed PACS data are affected by the high pass filter width, source masking, drizzling, and aperture sizes used in the photometry. The contribution of these factors is expected to have an impact on the final flux densities by only a small percent and cannot explain the full discrepancy between the larger systematic offset. The most likely cause is due to the impact of nonlinearity for MIPS data compared to PACS; the PACS detector is linear up to flux density levels well above the brightest TBOSS target, while the MIPS detector is non-linear for flux densities above $\sim 1 \mathrm{Jy}^{5}$.

\footnotetext{
4 Technical note: PICC-ME-TN-037.

5 https://nhscsci.ipac.caltech.edu/sc/index.php/Pacs/ AbsoluteCalibration
} 


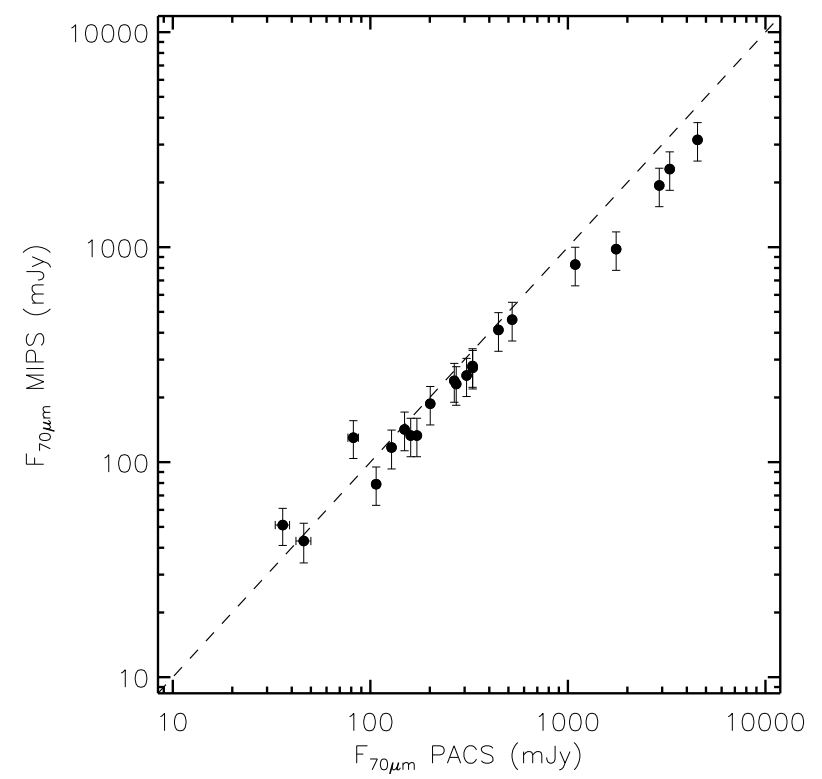

Fig. 3. Relation of PACS $70 \mu \mathrm{m}$ and corresponding MIPS $70 \mu \mathrm{m}$ flux densities (Rebull et al. 2010) for the TBOSS targets detected with both instruments. Non-linearity of the MIPS detector at $\sim 1 \mathrm{Jy}$ is the likely cause of the $25-40 \%$ flux discrepancy seen for the targets $>1 \mathrm{Jy}$, measured with PACS.

\subsection{Far-IR detection rates for Taurus members}

Histograms of the TBOSS sample observed with Herschel PACS are plotted in Fig. 4 with detections indicated. By combining results from the TBOSS far-IR observations with similar Herschel PACS (Howard et al. 2013) and Spitzer MIPS (Rebull et al. 2010) measurements of the earlier spectral type Taurus members, a comprehensive accounting of the detection rates above and below the stellar/substellar boundary is calculated and given in Table 6. The detections rates at $70 \mu \mathrm{m}$ of Class I and Class II targets of any spectral type are $93 \%$ and $84 \%$, respectively. $73 \%$ of Class I targets were detected at both $70 \mu \mathrm{m}$ and $160 \mu \mathrm{m}$, and $52 \%$ of observed Class II targets were similarly detected at both wavelengths. The detection rate declines for later spectral types, however even the substellar (M6.25-L0) Class II members exhibit disk emission at $70 \mu \mathrm{m}$ in the majority $(>50 \%)$ of systems. For the Class III members detected at $70 \mu \mathrm{m}$, and both $70 \mu \mathrm{m}$ and $160 \mu \mathrm{m}$, the detection rate is systematically low ranging from $\sim 5 \%$ for K0-M6 stars to $0 \%$ for M6.25-L0 brown dwarfs. Direct comparisons of the far-IR emission between the substellar members of Taurus and other star-forming regions are not possible, since the TBOSS survey represents a uniquely sensitive and complete study.

\subsection{Spatially extended targets}

Although the Herschel PACS $70 \mu \mathrm{m}$ and $160 \mu \mathrm{m}$ beam sizes are 5'.6 and 11". 3 - which translate into spatial scales of $>750 \mathrm{AU}$ at the distance of Taurus - five TBOSS targets exhibit extended structure in the PACS maps shown in Fig. 5. Amongst the 5 spatially extended targets 3 have been imaged for companions IRAS 04191+1523, IRAS 04248+2612, and IRAS 04325+2402. These three resolved sources are binary systems for the which the angular separations are listed in Table 7 (Duchêne et al. 2004). The components for two of these systems are separated by an angle larger than the $70 \mu$ m beam size. IRAS $04191+1523$ is extended along the binary axis and two peaks are visible in the map. IRAS $04325+2402$ also extended along the binary axis and only one peak is present in the map. In these two wider systems, the TBOSS target is the secondary component. For the remaining resolved binary IRAS $04248+2612$, both components are M4+, only one peak is present, and a large extension to the northwest direction $\sim 45^{\circ}$ to the binary axis is evident. The two additional spatially extended source are the Class I target L1521F-IRS and the Class III target XEST 17-036. In both systems, the emission is largely symmetric. The Class I emission may result from an envelope of dust, however the origin of extended emission in the Class III source is difficult to explain.

Within the $70 \mu \mathrm{m}$ map of the faintly detected brown dwarf J04141188+2811535 is the earlier spectral type object V773 Tau which also appears to be spatially extended, as shown in Fig. 6. The FWHM of V773 Tau is 6.'5, while the FWHM of ten targets with similar brightness is $5^{\prime \prime} .5 \pm 0$ '! 2 . Follow-up interferometric imaging should be able to resolve the disk structure and determine which component or components harbor the disk. In addition to the wide separation TBOSS brown dwarf companion to V773 Tau, the primary is a multiple system that includes many components, within an $0 . ' 3$ radius, there are at least four stars (Boden et al. 2007, 2012). The spatially extended Herschel emission indicates that there may be a circumbinary distribution to the dust.

\section{Discussion}

\subsection{Far-IR emission as a function of spectral type}

The large number of PACS $70 \mu \mathrm{m}$ detections in the TBOSS Class II sample defines the upper boundary of far-IR emission. Connecting the TBOSS population flux densities with previous measurements of earlier spectral type Class II Taurus members reported in the literature (Howard et al. 2013; Rebull et al. 2010) provides the first nearly complete census of $70 \mu \mathrm{m}$ emission for a full population of a star-forming region. Figure 7 plots the $70 \mu \mathrm{m}$ flux densities and upper limits as a function of spectral type spanning K0-M9 for 159 Taurus Class II members, representing $89 \%$ of all objects of this evolutionary class. Although the Spitzer and Herschel GASPS programs targeting earlier spectral types had shallower detection limits than the TBOSS observations, the detection rates for the early spectral types is nearly $100 \%$ as shown in Fig. 8, indicating that the trends seen in the flux density as a function of spectral type are not due to an observational bias. As shown in Fig. 8, the Class II disk frequency based on a $70 \mu \mathrm{m}$ flux density detection declines moderately from nearly $100 \%$ for the earliest spectral types (K0-K7) to $\sim 80 \%$ to the latest stellar spectral types (M4-M6). Below the stellar limit (spectral types M6.25-M9), the frequency of Class II disks drops significantly to $\sim 50 \%$.

The overall shape of the flux density distribution in Fig. 7 exhibits a systematic decline with later spectral types, and the slope of the decline is shallower for K- and early-M stars than for mid- to late-M dwarfs. For comparison with the disk flux density trend, the stellar bolometric luminosity predicted from the 1 Myr evolutionary models of Baraffe et al. (1998), that span the spectral types K7 to $\sim$ M8 (i.e., $T_{\text {eff }} \sim 4100-$ $2300 \mathrm{~K}$ ), is displayed in the lower panel of Fig. 7. For the TBOSS Class II objects detected at $70 \mu \mathrm{m}$, bolometric luminosities were calculated from the best-fit stellar radii derived in the SED fitting procedure described in Sect. 4.2. For the Taurus Class II population outside the TBOSS sample $(<\mathrm{M} 4)$, bolometric luminosities were supplemented from those reported in 

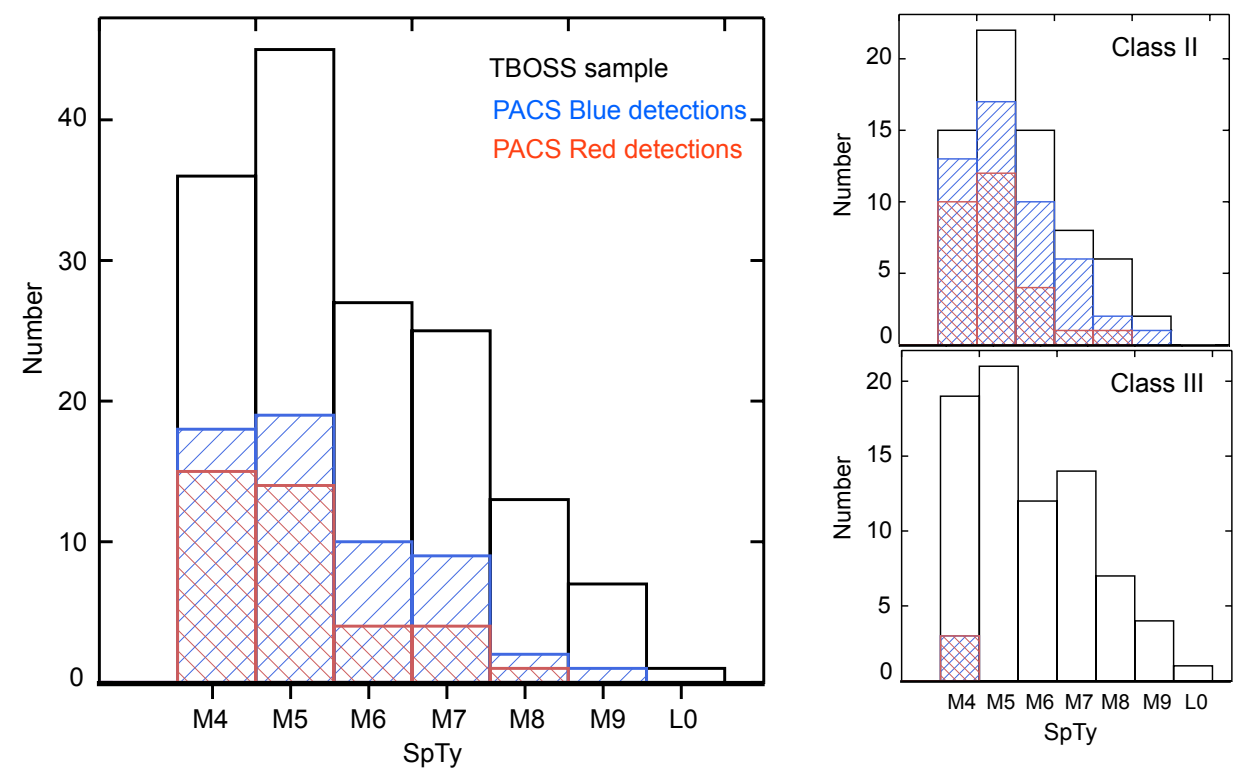

Fig. 4. Histogram of spectral type for the TBOSS sample observed with Herschel PACS. The full observed sample and the number of PACS blue channel $(70 / 100 \mu \mathrm{m})$ detections and red channel $(160 \mu \mathrm{m})$ detections are shown on the left. The Class II and Class III observed sample, and PACS blue and red detections are shown in the top and bottom plots on the right respectively.

Table 6. Detection rates.

\begin{tabular}{lccccc}
\hline \hline Class/ & \multirow{2}{*}{ Total } & Observed & Detection rate & Observed & Detection rate \\
\hline SpTy & 33 & 27 & $93_{-8}^{+2} \%(25)$ & 22 & $73_{-11}^{+7} \%(16)$ \\
\hline Class I & 18 & 13 & $100_{-12}^{+0} \%(13)$ & 10 & $80_{-17}^{+7} \%(8)$ \\
M0-K7 & 8 & 8 & $75_{-20}^{+9} \%(6)$ & 6 & $33_{-13}^{+22} \%(2)$ \\
M4-M6 & 4 & 4 & $100_{-31}^{+0} \%(4)$ & 4 & $100_{-31}^{+0} \%(4)$ \\
M6.25-L0 & 3 & 2 & $100_{-46}^{+0} \%(2)$ & 2 & $100_{-46}^{+0} \%(2)$ \\
\hline Class II & 178 & 156 & $84_{-3}^{+3} \%(131)$ & 138 & $52_{-4}^{+4} \%(72)$ \\
\hline K0-K7 & 37 & 32 & $97_{-7}^{+1} \%(31)$ & 26 & $81_{-10}^{+5} \%(21)$ \\
M0-M3 & 72 & 60 & $87_{-6}^{+3} \%(52)$ & 48 & $50_{-7}^{+7} \%(24)$ \\
M4-M6 & 44 & 43 & $84_{-7}^{+4} \%(36)$ & 41 & $63_{-8}^{+7} \%(26)$ \\
M6.25-L0 & 25 & 21 & $57_{-10}^{+11} \%(12)$ & 23 & $4_{-1}^{+9} \%(1)$ \\
\hline Class III & 128 & 117 & $4_{-1}^{+3} \%(5)$ & 114 & $4_{-1}^{+3} \%(5)$ \\
\hline K0-K7 & 24 & 22 & $5_{-1}^{+9} \%(1)$ & 20 & $5_{-2}^{+10} \%(1)$ \\
M0-M3 & 26 & 20 & $5_{-2}^{+10} \%(1)$ & 18 & $6_{-2}^{+10} \%(1)$ \\
M4-M6 & 43 & 41 & $7_{-2}^{+6} \%(3)$ & 41 & $7_{-2}^{+6} \%(3)$ \\
M6.25-L0 & 35 & 34 & $0_{-0}^{+5} \%(0)$ & 35 & $0_{-0}^{+5} \%(0)$ \\
\hline
\end{tabular}

Notes. The detection rates for the K0-K7, and M0-M3 bins have been tabulated from the Spitzer MIPS observations reported in Rebull et al. (2010) and the Herschel PACS observations reported in Howard et al. (2013). The detection rates for the M4-M6, and M6.25-L0 bins have been tabulated from the Herschel PACS $70 \mu \mathrm{m}$ and $160 \mu \mathrm{m}$ observations reported here and are supplemented with results from the literature (Harvey et al. 2012; Howard et al. 2013).

Andrews et al. (2013). The similarity in the shapes of the stellar bolometric luminosity function and the disk flux density data suggests that the excess flux density fraction from the disk is similar across the full population of Taurus. For the TBOSS sample, the disk excess fraction is plotted as a function of spectral type in Fig. 9. There is no distinction across the stellar/substellar boundary and a Kolmogorov-Smirnov (K-S) test indicates that the stellar and substellar TBOSS samples are drawn from the same parent distribution.
At any given spectral type, there is a significant spread in the far-IR emission, typically over an order of magnitude, as seen in Fig. 7. To investigate possible origins of the large dispersion in disk flux density, we generated a series of model SEDs with the radiative transfer code MCFOST (Pinte et al. 2006, 2009) and considered the range of $70 \mu \mathrm{m}$ flux densities in the model SEDs. Rather than creating an exhaustive multi-parameter grid search on any given target, we varied individual disk parameters about a reference disk defined by the following parameters: inner 
Table 7. Multiplicity of the different categories of disk type objects identified within TBOSS sample.

\begin{tabular}{|c|c|c|c|c|c|c|c|}
\hline $\begin{array}{l}\text { 2MASS } \\
\text { (1) }\end{array}$ & $\begin{array}{l}\text { Other } \\
\text { name } \\
(2)\end{array}$ & $\begin{array}{c}\text { Class } \\
(3)\end{array}$ & $\begin{array}{l}\text { SpTy. } \\
\text { (4) }\end{array}$ & $\begin{array}{l}\text { Companion } \\
\text { name } \\
\text { (5) }\end{array}$ & $\begin{array}{l}\rho \\
\left({ }^{\prime \prime}\right) \\
(6)\end{array}$ & $\begin{array}{l}\text { PACS } \\
\text { Blue det. } \\
(7)\end{array}$ & $\begin{array}{r}\text { Ref. } \\
\text { (8) }\end{array}$ \\
\hline \multicolumn{8}{|c|}{ Extended disk objects } \\
\hline J04220007+1530248 & IRAS $04191+1523 \mathrm{~B}$ & I & M6-M8 & IRAS $04191+1523 \mathrm{~A}$ & 6.09 & $\mathrm{y}$-contam ${ }^{\star}$ & 5 \\
\hline J04275730+2619183 & IRAS 04248+2612 & $\mathrm{I}$ & M4.5 & IRAS $04248+2612 \mathrm{~B}$ & 4.55 & $y$-contam & 5 \\
\hline J04353536+2408266 & IRAS $04325+2402 \mathrm{C}$ & II & M6-M8 & IRAS $04325+2402 \mathrm{AB}^{a}$ & 8.15 & $\mathrm{y}$-contam ${ }^{\star}$ & 5 \\
\hline \multicolumn{8}{|c|}{ Transition disk objects } \\
\hline J04144730+2646264 & FP Tau & II & M4 & $\ldots$ & $\ldots$ & $\mathrm{y}$ & 9 \\
\hline J04161210+2756385 & $\ldots$ & II & M4.75 & $\ldots$ & $\ldots$ & $\mathrm{y}$ & 6 \\
\hline J04190110+2819420 & V410 X-ray 6 & II & M4.5 & $\ldots$ & $\ldots$ & $\mathrm{y}$ & 4 \\
\hline J04202555+2700355 & $\ldots$ & II & M5.25 & $\ldots$ & $\ldots$ & $\mathrm{y}$ & 9 \\
\hline J04210934+2750368 & $\ldots$ & II & M5.25 & J04210934+2750368 B & 0.77 & $\mathrm{n}$ & 4 \\
\hline J04213459+2701388 & $\ldots$ & II & M5.5 & $\ldots$ & $\ldots$ & $\mathrm{y}$ & 6 \\
\hline J04284263+2714039 & $\ldots$ & II & M5.25 & & 0.62 & $\mathrm{y}$ & 6 \\
\hline J04292971+2616532 & $\mathrm{FW}$ Tau $\mathrm{A}+\mathrm{B}+\mathrm{C}$ & III & M4 & FW Tau A-B & 0.08 & $y$-contam & 13 \\
\hline & & & & FW Tau A-C & 2.36 & $y$-contam & 11 \\
\hline J04322210+1827426 & MHO 6 & II & M4.75 & $\ldots$ & $\ldots$ & $\mathrm{y}$ & 9 \\
\hline J04403979+2519061 & $\ldots$ & II & M5.25 & J04403979+2519061 B & 0.04 & $\mathrm{y}$ & 6 \\
\hline \multicolumn{8}{|c|}{ Truncated disk objects } \\
\hline J04141188+2811535 & $\ldots$ & II & M6.25 & V773 Tau & 26.21 & $\mathrm{y}$ & 8 \\
\hline & & & & V773 Tau A(ab) & $\mathrm{SB}^{b}$ & $y$-contam ${ }^{\star}$ & 2 \\
\hline & & & & V773 Tau A-B & 0.12 & $\mathrm{y}$-contam ${ }^{\star}$ & 3 \\
\hline & & & & V773 Tau A-C & 0.26 & $\mathrm{y}$-contam ${ }^{\star}$ & 3 \\
\hline J04174965+2829362 & V410 X-ray 1 & II & M4 & $\ldots$ & $\ldots$ & $\mathrm{y}$ & 9 \\
\hline J04265732+2606284 & KPNO 13 & II & M5 & $\ldots$ & $\ldots$ & $\mathrm{y}$ & 9 \\
\hline J04321606+1812464 & MHO 5 & II & M7 & $\ldots$ & $\ldots$ & $\mathrm{n}$ & 7 \\
\hline $\mathrm{J} 04432023+2940060$ & CIDA 14 & II & M5 & J04432023+2940060 B & $8.4^{c}$ & $\mathrm{n}$ & 1 \\
\hline \multicolumn{8}{|c|}{ Mixed pair systems ${ }^{d}$} \\
\hline J04414565+2301580 & $\ldots$ & III & M4.5 & $\mathrm{J} 04414565+2301580 \mathrm{Aa}-\mathrm{Ab}$ & 0.23 & $\mathrm{n}$ & 10 \\
\hline & & & & $\mathrm{J} 04414489+2301513$ & 12.81 & $\mathrm{y}$ & 8 \\
\hline & & & & $\mathrm{J} 04414489+2301513 \mathrm{Ba}-\mathrm{Bb}$ & 0.10 & $\mathrm{y}$ & 12 \\
\hline J04554757+3028077 & $\ldots$ & III & M4.75 & $\mathrm{J} 04554801+3028050$ & 6.31 & $\mathrm{n}$ & 8 \\
\hline
\end{tabular}

Notes. Column (1) 2MASS identifier. Column (2) Other name. Column (3) Spectral evolutionary class. Column (4) Spectral type. Column (5) Companion name. Column (6) Projected companion separations. Column (7) Targets with PACS blue channel (70 $\mu \mathrm{m}$ or $100 \mu \mathrm{m})$ detections are designated " $y$ ", and targets with upper limits are designated "n". "y-contam" indicates detected emission that is contaminated due the companion listed, and "y-contam ${ }^{\star}$ " indicates contamination due to the listed companion that is of a spectral type earlier than that of the TBOSS sample $(<\mathrm{M} 4)$. Column (8) Reference of companion separation. ${ }^{(a)}$ IRAS $04325+2402 \mathrm{AB}$ is a speculated binary (Hartmann et al. 1999). ${ }^{(b)}$ Spectroscopic binary (SB). ${ }^{(c)} H S T$ WFC3 archive data was inspected for candidate companions. ${ }^{(d)}$ For the mixed pair systems, the primary component name and properties are listed in Cols. 1-4.

References. (1) This work; (2) Boden et al. (2007); (3) Boden et al. (2012); (4) Cieza et al. (2012); (5) Duchêne et al. (2004); (6) Konopacky et al. (2007); (7) Kraus et al. (2006); (8) Kraus \& Hillenbrand (2009b); (9) Kraus \& Hillenbrand (2009a); (10) Kraus et al. (2011); (11) Kraus et al. (2014); (12) Todorov et al. (2014); (13) White \& Ghez (2001).

and outer radius $\left(R_{\text {in }}, R_{\text {out }}\right)$, minimum and maximum grain size $\left(a_{\min }, a_{\max }\right)$, surface density exponent $(p)$, scale height $\left(H_{0}\right)$, flaring exponent $(\beta)$ and disk mass $\left(M_{\text {disk }}\right)$. As shown in Fig. 10, disk properties such as $R_{\mathrm{in}}, R_{\mathrm{out}}, a_{\mathrm{min}}, a_{\mathrm{max}}, p$, and $M_{\text {disk }}$ do not have a large impact on the $70 \mu \mathrm{m}$ flux density relative to other wavelength regimes, and the PACS data do not constrain these parameters. In contrast, variations in geometrical and structural factors such as disk inclination, $H_{0}$ (equivalent to disk opening angle), and $\beta$, can individually account for variations up to nearly an order of magnitude. Following on from this assessment, properties for a canonical disk model were used to generate a grid of model SEDs, across spectral types ranging from K0-M9, in order to investigate the effect that disk inclination alone can have in the spread of $70 \mu \mathrm{m}$ flux density for objects with the same spectral type. The input stellar properties $\left(T_{\mathrm{eff}}, R_{\mathrm{star}}\right.$, and $\left.M_{\mathrm{star}}\right)$ were set based on the 1 Myr evolutionary models of Palla \& Stahler (1999) for spectral types $<$ K7, Baraffe et al. (1998) for spectral types K7-M8, and those from Chabrier et al. (2000) for spectral types $\geq M 8$. Six of the eight input disk parameters were fixed in accordance to typical values reported in the literature; $a_{\min }=0.03 \mu \mathrm{m}, a_{\max }=1000 \mu \mathrm{m}, H_{0}=10 \mathrm{AU}$ at a reference radius of $100 \mathrm{AU}, \beta=1.125, p=-1.0$ and $R_{\text {out }}=100 \mathrm{AU}$. The two remaining disk parameters were scaled in accordance to the properties of the central object; $M_{\text {disk }}$ was set at $1 \%$ of the stellar mass (e.g. Scholz et al. 2006; Andrews et al. 2013) with a standard gas to dust ratio of $100: 1$, and $R_{\text {in }}$ was set at the sublimation radius for dust grains at $T_{\text {sub }}=1500 \mathrm{~K}$. The SED models were generated for disk inclinations at; face-on $\left(0^{\circ}\right), 60^{\circ}$, and edgeon $\left(90^{\circ}\right)$, from which the $70 \mu \mathrm{m}$ flux densities were extracted and are shown in Fig. 7. Between the face- and edge-on disk 

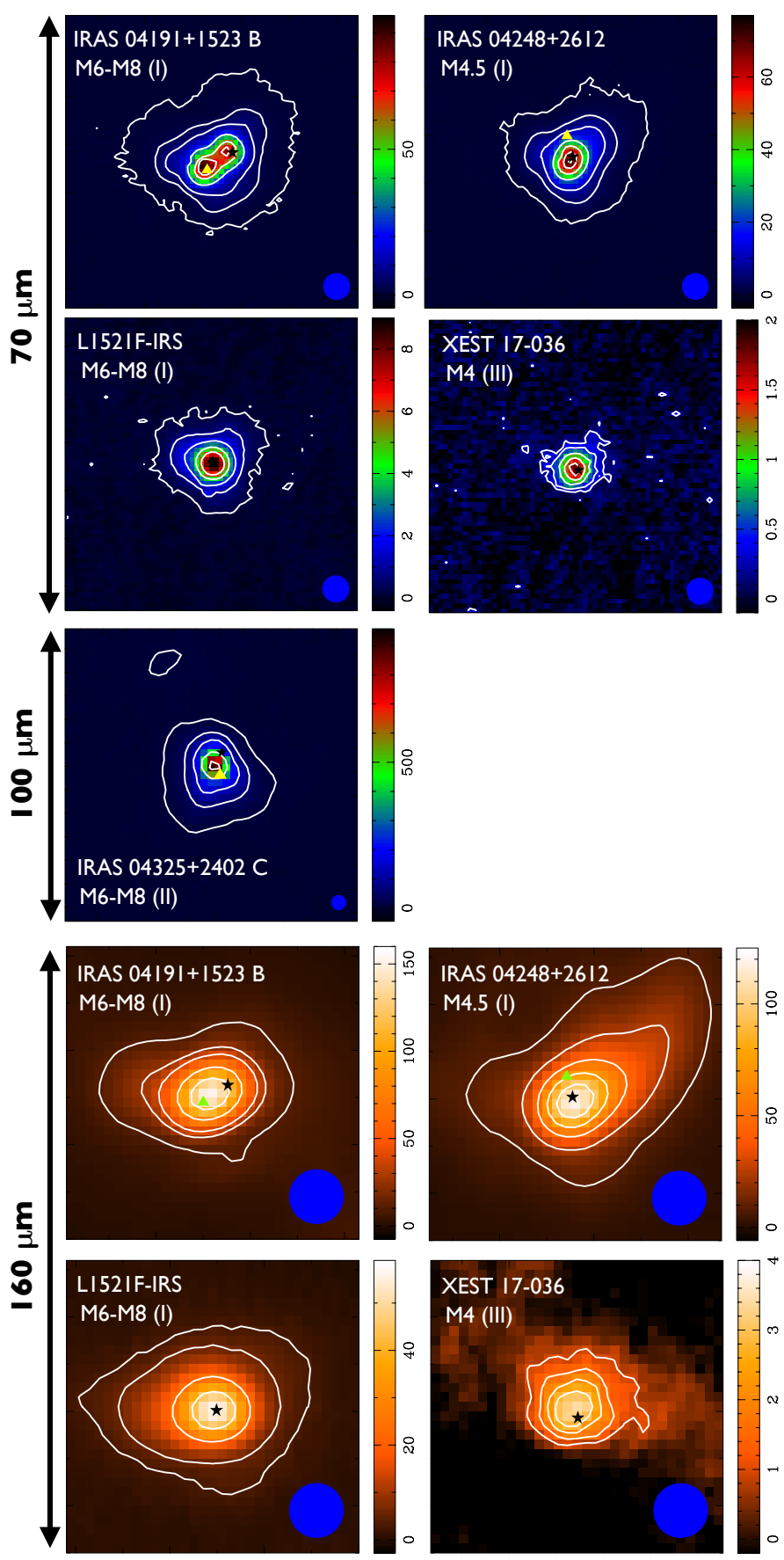

Fig. 5. PACS blue channel $(70 \mu \mathrm{m}$ and $100 \mu \mathrm{m})$ and red channel $(160 \mu \mathrm{m})$ maps of the five spatially extended TBOSS targets. Target name, spectral type and evolutionary class are labeled in the top, lefthand corner of each map. The $70 \mu \mathrm{m}$ and $160 \mu \mathrm{m}$ maps are $60^{\prime \prime}$ in size along each axis, and the $100 \mu \mathrm{m}$ maps are $120^{\prime \prime}$ in size along each axis. Beam sizes are represented by the blue circles in the bottom, right-hand corner of each map. The scale bar shows the intensity of each map in units of mJy/pixel. 2MASS coordinates of the TBOSS targets are represented by the black stars. For binary systems, the position of the companion star are indicted with yellow triangles in the $70 \mu \mathrm{m}$ and $100 \mu \mathrm{m}$ maps, and by green triangles in the $160 \mu \mathrm{m}$ maps. Contours levels begin at $3 \sigma$ in all maps and extend up to $500 \sigma, 400 \sigma, 150 \sigma, 40 \sigma$ and $300 \sigma$ in the blue channel maps of IRAS $04191+1523$ B, IRAS $04248+2612$, L1521F-IRS, XEST $17-036$ and IRAS $04325+2402 \mathrm{C}$ respectively, and up to $50 \sigma$, $60 \sigma, 70 \sigma$ and $10 \sigma$ in the red channel maps of IRAS $04191+1523 \mathrm{~B}$, IRAS 04248+2612, L1521F-IRS and XEST 17-036 respectively.

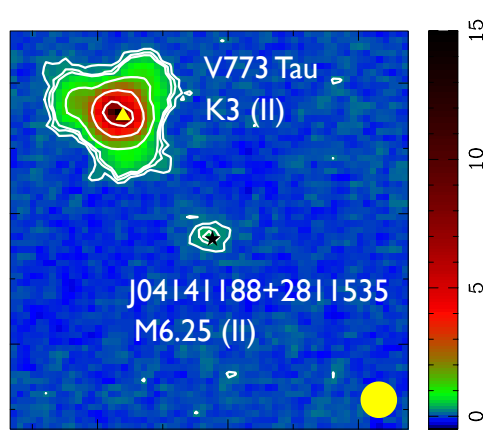

Fig. 6. PACS blue channel $(70 \mu \mathrm{m})$ map of the faintly detected brown dwarf J04141188+2811535 (black star). The earlier type companion V773 Tau (yellow triangle) is also seen within the map and is spatially extended. Target name, spectral type and evolutionary class for each component of the system are indicated on the map. The map is $60^{\prime \prime}$ in size along each axis. The beam size is represented by the yellow circle in the bottom, right-hand corner. Contour levels are plotted at 3, 5, 7, 20, 50 and $100 \sigma$. The scale bar shows the intensity of the map in mJy/pixel.

inclinations, over an order of magnitude spread in flux density is seen across the majority of the spectral range, and $73 \%$ of the observed population is bounded between these two models. Direct observations of disks around brown dwarfs, and constraints from SED modeling results reported in the literature, suggest that the outer disk radius is typically in the range $\sim 10-40 \mathrm{AU}$ for these low mass objects (e.g., Scholz et al. 2006; Luhman et al. 2007; Ricci et al. 2013). In order to ensure that the canonical value $R_{\text {out }}=100 \mathrm{AU}$ does not bias the model results for objects in the brown dwarf regime, a second grid of SEDs were generated in which $R_{\text {out }}$ was scaled as $M_{\text {star }}^{1 / 2}$ (keeping the density across the area of the disk constant across the spectral range) and bound at 10 AU for an object of spectral type M9. No significant change in the $70 \mu$ m flux density was seen between the these two grid of models. Whilst the disk model $70 \mu \mathrm{m}$ flux densities from faceto edge-on exhibit a spread of over a magnitude, and encompass the majority of the observed population, an equivalent trend and spread is seen in the observed bolometric luminosities in the lower panel of Fig. 7. In addition to the $1 \mathrm{Myr}$ isochrone shown in Fig. 7, the isochrones for 0.4 and 2 times that of the model stellar radii are also displayed, and selected based on the results of the SED fitting of the TBOSS sample. To distinguish whether or not the stellar properties alone are the underlying cause of the observed trend and spread in disk flux densities, the TBOSS data points in Fig. 7 are plotted with a size corresponding to the best-fit stellar radii. The random scatter of large $(>1.5$ times the model radii) and small $(<0.5$ times the model radii) best-fit model radii throughout the population indicates that the spread seen in the $70 \mu \mathrm{m}$ disk excess cannot be solely explained due to the range of fluxes of the central source. This further reinforces the requirement for detailed modeling of the disk properties across the full population, and is the subject of a forthcoming paper.

\subsection{Spectral energy distributions of the TBOSS sample}

Spectral energy distributions were constructed for each target of the TBOSS sample by combining the new Herschel far-IR photometry with literature photometry measurements from optical submm/mm wavelengths. The underlying stellar photosphere is also plotted on each SED. For 65 of the 148 TBOSS targets, the Herschel data represents the longest wavelength measurement. 


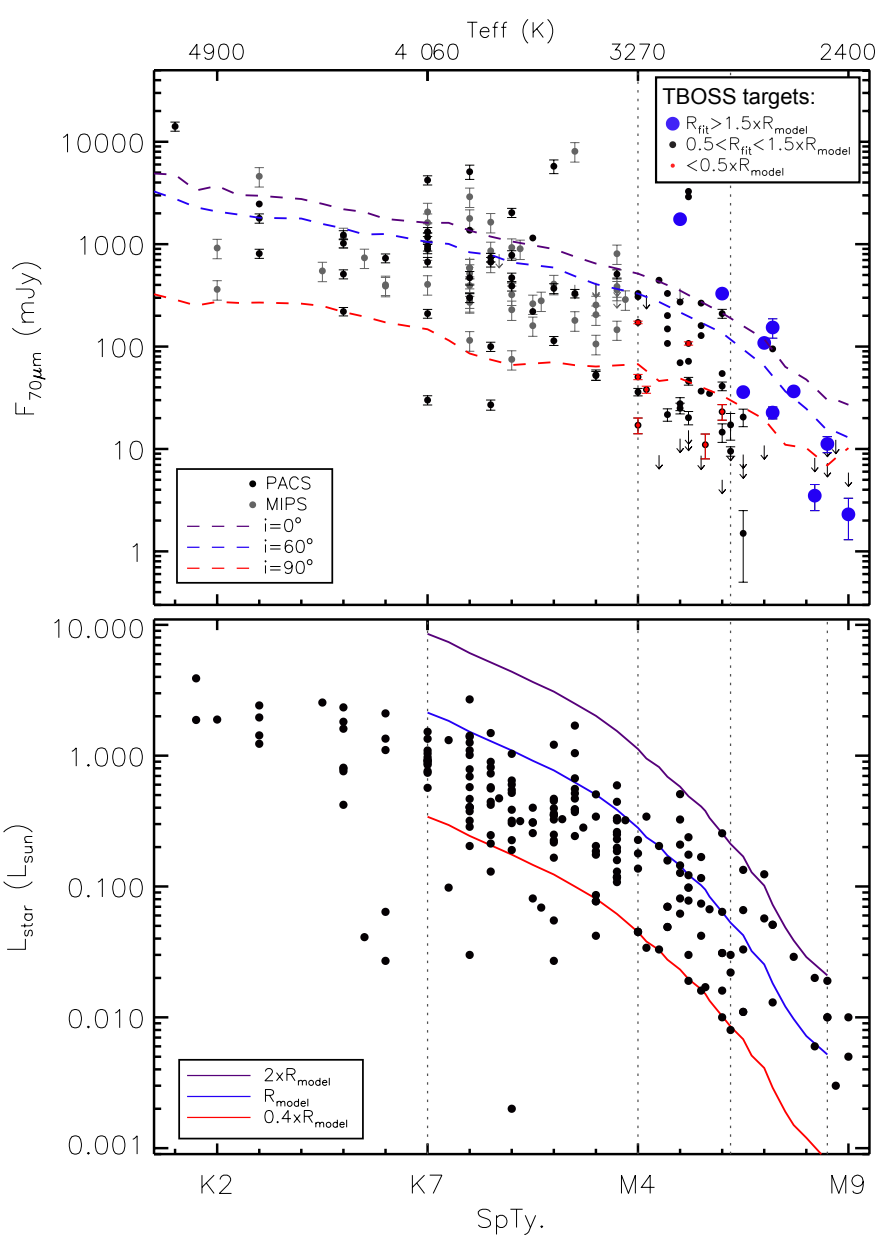

Fig. 7. Top: $70 \mu \mathrm{m}$ flux density shown as a function of spectral type for the Taurus Class II population, with spectral types spanning early $\mathrm{K}$ to late M. The vertical dotted lines mark the boundaries of the TBOSS sample (targets of spectral types M4+) and the stellar/substellar limit (M6.25). Flux densities measured from PACS and MIPS are represented by the black and gray points respectively. Additionally, for the detected TBOSS targets, the large blue points correspond to best-fit stellar radii of $>1.5$ times that of the evolutionary model values, and the small red points correspond to best-fit stellar radii of $<0.5$ times that of the evolutionary model values. Downwards arrows represent the $3 \sigma$ upper limits. The dashed lines represent the $70 \mu \mathrm{m}$ flux densities extracted from a test grid of model SEDs, generated with the radiative transfer code MCFOST and represent the flux densities for disk inclinations of face-on $\left(0^{\circ}\right.$; purple dashed-line), $60^{\circ}$ (blue dashed-line) and edge-on $\left(90^{\circ}\right.$; red dashed-line). Bottom: bolometric luminosities of the Taurus Class II population, with spectral types spanning early $\mathrm{K}$ to late $\mathrm{M}$. The luminosities of the TBOSS sample are derived from the best-fit stellar radii. The luminosities for targets of spectral type $<\mathrm{M} 4$ are from those reported in Andrews et al. (2013). The $1 \mathrm{Myr}$ isochrone from Baraffe et al. (1998) is represented with the blue solid line and the corresponding isochrones are shown for values of 2 and 0.4 times that of the model stellar radii (purple and red solid lines respectively).

The SEDs for all Class I targets are given in Appendix C. The PACS flux densities are at or near the peak power point for these embedded sources.

Among the Class II targets, $75 \%$ of the sample is detected in at least one of the Herschel channels, and the SEDs for the majority of these sources are given in Appendix D, Figs. D.1-D.4. Owing to to the difficulty in interpreting the SEDs for targets with unresolved companions, the SEDs for that category of target are plotted separately in Fig. D.1. The SEDs of the smaller

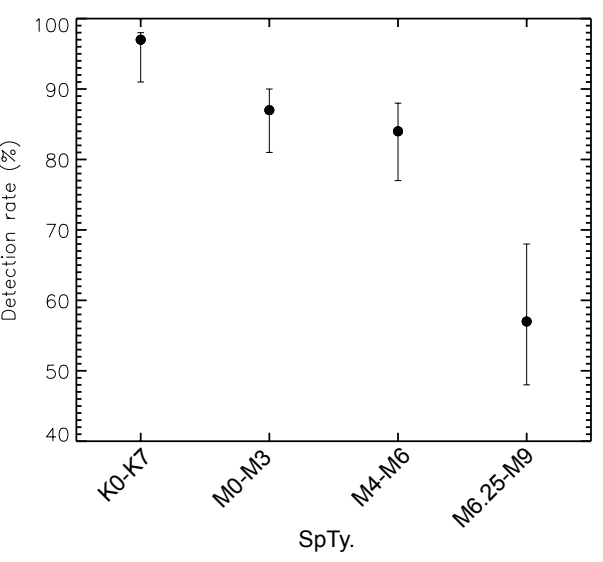

Fig. 8. Taurus Class II, $70 \mu \mathrm{m}$ detection rates for objects of spectral types K0-M9. The M4-M6 and M6.25-M9 detections rates are compiled from the TBOSS sample observed with Herschel PACS. The K0K7 and M0-M3 detections rates are compiled form the Herschel PACS observations reported in Howard et al. (2013) and the Spitzer MIPS observations reported in Rebull et al. (2010).

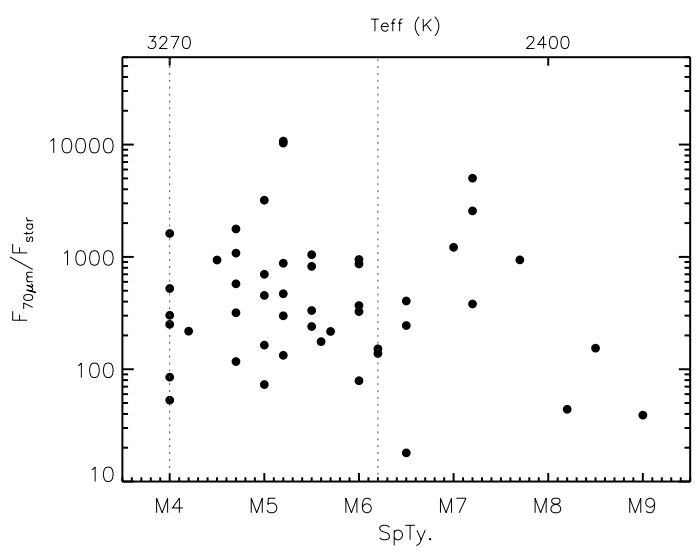

Fig. 9. $70 \mu \mathrm{m}$ disk excess fraction shown as a function of spectral type for the detected Class II objects within the TBOSS sample.

number of undetected Class II targets are shown in Fig. D.4. Based on the Herschel PACS data, a subset of the detected Class II targets are identified as candidate transition disk and candidate truncated disk objects; the SEDs for those targets are presented in Sects. 6.3 and 6.4, respectively.

In order to compare the shapes of the SEDs for different spectral types of the detected Class II targets, the median SED for each spectral type is shown in Fig. 11, and the median SEDs are scaled by the $J$-band flux density. As a result of the characteristic SED shape of Class II objects with edge-on disks (similar in appearance to Class I objects), targets with known edge-on disks - IRAS 04248+2612, J04381486+2611399, J04442713+2512164, and ZZ Tau-IRS (Padgett et al. 1999; Andrews et al. 2008; Luhman et al. 2007; Bouy et al. 2008; White \& Hillenbrand 2004) - are not included in the calculated median SEDs. Overall, the relative flux density at longer wavelengths declines as a function of spectral type, though there is no discontinuity at the stellar/substellar boundary.

Only four Class III targets have Herschel PACS detections, and the SEDs for these four sources are discussed further in Sect. 6.3. The remaining Class III targets with Herschel upper limits are shown in Appendix E. 

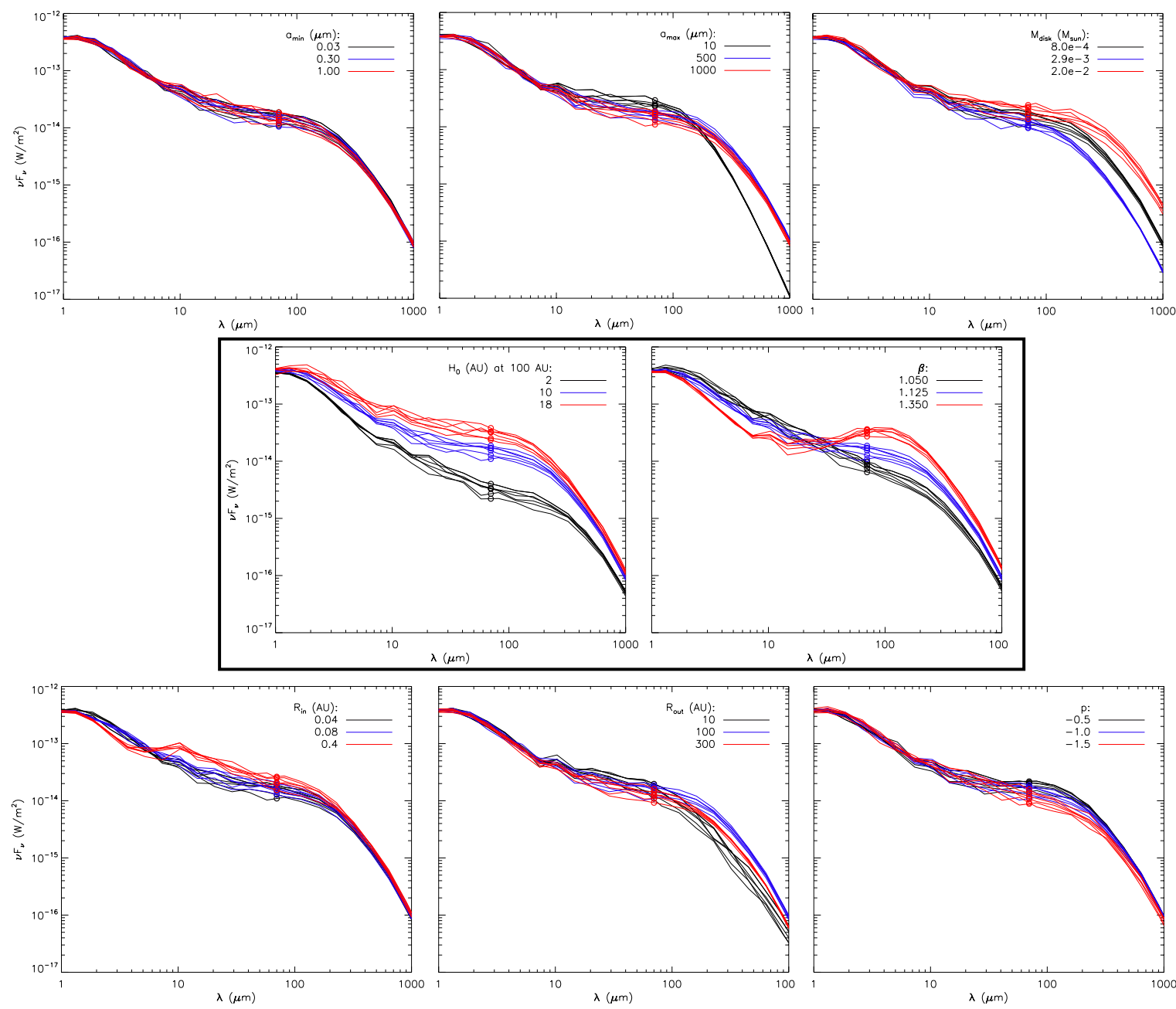

Fig. 10. SED visualization showing the impact that individual disk parameters have on excesses at $70 \mu \mathrm{m}$, the wavelength of which is highlighted on the SEDs with the open circle symbols. The SEDs were generated with the 3D radiative transfer code MCFOST (Pinte et al. 2006, 2009). In each individual panel, 3 models of SEDs were generated (black, blue and red lines), by varying one of the the eight disk parameters with values that are displayed in each panel. From left to right and top to bottom, the disk parameters are: minimum grain size $\left(a_{\min }\right)$, maximum grain size $\left(a_{\text {max }}\right)$, disk mass $\left(M_{\text {disk }}\right)$, scale height $\left(H_{0}\right)$ at a reference disk radius of $100 \mathrm{AU}$, disk flaring exponent $(\beta)$, inner radius $\left(R_{\text {in }}\right)$, outer radius $\left(R_{\text {out }}\right)$, and surface density exponent $(p)$. The SEDs were generated for a star of spectral type M4, and the stellar properties were set based on the temperature conversion of Luhman et al. (2003b) and the $1 \mathrm{Myr}$ evolutionary models of Baraffe et al. (1998), i.e., $T_{\text {eff }}=3300 \mathrm{~K}, R_{\text {star }}=1.65 R_{\odot}$, $M_{\text {star }}=0.29 M_{\odot}$. The model SEDs are shown at disk inclinations of $18^{\circ}, 32^{\circ}, 41^{\circ}, 49^{\circ}, 57^{\circ}$ and $63^{\circ}$ (where $0^{\circ}$ is for a face-on disk inclination), for each value of the varied disk parameters. Disk geometry; inclination, $H_{0}$ and $\beta$ are seen to have the largest impact on the emission at $70 \mu \mathrm{m}$.

\subsection{Candidate transition disks}

Transition disks represent a more advanced stage of disk evolution in which material in the disk has dissipated, either marked by an inner hole (e.g., Calvet et al. 2005), or a homologous depletion (Currie et al. 2009). A number of physical processes have been suggested to clear disk material, including photoevaporation (Alexander et al. 2006), disruption from companions (Artymowicz \& Lubow 1994), and grain growth (Dullemond \& Dominik 2004). The shape of the SED - specifically spectral indices between pairs of wavelengths - or spectral line features, or the absence of gas have been used to identify transition disks and later stage debris disks (Muzerolle et al. 2010; Kim et al. 2013). Within the TBOSS sample, 4 transition disks are known (Currie \& Sicilia-Aguilar 2011; Cieza et al. 2012), along with 2 debris/photoevaporative disks (Currie \& Sicilia-Aguilar 2011). Details of the previously known TBOSS transition disk and debris disk objects are listed in Table 8 .

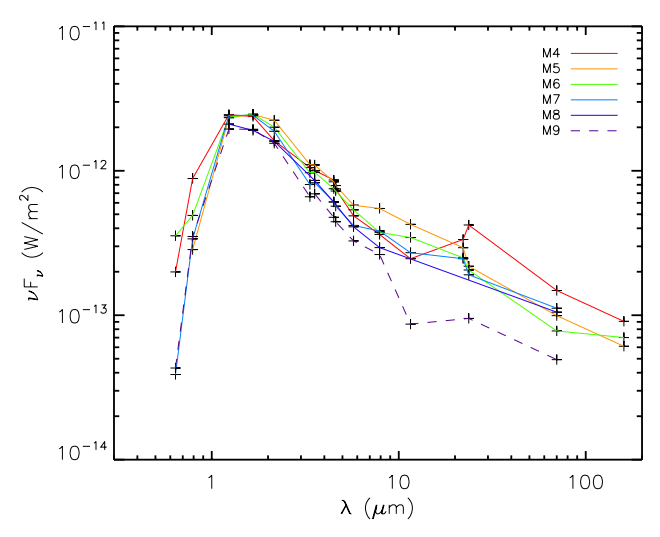

Fig. 11. Median SEDs of the Class II TBOSS targets detected with Herschel PACS. The median SEDs are normalized by the $J$-band flux density, and shown per spectral type for M4-M8. The normalized SED of the one, M9 detected target (KPNO 6; Harvey et al. 2012) is shown and represented by the purple, dashed line. 
Table 8. Transition disks within the TBOSS sample.

\begin{tabular}{lccccc}
\hline \hline 2MASS & Other name & Class & Sp Ty. & Transition disk type & Ref. \\
\hline J04144730+2646264 & FP Tau & 2 & M4 & Homologously depleted & $(1)$ \\
J04190110+2819420 & V410 X-ray 6 & 2 & M4.5 & Giant planet-forming & $(2)$ \\
J04161210+2756385 & $\ldots$ & 2 & M4.75 & Candidate & $(3)$ \\
J04322210+1827426 & MHO 6 & 2 & M4.75 & Candidate & $(3)$ \\
J04202555+2700355 & $\ldots$ & 2 & M5.25 & Candidate, (PD/IH) & $(3),(1)$ \\
J04210934+2750368 & $\ldots$ & 2 & M5.25 & Grain-growth dominated & $(2)$ \\
J04284263+2714039 & $\ldots$ & 2 & M5.25 & Grain-growth dominated & $(2)$ \\
J04403979+2519061 & $\ldots$ & 2 & M5.25 & Debris disk & $(2)$ \\
J04213459+2701388 & $\ldots$ & 2 & M5.5 & Candidate & $(3)$ \\
J04292971+2616532 & FW Tau A+B+C & 3 & M4 & Photoevaporating/Circumbinary & $(2)$ \\
J04295422+1754041 & & 3 & M4 & Candidate & $(3)$ \\
J04332621+2245293 & XEST 17-036 & 3 & M4 & Candidate & $(3)$ \\
J04354203+2252226 & XEST 08-033 & 3 & M4.75 & Candidate & $(3)$ \\
\hline
\end{tabular}

Notes. ${ }^{(a)}$ The evolutionary status for this target, previously reported in the literature was identified as inconclusive, being either a primordial disk (PD) object or a transition disk object with an inner hole (IH).

References. (1) Currie \& Sicilia-Aguilar (2011); (2) Cieza et al. (2012); (3) this work.

A series of spectral indices $\left(\alpha=-\frac{\operatorname{dog}\left(v F_{v}\right)}{\operatorname{dlog}(v)}\right)$ as a function of effective temperature calculated over wavelength ranges from the 3.6-8.0 $\mu \mathrm{m}$ IRAC bands to the $160-1300 \mu \mathrm{m}$ bands are plotted in 7 panels in Fig. 12 for the full Taurus Class II and Class III population with detections. Over the 3.6-8.0 $\mu \mathrm{m}$ wavelength range, the known transition disk SEDs have steeply declining slopes and occupy the region at the extreme of the Class II distribution and overlapping with Class III members. The transition disks again have a distinct distribution in the SED power law index over the $24-70 \mu \mathrm{m}$ range; the majority of the slopes are flat to rising. Based on the $24-70 \mu \mathrm{m}$ spectral index, we have identified a new population of 7 additional transition disk candidates with rising slopes, and these new systems are noted in Table 8 . Amongst these 7 transition disk candidates, J04202555+2700355 was previously investigated (Currie \& Sicilia-Aguilar 2011) and an inconclusive evolutionary status (primordial disk or transition disk with inner hole) was reported based on SED modeling results. XEST 17036 and XEST 08-033 are considered to be debris disks based on their $\alpha_{3.6-8.0 \mu \mathrm{m}}$ values that are consistent with those of previously reported debris/photoevaporative disks in the literature. Furthermore, XEST 17-036 - is remarkable in that it is among the few spatially resolved targets. Our selection criteria and classification for the targets that we identify to be candidate transition/debris disks is highlighted in the spectral index plot shown in Fig. 13. Based on conservative estimates of transition and debris disk objects reported in the literature, targets with $\alpha_{24-70 \mu \mathrm{m}}>0$, and $-2 \lesssim \alpha_{3.6-8.0 \mu \mathrm{m}} \lesssim-1$ are identified as candidate transition disk objects, and targets with $\alpha_{3.6-8.0 \mu \mathrm{m}} \lesssim-2.5$ are identified as debris disk objects. The SEDs of the known and candidate transition disks are shown in Fig. 14. The 5 new transition disk candidates have spectral types ranging from M4-M5.5.

From the total number of transition disk objects, it is possible to determine the typical statistical lifetime for this evolutionary stage. The estimated transition disk lifetime of $\sim 0.45 \mathrm{Myr}$ for targets of spectral types K5-M5 was calculated from the ratio of the number of transition disk objects to that of primordial disk objects, and assuming that the average primordial disk lifetime is $\sim 3$ Myr (Luhman et al. 2010). Following the same procedure we obtain a ratio of $9 / 52$ (accounting for the 4 Class III objects that are detected at $70 \mu \mathrm{m}$ and/or $160 \mu \mathrm{m}$ ), and an estimated lifetime of $\sim 0.5 \mathrm{Myr}$ for targets of spectral types M4-M9, similar to the K5-M5 value.

\subsection{Candidate truncated disks}

A total of 15 Class II targets with $24 \mu$ m flux densities were not detected with the Herschel $70 \mu \mathrm{m}$ PACS maps, including one target not observed with Herschel, that is undetected at $70 \mu \mathrm{m}$ with Spitzer MIPS. The non-detections are not simply the latest spectral type targets, but include examples extending to M4.25. To estimate the average flux density for this small subset of undetected Class II members, all the maps were combined to form a single $70 \mu \mathrm{m}$ map shown in Fig. 15, and the flux density of the faint combination source was measured as $3.0 \pm 0.5 \mathrm{mJy}$. This exercise was repeated for the $160 \mu$ m maps in which a $3 \sigma$ upper limit of $14 \mathrm{mJy}$ was measured in the combined map. Both the individual detection limit and these estimates of the average flux densities at $70 \mu \mathrm{m}$ and $160 \mu \mathrm{m}$ are plotted on the SEDs for the 17 Class II targets that are not detected at far-IR wavelengths with either Herschel PACS or Spitzer MIPS (presented in Appendix D.4). For 3 of the these targets - CIDA 14, MHO 5 and $\mathrm{J} 04322415+2251083$ - the individual limit alone indicates that the slope of the SED has a power law index $\left(\alpha_{24-70 \mu \mathrm{m}}\right)$ of -2.0 or steeper. Considering the lower value of the combined flux from all the non-detected targets, 5 Class II targets with $24 \mu \mathrm{m}$ detections and $70 \mu \mathrm{m}$ upper limits have slopes steeper than -2.0 .

One possible explanation for the shape of the Class II targets with exceptionally steep far-IR SED slopes is truncation of their disks. An example of a likely truncated disk in the $\epsilon$ Cha moving group around ET Cha was identified from a similarly steep SED index $\left(\alpha_{24-70 \mu \mathrm{m}}=-1.4\right)$ over wavelengths extending to the PACS bands combined with a non-detection of the $\mathrm{CO}(3-2)$ line (Woitke et al. 2011). In addition to the 3 Class II non-detected sources, there are 5 Class II targets with $70 \mu \mathrm{m}$ detections that also define a slope as steep as that of ET Cha - CFHT 12, FR Tau, KPNO 13, J04141188+2811535, and V410 Xray 1 resulting in a total of 8 candidate truncated disks. The SEDs of these candidate truncated disk targets are presented in Fig. 16. Follow-up high sensitivity $\mathrm{CO}$ spectral line observations of these steep slope SED targets could provide further evidence of truncated disks around these 8 Taurus targets. 

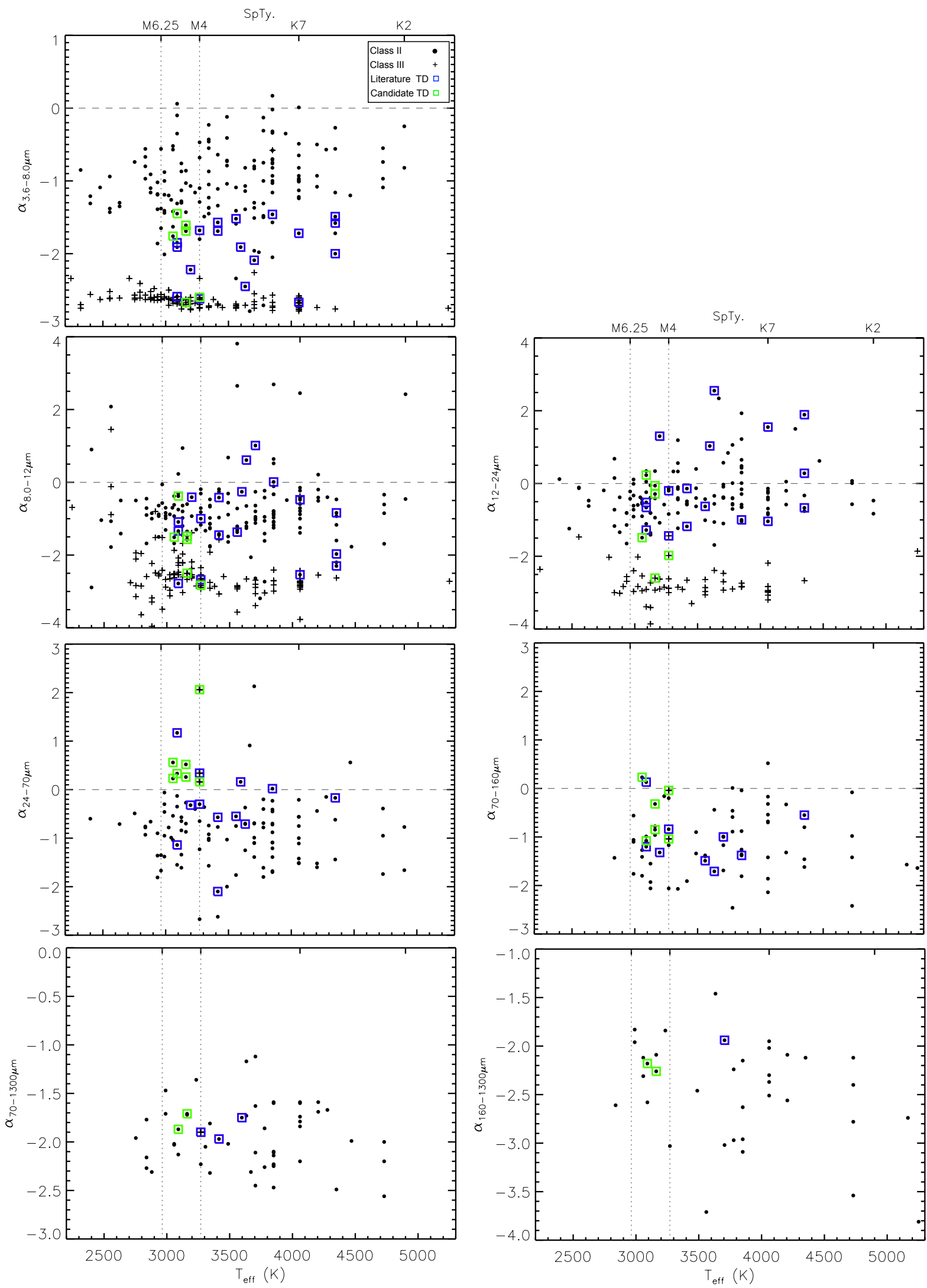

Fig. 12. Series of spectral index plots shown as a function of stellar effective temperature, merging the known Taurus Class II (filled circles) and Class III (crosses) members with; mid-IR emission (IRAC, MIPS and WISE), far-IR emission (PACS and MIPS), and $1.3 \mathrm{~mm}$ emission (for those targets not with no reported $1.3 \mathrm{~mm}$ observations, the nearest submm $/ \mathrm{mm}$ observation was scaled to $1.3 \mathrm{~mm}$ assuming $F_{v} \propto v^{2}$ ). Symbols leftwards of the vertical dotted lines, plotted at $T_{\text {eff }}=2963 \mathrm{~K}$ and $T_{\text {eff }}=3270 \mathrm{~K}$, indicate the substellar $(\leq \mathrm{M} 6.25)$ members and members of the TBOSS sample $(\leq \mathrm{M} 4)$, respectively. Blue squares enclose the targets identified in the literature with transition disks and green squares enclose the candidate transition disks objects identified within this paper. 


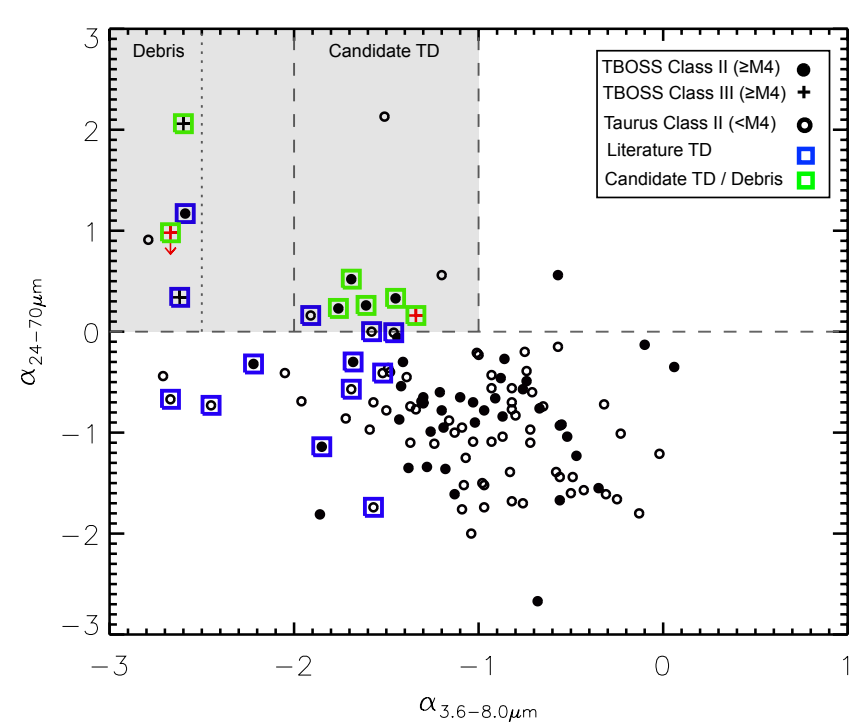

Fig. 13. Spectral index plot showing the Taurus Class II and Class III members with mid-IR and far-IR excess. The spectral indices; $\alpha_{3.6-8.0 \mu \mathrm{m}}$ and $\alpha_{24-70 \mu \mathrm{m}}$ are used to identify candidate targets with transition disks (TDs; points bounded by the horizontal and vertical dashed lines) and debris disks (points bounded by the horizontal dashed and vertical dotted lines). Targets identified in the literature with TDs, and the candidate TD/debris disks reported here, are enclosed by blue and green squares respectively. J04295422+1754041 (red cross) was unobserved with IRAC and MIPS, and the spectral indices are calculated from the WISE $3.4 \mu \mathrm{m}$ and $12 \mu \mathrm{m}$ flux densities, and the WISE $22 \mu \mathrm{m}$ and PACS $70 \mu \mathrm{m}$ flux densities. XEST 08-033 (red cross with downward arrow) was only detected with PACS at $160 \mu \mathrm{m}$, and the 24-70 spectral index shown is calculated from the $70 \mu \mathrm{m}$ upper limit.

\subsection{Impact of companions}

Many of the 154 M4+ TBOSS sample have been observed by infrared direct imaging from the ground (Duchêne et al. 2004; Luhman et al. 2009a), high resolution imaging employing spacebased measurements with HST (White \& Ghez 2001; Kraus et al. 2006; Todorov et al. 2014), ground-based speckle imaging (Kohler \& Leinert 1998; Konopacky et al. 2007), and aperture masking or AO imaging (Kraus \& Hillenbrand 2009b; Kraus et al. 2011). The results of the PACS observations presented here therefore provide the opportunity to assess the similarity or difference in the frequency or typical flux density level of disks for targets with and without companions. Whilst the comprehensive analysis of multiplicity and completeness for those TBOSS targets with existing imaging data will be addressed in a forthcoming coming paper, the subset of known and candidate transition disk and candidate truncated disk objects represent interesting cases in which to initially assess the multiplicity rate, since the inner holes of misidentified transition disks may be due to the presence of a companion that gravitationally truncates the inner radii of a circumbinary disk (e.g., Ireland \& Kraus 2008). For binary systems with semi-major axis $a$, the outer edges of the circumstellar disk(s) are expected to be truncated to disk radii of $\sim 0.2-0.5 a$ (Artymowicz \& Lubow 1994). Details of the targets for which imaging observations have been investigated for companions are given in Table 7 , along with the corresponding literature reference. The binary systems that were detected with the PACS blue channel $(70 \mu \mathrm{m}$ or $100 \mu \mathrm{m})$ among the categories of transition, truncated, mixed pairs, and extended objects are given in Table 7.
Amongst the transition disk objects identified within the TBOSS sample, 10 of 13 targets possess high resolution imaging data, from which a binary disk fraction of $40_{-13}^{+16} \%$, and single disk fraction of $60_{-16}^{+13} \%$ is calculated. Similarly, amongst the truncated disk objects identified within the TBOSS sample 5 of 8 targets possess high resolution imaging data, from which a binary disk fraction of $40_{-16}^{+22} \%$, and single disk fraction of $60_{-22}^{+16 \%}$ is calculated. There is no significant difference between the binary and single star disk fractions for the transition disk and truncated disk objects.

\subsection{Mixed pair systems}

Mixed pairs form an interesting subset of multiple systems comprised of one component with a disk and one component without a disk. Studies of spatially resolved binary systems in Taurus investigating inner disk signatures such as $\mathrm{H} \alpha$ emission and near-IR excesses reveal that mixed pair systems are rare, representing $\sim 15-20 \%$ of the population (e.g., Prato \& Simon 1997; Duchêne et al. 1999; Hartigan \& Kenyon 2003; Monin et al. 2007). Furthermore, considering these inner disk diagnostics, the component with the disk is equally likely to be the primary or secondary (Monin et al. 2007; Daemgen et al. 2012). With interferometric submm/ $\mathrm{mm}$ observations sensitive to the bulk of the disks in Class II binaries in Taurus and Ophiuchus (Jensen \& Akeson 2003; Patience et al. 2008; Harris et al. 2012), 19 pairs have been spatially resolved, consisting of 14 systems with only a circumprimary disk, 3 systems of both circumprimary and circumsecondary disks, and 2 systems with only a circumsecondary disk. In each of the systems with only a circumsecondary disk in the submm/mm, the primaries are actually close binary pairs with separations of 5-45 AU (Kraus et al. 2011). Among the TBOSS sample, two examples of Class II/Class III mixed systems are identified - J04414565+2301580/J04414489+2301513 and $\mathrm{J} 04554757+3028077 / \mathrm{J} 04554801+3028050$. The PACS maps and SEDs of these systems are shown in Fig. 17. In each case, the secondary is the disk-bearing component based on the far-IR emission from the Herschel data. Based on high resolution imaging, both the primary and secondary of the J04414565/J04414489 system posses close companions, while neither the primary nor secondary of the J04554757/J04554801 system has a close companion (Kraus et al. 2011; Todorov et al. 2014). The angular separations of the two mixed pair systems, and the additional separations for the close companions of the J04414565/J04414489 system are listed in Table 7 . In the $\mathrm{J} 04414565 / \mathrm{J} 04414489$ system, the host of the secondary disk is a very low mass M8.5 ( $\left.20 M_{\text {Jup }}\right)$ brown dwarf approaching the planetary mass regime.

\subsection{Candidate proto-brown dwarfs in Taurus}

Proto-brown dwarfs are cores of substellar mass, at the Class 0 or Class I stage of low mass star-formation (Adams et al. 1987; Andre et al. 1993; Pound \& Blitz 1993). Observable diagnostics, revealed in characterizing the earliest stages of brown dwarf evolution, can be used to distinguish between the number of different brown dwarf formation scenarios such as embryo ejection (Bate et al. 2002), disk fragmentation (Stamatellos \& Whitworth 2011) or photoionization from nearby massive stars (Whitworth \& Zinnecker 2004). It is also noteworthy that the first discovery of a pre-brown dwarf core - a self-gravitating starless core of dust and gas with a mass in the brown-dwarf regime - is located in the dense Ophiuchus L1688 cloud (André et al. 2012), 

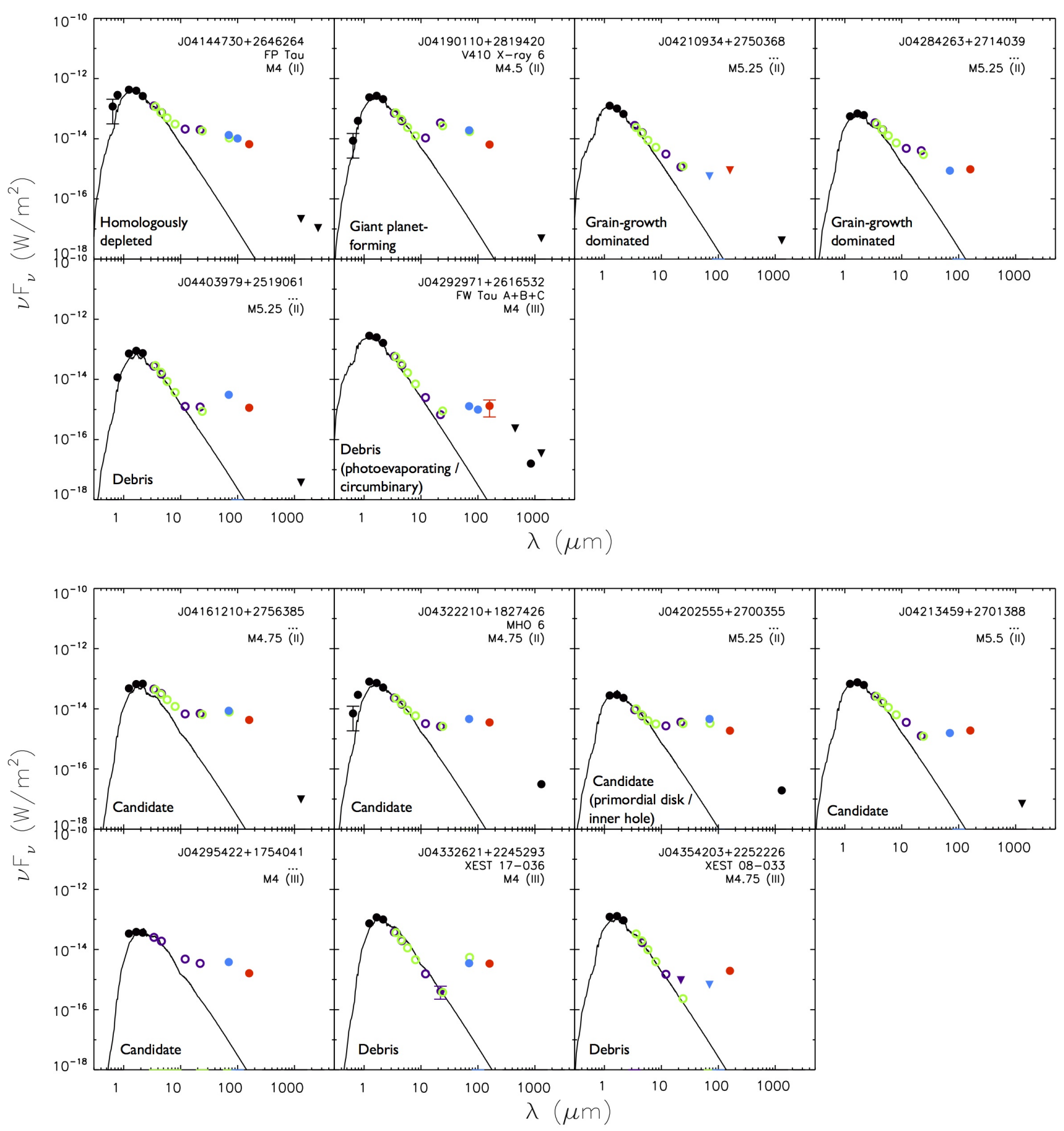

Fig. 14. SEDs showing the targets with transition disks (TD) previously reported in the literature (top panel) and the new candidate targets with TDs identified in this paper (bottom panel). Additionally, J04202555+2700355 for which the evolutionary status was previously reported as inconclusive (Currie \& Sicilia-Aguilar 2011), is identified as a candidate TD here. For those targets identified in the literature the TD types, compiled from Currie \& Sicilia-Aguilar (2011) and Cieza et al. (2012), are displayed on each SED. The candidate TDs are identified by the rising spectral index from $24-70 \mu \mathrm{m}\left(\alpha_{24-70 \mu \mathrm{m}}>0\right)$. In addition to the positive $\alpha_{24-70 \mu \mathrm{m}}$ indices for XEST 17-036 and XEST 08-033, the $\alpha_{3.6-8.0} \mu \mathrm{m}$ indices are typical for those of Class III (debris disk) objects. The target name, spectral type, and spectral class are labeled in each SED. The observed broadband photometry (see also Appendix A) is compiled from optical $\left(R_{\mathrm{C}}, I_{\mathrm{C}}\right)$, and near-IR (2MASS; $\left.J H K_{\mathrm{S}}\right)$ wavelengths (black points), the mid-IR (IRAC and WISE; green and purple open circles respectively), the far-IR (MIPS, PACS blue and red channels; green open circles, blue and red points respectively) and submm-mm wavelengths (black points). $3 \sigma$ upper limits are represented by the downwards triangles. The best-fit atmospheric model are displayed for each target.

indicating that these objects can form in environmental conditions denser than Taurus. Given the divergence of possible formation models, it is important to identify the youngest population of proto-brown dwarfs. Among the Taurus population, only two Class 0 objects are known, and none have spectral types in the M4-L0 TBOSS range, however, there are several candidate proto-brown dwarfs among the Class I TBOSS range, and each case is described below. 


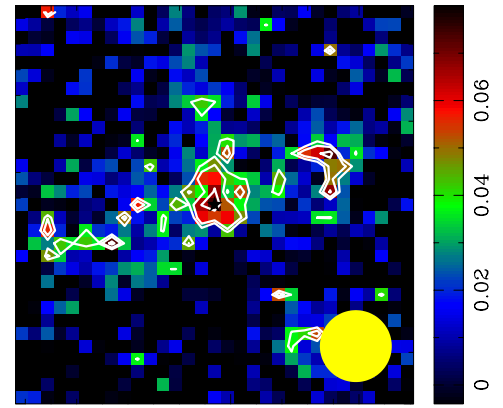

Fig. 15. PACS blue channel $(70 \mu \mathrm{m})$ combined map of the undetected Class II objects. The map is $30^{\prime \prime}$ in size along each axis. The beam size is represented by the yellow circle in the bottom, right-hand corner and the black star marks the peak position in the map. Contour levels are plotted at 4,5 and $6 \sigma$. The scale bar shows the intensity of the map in $\mathrm{mJy} /$ pixel.

Three sources within the TBOSS sample may be considered as proto-brown dwarf candidates - [GKH94] 41, IRAS 04191+1523 B, and L1521F-IRS. [GKH94] 41 has been identified to be a disk-dominated source based on IRS spectra (Furlan et al. 2011), and is therefore at a more evolved stage than the Class I classification that has been adopted here (Luhman et al. 2010). The accretion rate for L1521F-IRS is estimated to be low and likely towards the end of its main (Class 0) accretion phase (Bourke et al. 2006). IRAS 04191+1523 B is the secondary component of a 6.' 1 binary (Duchêne et al. 2004), making the data at wavelengths longer than $8 \mu \mathrm{m}$ contaminated by the primary emission. Given the quiescent phase of accretion and the bolometric temperature consistent with M6-M8 (Luhman et al. 2010), L1521F-IRS and IRAS 04191+1523 B will remain substellar unless a large amount of material is added to the central source. From the SED of L1521F-IRS, this candidate protobrown dwarf appears heavily embedded and the slope over the $160 \mu \mathrm{m}$ to $1.3 \mathrm{~mm}$ range is $\alpha=-1.8$, which is markedly shallower than the other isolated Class I TBOSS targets ( $\alpha$ typically ranging from -3.4 to -2.7 ). The SED slope of L1521F-IRS is similar to ISM grains and may indicate that this target has only recently transitioned from a Class 0 to Class I object.

\subsection{Comparison with other star-forming regions}

Comparison of the results of large-scale disk population studies performed in different star-forming regions and in young clusters can provide an indication of the impact of environmental factors and evolutionary trends. From IR observations over the $J$ - to $L$-band of young populations spanning the $0.3-5$ Myr age range, a typical disk lifetime of $\$ 3 \mathrm{Myr}$ was inferred from the decline in IR excess frequency (Haisch et al. 2001). From longer wavelength Spitzer observations of Taurus extending to $24 \mu \mathrm{m}$, the disk fraction (defined by the ratio of Class II to Class II+III members) as a function of central object mass was similar to Chameleon I, but higher than IC 348, and the lower stellar density of Taurus and Chameleon was suggested as a possible explanation (Luhman et al. 2010). Submillimeter population studies comparing Taurus and Ophiuchus measured similar disk fractions and average disk masses for both regions (Andrews \& Williams 2005, 2007). These existing IR and submm population studies mainly focus on the earlier spectral type members outside the TBOSS sample range.

Given the unique sensitivity and scope of the TBOSS survey, it is difficult to compare the Taurus M4+ far-IR disk results with those of other star-forming regions. The most analogous study is a recent Herschel investigation of 43 brown dwarfs (taken as objects with M6+ spectral types) in the L1688 cloud of Ophiuchus, for which a disk fraction of $\sim 0.3$ at $70 \mu \mathrm{m}$ is revealed (Alves de Oliveira et al. 2013). Considering the M6+ targets in the TBOSS sample, the detected disk fraction is also $\sim 0.3$ (22 of 67 targets), however the sensitivity of the TBOSS observations is much deeper. Restricting the TBOSS M6+ disks to those that could have been detected in the Ophiuchus study ( $~ 225 \mathrm{mJy}$ ) yields a lower disk fraction of $\sim 0.15$, suggesting that the average disk flux density for a Taurus member at or below the stellar/substellar boundary is lower than for an equivalent object in Ophiuchus.

\section{Summary}

Of the 154 total M4-L0 members of the Taurus star-forming region, we report new $70 \mu \mathrm{m}$ and $160 \mu \mathrm{m}$ flux densities or upper limits for 134 targets and combine the results with other Herschel and Spitzer programs to compile the PACS measurements for 153 targets comprising the TBOSS sample. For the 148 TBOSS targets not contaminated by emission from unresolved earlier spectral type primaries, the detection rates at $70 \mu \mathrm{m}$ with PACS and MIPS were calculated for the different evolutionary classes and spectral type ranges. Considering all M4-L0 TBOSS targets, $100 \%$ of Class I, 75\% of Class II, and $4 \%$ of Class III TBOSS targets were detected. Dividing the Class II targets into M4-M6 members above the substellar limit and M6.25+ brown dwarfs, the far-IR detection rate is significantly higher for the lowest mass stars relative to brown dwarfs. Although the disk frequencies are different, the distributions of disk-to-central-object far-IR flux density are indistinguishable above and below the substellar limit for disk targets, based on a K-S test.

To obtain a benchmark first census of far-IR disk emission across the full stellar and substellar population of any starforming region, the TBOSS results were combined with Spitzer and Herschel surveys of the earlier spectral type members. The $70 \mu \mathrm{m}$ flux density as a function of spectral type declines with a trend similar to the decline in flux density of the central object, and the range of flux density at a given spectral type span at least an order of magnitude. Using the radiative transfer code MCFOST to model the range of Herschel flux densities, the dominant parameters influencing the PACS bands was found to be the disk inclination, scale height, and flaring index. The majority of the detected Taurus Class II population can be constrained with flared disks $(\beta=1.125)$, with scale heights of $10 \mathrm{AU}$ at a reference disk radius of $100 \mathrm{AU}$ (equivalent to an opening angle of $5^{\circ}$ ), and with disk inclinations from face- to edge-on.

For all TBOSS targets, the SEDs were constructed by joining the far-IR data with results from optical to mid-IR surveys and submm/mm flux densities when available. Based on the spectral indices over the $24 \mu \mathrm{m}$ to $70 \mu \mathrm{m}$ range, 5 new candidate transition disks were identified. While the new and known transition disks have a distinct distribution in the slope over the $24 \mu \mathrm{m}$ to $70 \mu \mathrm{m}$ range, the spectral indices for these objects at longer wavelengths are indistinguishable from the values for the general Class II population. Another set of 8 targets have very steep SED slopes over the $24 \mu \mathrm{m}$ to $70 \mu \mathrm{m}$ range, possibly indicating truncated disks such as has been suggested for the disk around ET Cha (Woitke et al. 2011). Two other unusual targets are diskbearing secondary components of mixed systems. Finally, the overall Taurus substellar disk frequency is similar to that of the 


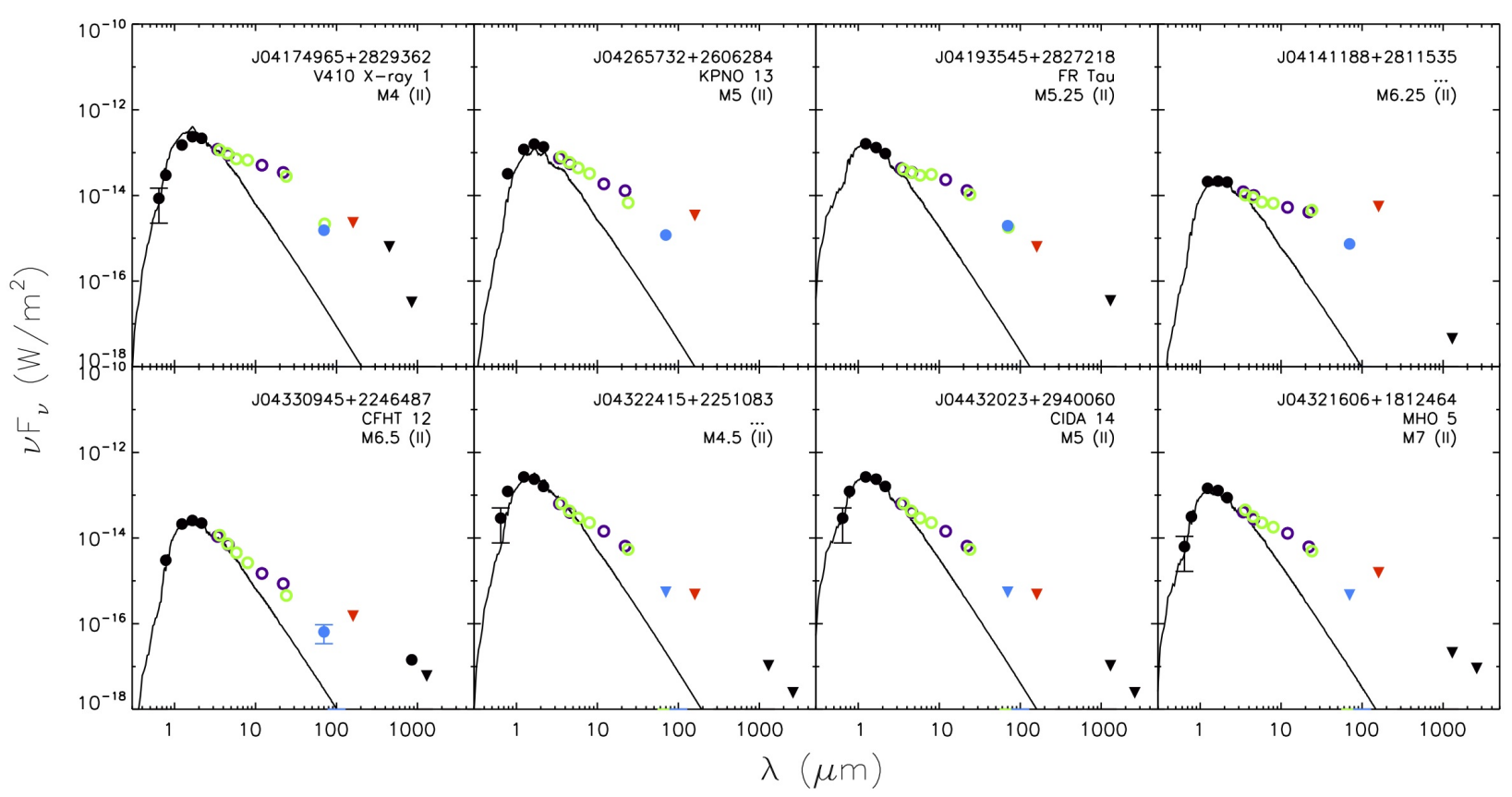

Fig. 16. SEDs of the eight candidate targets with truncated disks, identified from the steeply declining spectral index from $24-70 \mu \mathrm{m}$

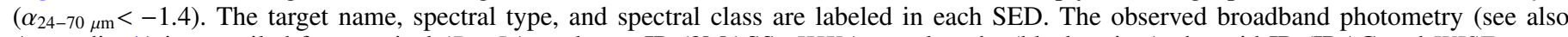
Appendix A) is compiled from optical $\left(R_{\mathrm{C}}, I_{\mathrm{C}}\right)$, and near-IR (2MASS; $\left.J H K_{\mathrm{S}}\right)$ wavelengths (black points), the mid-IR (IRAC and WISE; green and purple open circles respectively), the far-IR (MIPS, PACS blue and red channels; green open circles, blue and red points respectively) and submm-mm wavelengths (black points). $3 \sigma$ upper limits are represented by the downwards triangles. The best-fit atmospheric model are displayed for each target.
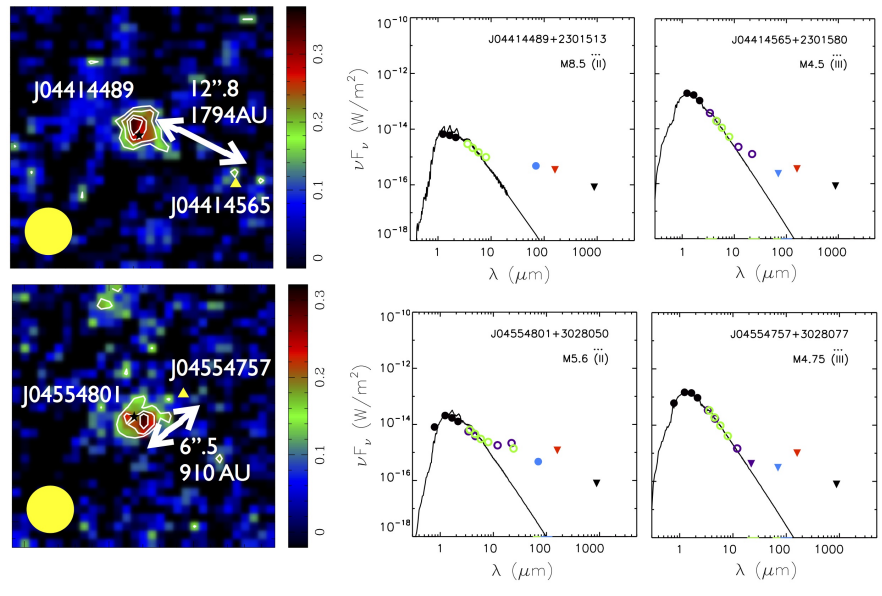

Fig. 17. PACS blue channel $(70 \mu \mathrm{m})$ maps (left) for the two mixed pair systems identified within the TBOSS sample; J0441489+2301513/ $\mathrm{J} 04414565+2301580$ and $\mathrm{J} 04554801+3028050 / \mathrm{J} 04554757+3028077$, and the corresponding SEDs (right). The black stars in the maps show the 2MASS positions of the detected Class II secondaries (J0441489 and J04554801), and the yellow triangles mark the 2MASS positions of the undetected Class III primaries (J04414565 and J04554757). Contours are plotted at $3,4,5$ and $6 \sigma$ and the PACS $70 \mu$ m beam size is represented by the yellow circle in the bottom, left corner of each map. Target name, spectral type, and spectral class are displayed in the SEDs. Broadband photometry, compiled from optical-mm wavelengths and the underlying best-fit atmospheric models are displayed in the SEDs.

brown dwarfs in Ophiuchus, but the average disk flux density for a Taurus M6+ target is lower than for a comparable object in Ophiuchus.

Acknowledgements. J. Bulger and J. Patience gratefully acknowledge support to Exeter from the STFC (ST/F0071241/1, ST/H002707/1), support from the Faculty of the European Space Astronomy Centre (ESAC), and support for this work was provided through an award issued by JPL/Caltech. C. Pinte acknowledges funding from the European Commission's 7th Framework Program (contract PERG06-GA-2009-256513) and from Agence Nationale pour la Recherche (ANR) of France under contract ANR-2010-JCJC-0504-01. We thank Dr. G. Matthews, Dr. F. Ménard, Dr. C. Pinte and collaborators from the GASPS team for providing some of their results prior to publication. We thank the staff at the NASA Herschel Science Center for their informative responses to queries regarding PACS data. This research has made use of the SIMBAD database, operated at CDS, Strasbourg, France. This publication makes use of data products from the Two Micron All Sky Survey, which is a joint project of the University of Massachusetts and the Infrared Processing and Analysis Center/California Institute of Technology, funded by the National Aeronautics and Space Administration and the National Science Foundation. This publication makes use of data products from the Wide-field Infrared Survey Explorer, which is a joint project of the University of California, Los Angeles, and the Jet Propulsion Laboratory/California Institute of Technology, funded by the National Aeronautics and Space Administration. HIPE is a joint development by the Herschel Science Ground Segment Consortium, consisting of ESA, the NASA Herschel Science Center, and the HIFI, PACS and SPIRE consortia. This publication makes use of observations made with the NASA/ESA Hubble Space Telescope, obtained from the data archive at the Space Telescope Science Institute. STScI is operated by the Association of Universities for Research in Astronomy, Inc. under NASA contract NAS 5-26555.

\section{References}

Adams, F. C., Lada, C. J., \& Shu, F. H. 1987, ApJ, 312, 788

Alexander, R. D., Clarke, C. J., \& Pringle, J. E. 2006, MNRAS 369, 229

Allard, F., Hauschildt, P. H., Alexander, D. R., Tamanai, A., \& Schweitzer, A. 2001, ApJ, 556, 357

Allard, F., Guillot, T., Ludwig, H.-G., et al. 2003, Brown Dwarfs, 211, 325

Allard, F., Homeier, D., \& Freytag, B. 2011, 16th Cambridge Workshop on Cool Stars, 448, 91

Alves de Oliveira, C., Ábrahám, P., Marton, G., et al. 2013, A\&A, 559, 126

Andre, P., Ward-Thompson, D., \& Barsony, M. 1993, ApJ, 406, 122

André, P., Ward-Thompson, D., \& Greaves, J. 2012, Science, 337, 69

Andrews, S. M., \& Williams, J. P. 2005, ApJ, 631, 1134

Andrews, S. M., \& Williams, J. P. 2007, ApJ, 671, 1800

Andrews, S. M., Liu, M. C., Williams, J. P., \& Allers, K. N. 2008, ApJ, 685, 1039

Andrews, S. M., Rosenfeld, K. A., Kraus, A. L., \& Wilner, D. J. 2013, ApJ, 771, 129 
Artymowicz, P., \& Lubow, S. H. 1994, ApJ, 421, 651

Baraffe, I., Chabrier, G., Allard, F., \& Hauschildt, P. H. 1998, A\&A, 337, 403 Basu, S., \& Vorobyov, E. I. 2012, ApJ, 750, 30

Bate, M. R., Bonnell, I. A., \& Bromm, V. 2002, MNRAS, 332, L65

Bate, M. R., Bonnell, I. A., \& Bromm, V. 2003, MNRAS, 339, 577

Beckwith, S. V. W., Sargent, A. I., Chini, R. S., \& Guesten, R. 1990, AJ, 99, 924

Bertout, C., Robichon, N., \& Arenou, F. 1999, A\&A, 352, 574

Bessell, M. S. 1979, ASP, 91, 589

Boden, A. F., Torres, G., Sargent, A. I., et al. 2007, ApJ, 670, 1214

Boden, A. F., Torres, G., Duchêne, G., et al. 2012, ApJ, 747, 17

Boss, A. P. 1997, Science, 276, 1836

Bourke, T. L., Myers, P. C., Evans, N. J., et al. 2006, ApJ, 649, L37

Bouy, H., Huélamo, N., Pinte, C., et al. 2008, A\&A, 486, 877

Briceño, C., Hartmann, L., Stauffer, J., \& Martín, E. 1998, AJ, 115, 2074

Briceño, C., Calvet, N., Kenyon, S., \& Hartmann, L. 1999, AJ, 118, 1354

Briceño, C., Luhman, K. L., Hartmann, L., Stauffer, J. R., \& Kirkpatrick, J. D. 2002, ApJ, 580, 317

Calvet, N., D'Alessio, P., Watson, D. M., et al. 2005, ApJ, 630, L185

Chabrier, G., Baraffe, I., Allard, F., \& Hauschildt, P. 2000, ApJ, 542, 464

Chauvin, G., Lagrange, A.-M., Dumas, C., et al. 2004, A\&A, 425, L29

Cieza, L. A., Schreiber, M. R., Romero, G. A., et al. 2012, ApJ, 750, 157

Cohen, M., Wheaton, W. A., \& Megeath, S. T. 2003, AJ, 126, 1090

Currie, T., \& Sicilia-Aguilar, A. 2011, ApJ, 732, 24

Currie, T., Lada, C. J., Plavchan, P., et al. 2009, ApJ, 698, 1

Cutri, R. M., Skrutskie, M. F., van Dyk, S., et al. 2003, The IRSA 2MASS All-

Sky Point Source Catalog

Cutri, R. M., et al. 2012, VizieR On-line Data Catalog: II/311

Daemgen, S., Correia, S., \& Petr-Gotzens, M. G. 2012, A\&A, 540, 46

Dent, W. R. F., Thi, W. F., Kamp, I., et al. 2013, PASP, 125, 477

Dobashi, K., Uehara, H., Kandori, R., et al. 2005, PASJ, 57, 1

Duchêne, G., Monin, J.-L., Bouvier, J., \& Ménard, F. 1999, A\&A, 351, 954

Duchêne, G., Bouvier, J., Bontemps, S., André, P., \& Motte, F. 2004, A\&A, 427, 651

Dullemond, C. P., \& Dominik, C. 2004, A\&A, 421, 1075

Engelbracht, C. W., Blaylock, M., Su, K. Y. L., et al. 2007, PASP, 119, 994

Fazio, G. G., Hora, J. L., Allen, L. E., et al. 2004, ApJS, 154, 10

Francesco, J. D., Johnstone, D., Kirk, H., MacKenzie, T., \& Ledwosinska, E. 2008, ApJS, 175, 277

Furlan, E., Luhman, K. L., Espaillat, C., et al. 2011, ApJS, 195, 3

Gordon, K. D., Engelbracht, C. W., Fadda, D., et al. 2007, PASP

Guieu, S., Dougados, C., Monin, J.-L., Magnier, E., \& Martín, E. L. 2006, A\&A, 446, 485

Guieu, S., Pinte, C., Monin, J.-L., et al. 2007, A\&A, 465, 855

Haisch, K. E., Lada, E. A., \& Lada, C. J. 2001, ApJ, 553, L153

Haisch, K. E., Barsony, M., \& Tinney, C. 2010, ApJ, 719, L90

Harris, R. J., Andrews, S. M., Wilner, D. J., \& Kraus, A. L. 2012, ApJ, 751, 115

Hartigan, P., \& Kenyon, S. J. 2003, ApJ, 583, 334

Hartmann, L. 1997, Herbig-Haro Flows and the Birth of Stars; IAU Symp., 182, 391

Hartmann, L., Calvet, N., Allen, L., Chen, H., \& Jayawardhana, R. 1999, AJ, 118,1784

Harvey, P. M., Henning, T., Liu, Y., et al. 2012, ApJ, 755, 67

Hauschildt, P. H., Allard, F., \& Baron, E. 1999, ApJ, 512, 377

Hennebelle, P., \& Chabrier, G. 2008, ApJ, 684, 395

Henry, T. J. 1998, Brown dwarfs and extrasolar planets, 134, 28

Howard, A. W., Marcy, G. W., Bryson, S. T., et al. 2012, ApJS, 201, 15

Howard, C. D., Sandell, G., Vacca, W. D., et al. 2013, ApJ, 776, 21

Ireland, M. J., \& Kraus, A. L. 2008, ApJ, 678, L59

Jensen, E. L. N., \& Akeson, R. L. 2003, ApJ, 584, 875

Johnson, J. A., Aller, K. M., Howard, A. W., \& Crepp, J. R. 2010, PASP, 122, 905

Kenyon, S. J., \& Hartmann, L. 1995, ApJS, 101, 117

Kenyon, S. J., Dobrzycka, D., \& Hartmann, L. 1994, AJ, 108, 1872

Kim, K. H., Watson, D. M., Manoj, P., et al. 2013, ApJ, 769, 149

King, R. R., Goodwin, S. P., Parker, R. J., \& Patience, J. 2012a, MNRAS, 427, 2636

King, R. R., Parker, R. J., Patience, J., \& Goodwin, S. P. 2012b, MNRAS 421, 2025

Klein, R., Apai, D., Pascucci, I., Henning, T., \& Waters, L. B. F. M. 2003, ApJ, 593, L57

Kohler, R., \& Leinert, C. 1998, A\&A, 331, 977

Konopacky, Q. M., Ghez, A. M., Rice, E. L., \& Duchêne, G. 2007, ApJ, 663, 394
Kraus, A. L., \& Hillenbrand, L. A. 2009a, ApJ, 704, 531

Kraus, A. L., \& Hillenbrand, L. A. 2009b, ApJ, 703, 1511

Kraus, A. L., White, R. J., \& Hillenbrand, L. A. 2006, ApJ, 649, 306

Kraus, A. L., Ireland, M. J., Martinache, F., \& Hillenbrand, L. A. 2011, ApJ, 731, 8

Kraus, A. L., Ireland, M. J., Cieza, L. A., et al. 2014, ApJ, 781, 20

Luhman, K. L. 2000, ApJ, 544, 1044

Luhman, K. L. 2004, ApJ, 617, 1216

Luhman, K. L. 2006, ApJ, 645, 676

Luhman, K. L., \& Rieke, G. H. 1998, ApJ, 497, 354

Luhman, K. L., Briceño, C., Stauffer, J. R., et al. 2003a, ApJ, 590, 348

Luhman, K. L., Stauffer, J. R., Muench, A. A., et al. 2003b, ApJ, 593, 1093

Luhman, K. L., Lada, C. J., Hartmann, L., et al. 2005, ApJ, 631, L69

Luhman, K. L., Whitney, B. A., Meade, M. R., et al. 2006, ApJ, 647, 1180

Luhman, K. L., Adame, L., D’Alessio, P., et al. 2007, ApJ, 666, 1219

Luhman, K. L., Mamajek, E. E., Allen, P. R., \& Cruz, K. L. 2009a, ApJ, 703, 399

Luhman, K. L., Mamajek, E. E., Allen, P. R., Muench, A. A., \& Finkbeiner, D. P. 2009b, ApJ, 691, 1265

Luhman, K. L., Allen, P. R., Espaillat, C., Hartmann, L., \& Calvet, N. 2010, ApJS, 186, 111

Martín, E. L., Dougados, C., Magnier, E., et al. 2001, ApJ, 561, L195

Mathis, J. S. 1990, IN: Ann. Rev. Astron. Astrophys., 28, 37

Mayne, N. J., Harries, T. J., Rowe, J., \& Acreman, D. M. 2012, MNRAS, 423, 1775

Mohanty, S., Greaves, J., Mortlock, D., et al. 2013, ApJ, 773, 168

Monin, J.-L., Clarke, C. J., Prato, L., \& McCabe, C. 2007, Protostars and Planets V (Tucson university of Arizona Press), 395

Motte, F., \& André, P. 2001, A\&A, 365, 440

Muzerolle, J., Allen, L. E., Megeath, S. T., Hernández, J., \& Gutermuth, R. A. 2010, ApJ, 708, 1107

Osterloh, M., \& Beckwith, S. V. W. 1995, ApJ, 439, 288

Ott, S. 2010, Astronomical Data Analysis Software and Systems XIX, Proc. of a Conf. held October 4-8, 434, 139

Padgett, D. L., Brandner, W., Stapelfeldt, K. R., et al. 1999, AJ, 117, 1490

Padoan, P., Kritsuk, A., Michael, Norman, L., \& Nordlund, Å. 2005, Mem. Soc. Astron. It., 76, 187

Palla, F., \& Stahler, S. W. 1999, ApJ, 525, 772

Patience, J., Akeson, R. L., \& Jensen, E. L. N. 2008, ApJ, 677, 616

Phan-Bao, N., Lee, C.-F., Ho, P. T. P., \& Tang, Y.-W. 2011, ApJ, 735, 14

Pinte, C., Ménard, F., Duchêne, G., \& Bastien, P. 2006, A\&A, 459, 797

Pinte, C., Harries, T. J., Min, M., et al. 2009, A\&A, 498, 967

Poglitsch, A., Waelkens, C., Geis, N., et al. 2010, A\&A, 518, L2

Pollack, J. B., Hubickyj, O., Bodenheimer, P., et al. 1996, Icarus, 124, 62

Pound, M. W., \& Blitz, L. 1993, ApJ, 418, 328

Prato, L., \& Simon, M. 1997, ApJ, 474, 455

Reach, W. T., Megeath, S. T., Cohen, M., et al. 2005, PASP, 117, 978

Rebull, L. M., Padgett, D. L., McCabe, C.-E., et al. 2010, ApJS, 186, 259

Reid, I. N., \& Gizis, J. E. 1997, AJ v.113, 113, 2246

Reipurth, B., \& Clarke, C. 2001, AJ, 122, 432

Ricci, L., Isella, A., Carpenter, J. M., \& Testi, L. 2013, ApJ, 764, L27

Rieke, G. H., Young, E. T., Engelbracht, C. W., et al. 2004, ApJS, 154, 25

Schaefer, G. H., Dutrey, A., Guilloteau, S., Simon, M., \& White, R. J. 2009, ApJ, 701,698

Scholz, A., \& Jayawardhana, R. 2008, ApJ, 672, L49

Scholz, A., Jayawardhana, R., \& Wood, K. 2006, ApJ, 645, 1498

Skrutskie, M. F., Cutri, R. M., Stiening, R., et al. 2006, AJ, 131, 1163

Slesnick, C. L., Carpenter, J. M., Hillenbrand, L. A., \& Mamajek, E. E. 2006, AJ, 132, 2665

Song, I., Schneider, G., Zuckerman, B., et al. 2006, ApJ, 652, 724

Stamatellos, D., \& Whitworth, A. 2011, Computational Star Formation, 270, 223

Strom, K. M., \& Strom, S. E. 1994, ApJ, 424, 237

Thies, I., \& Kroupa, P. 2007, ApJ, 671, 767

Todorov, K. O., Luhman, K. L., Konopacky, Q. M., et al. 2014, ApJ, 788, 40

Torres, R. M., Loinard, L., Mioduszewski, A. J., \& Rodríguez, L. F. 2009, ApJ, 698,242

White, R. J., \& Basri, G. 2003, ApJ, 582, 1109

White, R. J., \& Ghez, A. M. 2001, ApJ, 556, 265

White, R. J., \& Hillenbrand, L. A. 2004, ApJ, 616, 998

Whitworth, A. P., \& Zinnecker, H. 2004, A\&A, 427, 299

Woitke, P., Riaz, B., Duchêne, G., et al. 2011, A\&A, 534, 44

Wright, E. L., Eisenhardt, P. R. M., Mainzer, A. K., et al. 2010, AJ, 140, 1868

Young, C. H., Shirley, Y. L., Evans, N. J., \& Rawlings, J. M. C. 2003, ApJS, 145, 111

Pages 20 to 34 are available in the electronic edition of the journal at http: //www . aanda. org 


\section{Appendix A: Compilation of literature flux densities}

The compilation of flux densities from optical-millimeter wavelength of the known 154 M4-L0 Taurus members is given in Tables A.1-A.4. Table A.1 lists the $R_{\mathrm{C}}, I_{\mathrm{C}}$, and $2 \mathrm{MASS} J H K_{\mathrm{S}}$ flux densities. Table A.2 lists the Spitzer IRAC, MIPS-1, and WISE All-Sky Data release flux densities. Table A.3 lists the Herschel PACS, and Spitzer MIPS-2 flux densities. Table A.4 lists the submillimeter and millimeter $(350 \mu \mathrm{m}-2.6 \mathrm{~mm})$ flux densities. The tables are only available at the CDS.

\section{Appendix B: Herschel PACS maps}

The Herschel PACS maps of the detected targets observed under this program shown in Figs. B.1-B.7, and are displayed in order of spectral class and type. Target name and spectral type are labeled in the top left-hand corner of each map. Both the blue channel $(70 \mu \mathrm{m})$ and red channel $(160 \mu \mathrm{m})$ maps are $60^{\prime \prime}$ in size along each axis. In each map the black star indicates the 2MASS position of the target. The 2MASS positions of known multiple companions are indicted by yellow triangles in the blue channel maps, and green triangles in the red channel maps. The scale bar shows the intensity of each map in units of mJy/pixel. Contour levels begin at $3 \sigma$ in all maps and are plotted at intervals depending on the source brightness. The PACS beam size is represented by the blue circle in the lower, right-hand corner of each map.
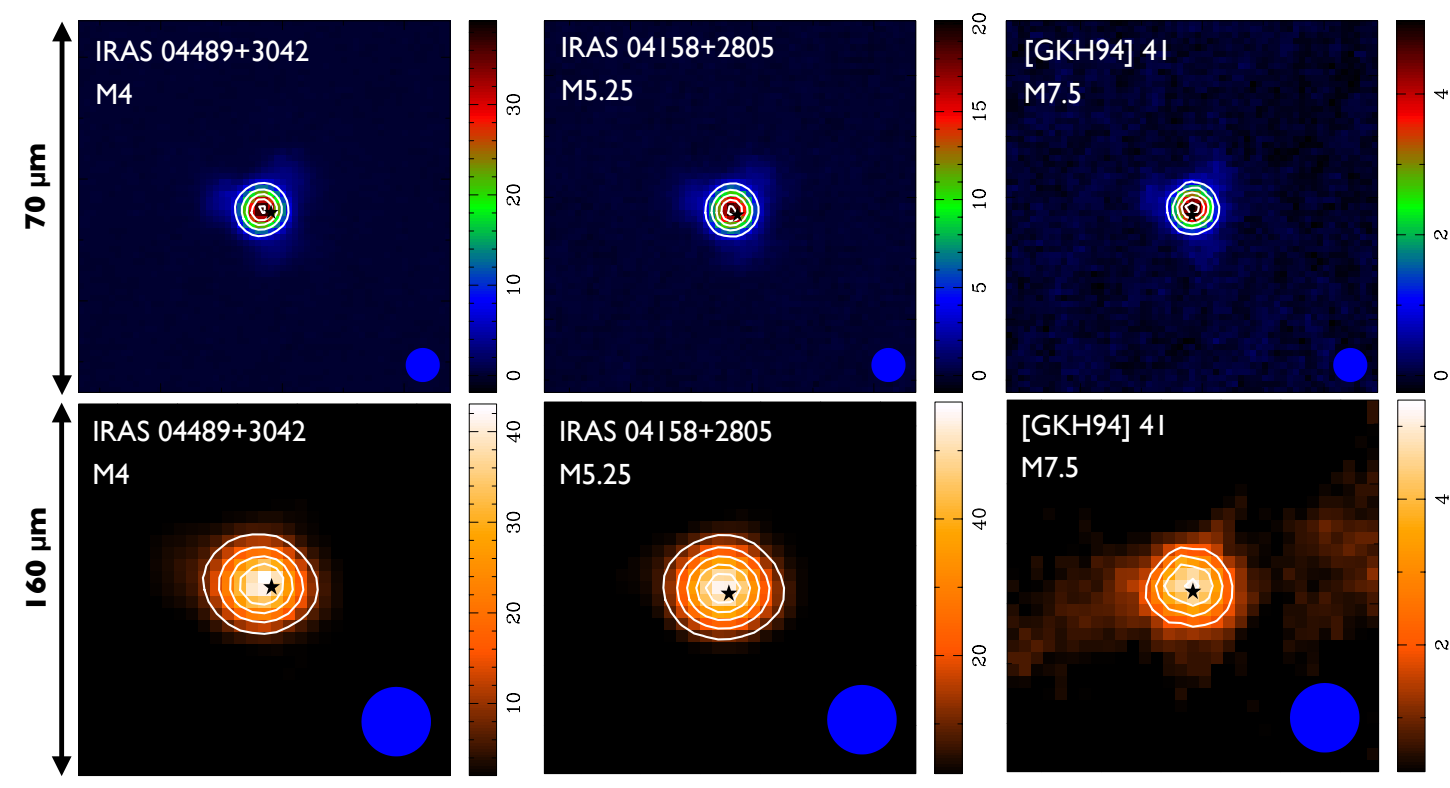

Fig. B.1. Class I objects with PACS blue and red channel detections. 
J. Bulger et al.: Disk emission across the stellar/substellar boundary in Taurus

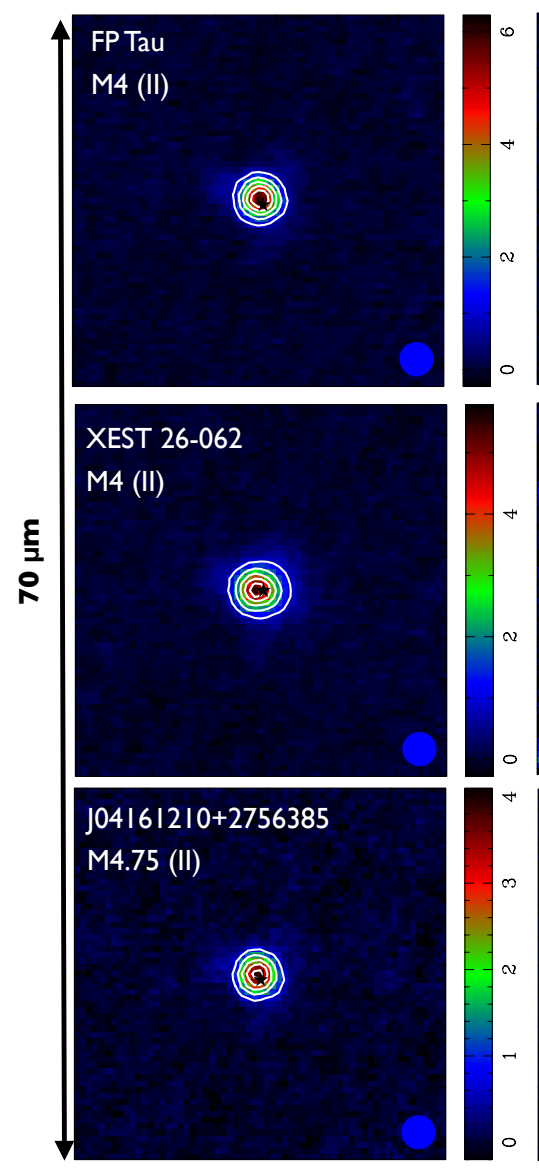

IRAS $04173+2812$ M4 (II)

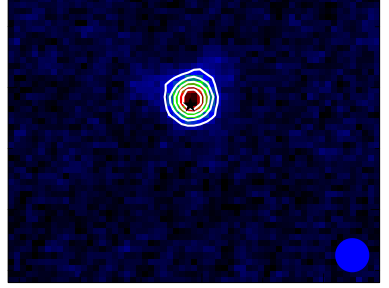

\section{CIDA 12}

M4 (II)

\section{(0)}
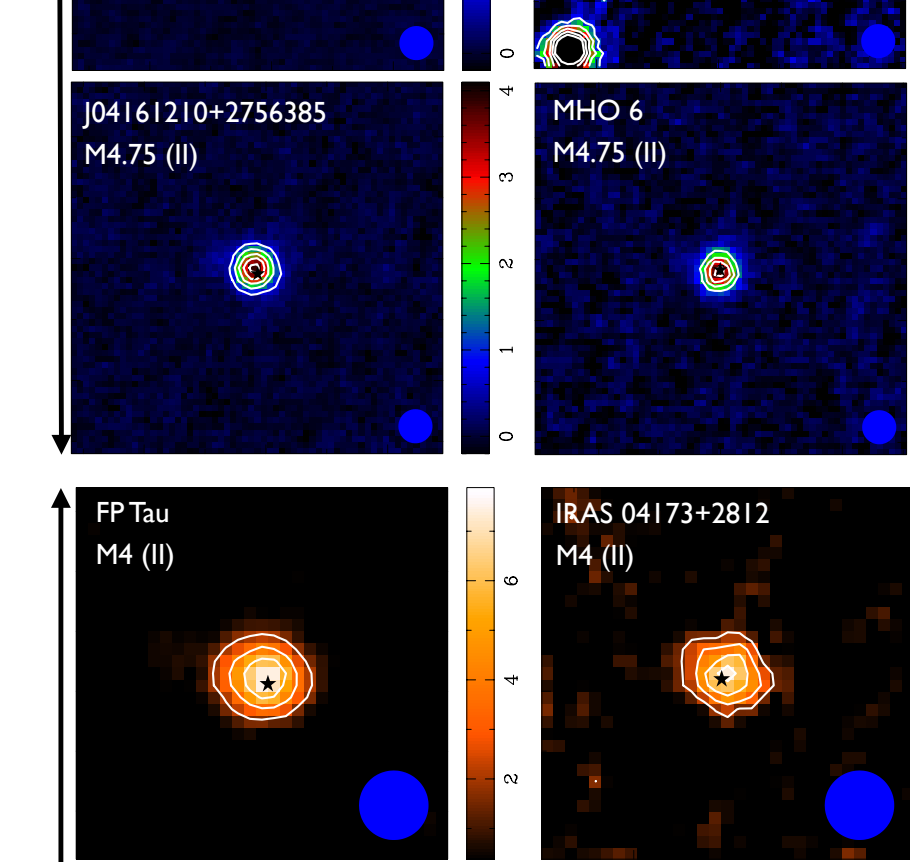

\section{IRAS $04173+2812$}

M4 (II)
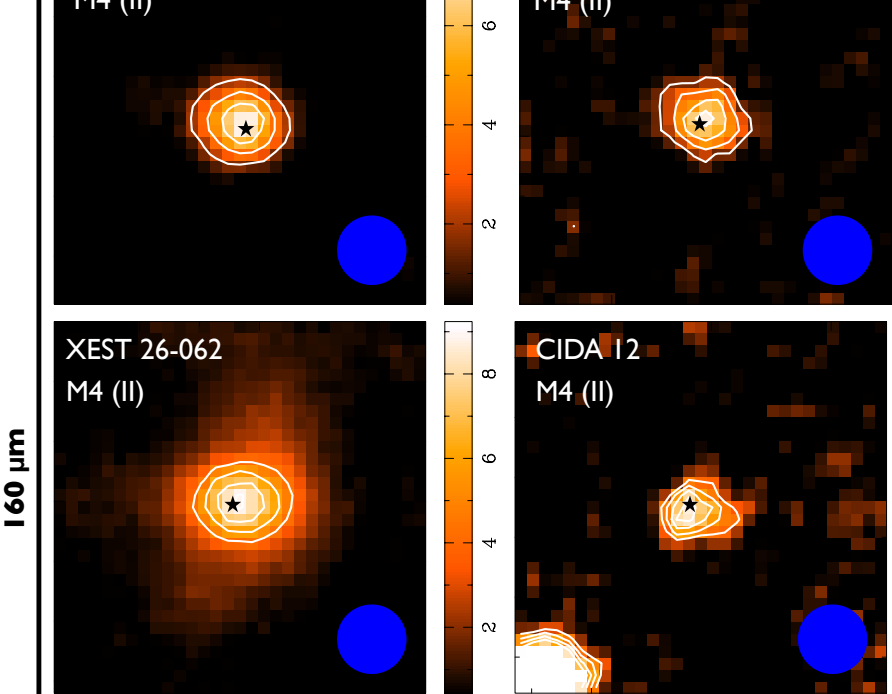

\section{J04161210+2756385} M4.75 (II)

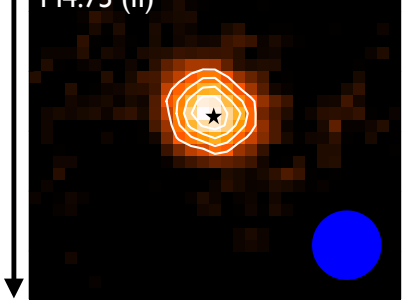

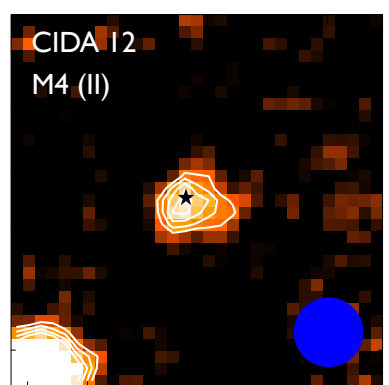

MHO 6

M4.75 (II)

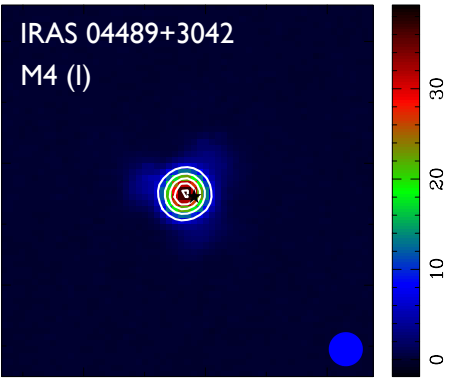

J04385859+233635I

M4.25 (II)

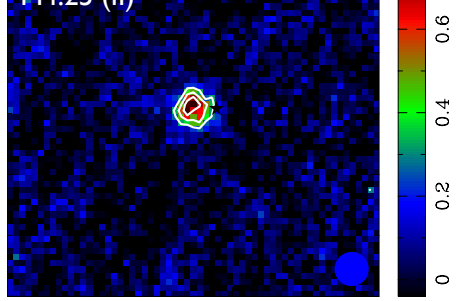

J04334465+26| 5005 M4.75 (II)

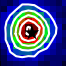

\section{IRAS 04489+3042}

M4 (I)
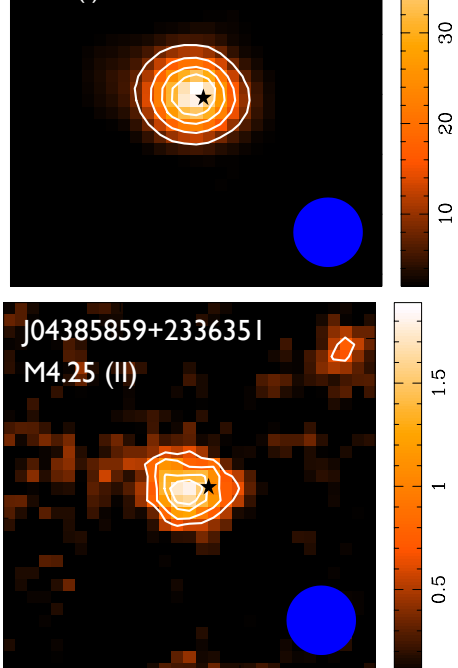

J04334465+26I5005

M4.75 (II)

Fig. B.2. Class II objects with PACS blue and red channel detections. 


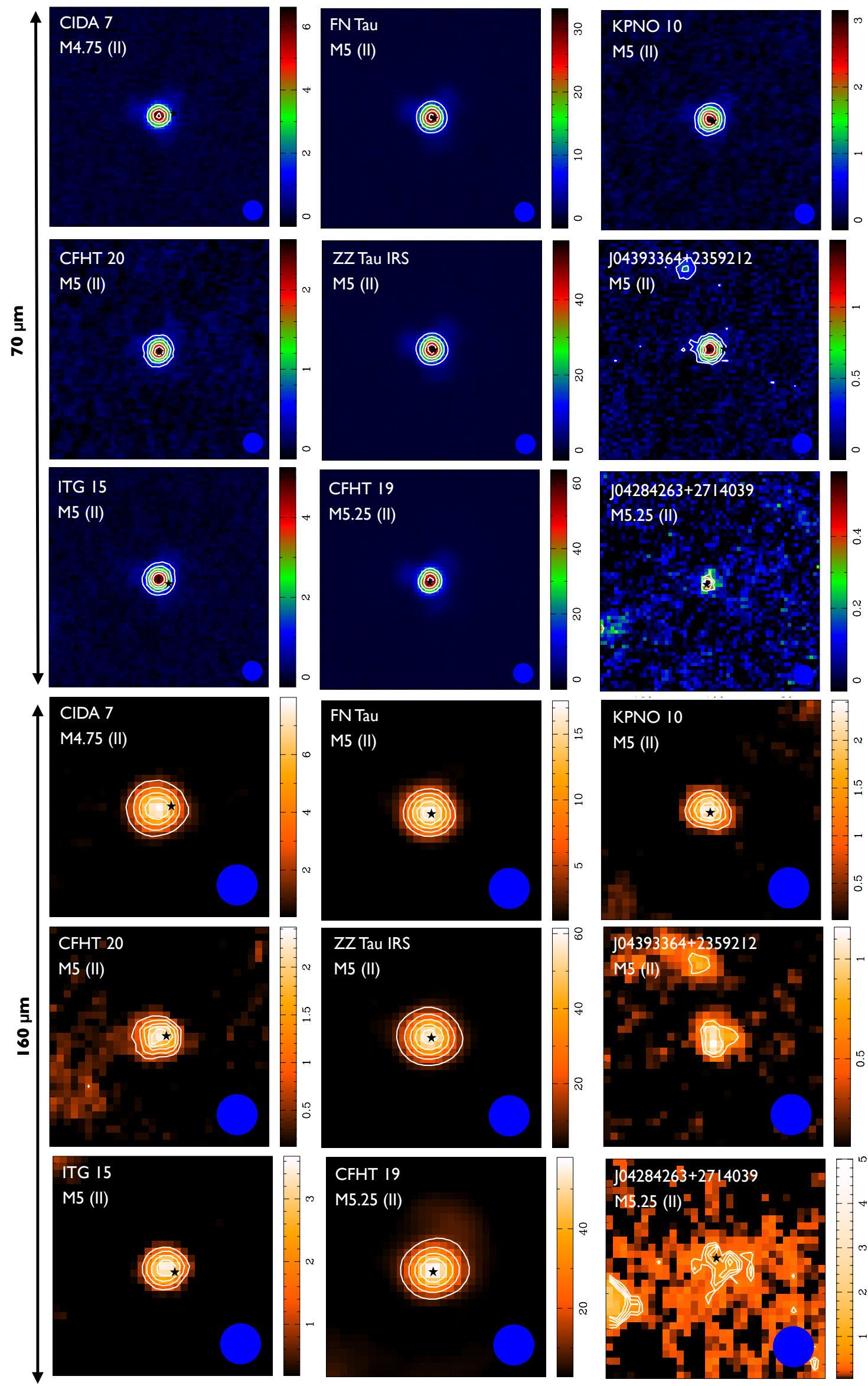

Fig. B.3. Class II objects with PACS blue and red channel detections. 
J. Bulger et al.: Disk emission across the stellar/substellar boundary in Taurus
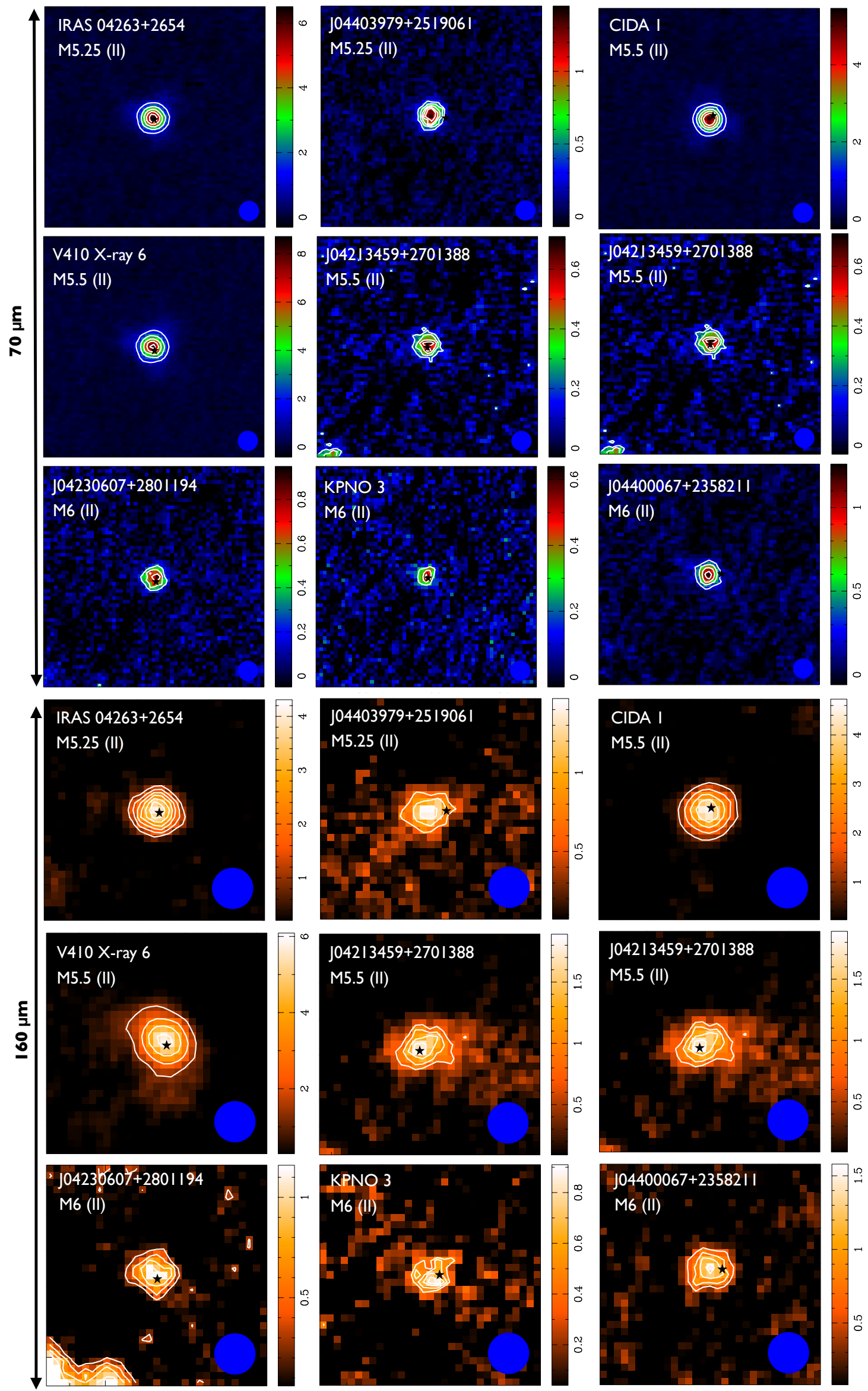

Fig. B.4. Class II objects with PACS blue and red channel detections. 
A\&A 570, A29 (2014)

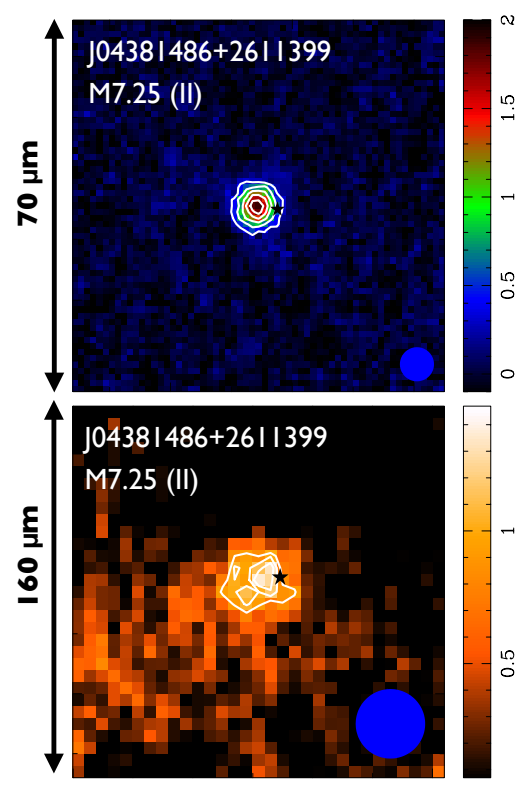

Fig. B.5. Class II objects with PACS blue and red channel detections. 
J. Bulger et al.: Disk emission across the stellar/substellar boundary in Taurus
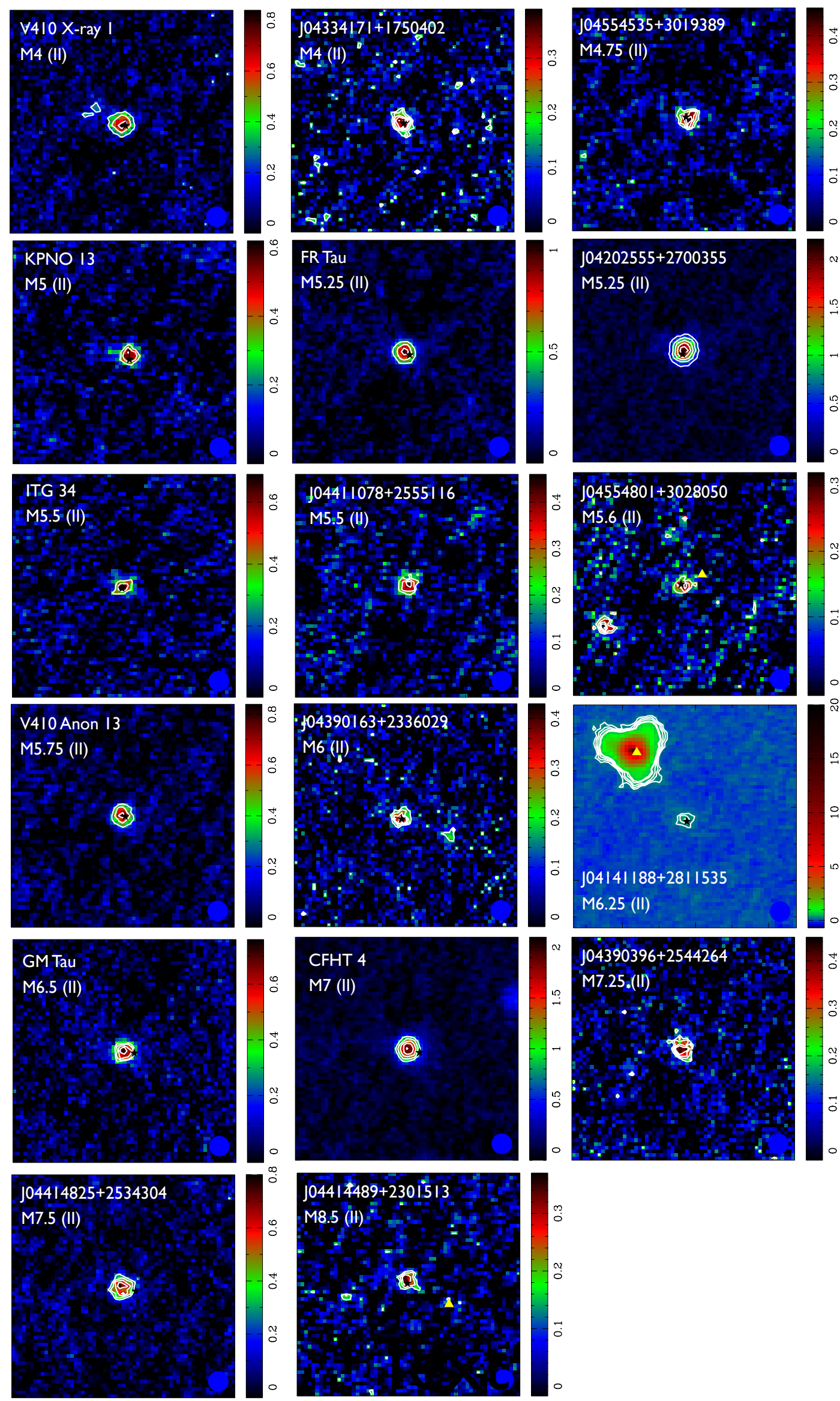

Fig. B.6. Class II objects with PACS blue channel detections only. 
A\&A 570, A29 (2014)

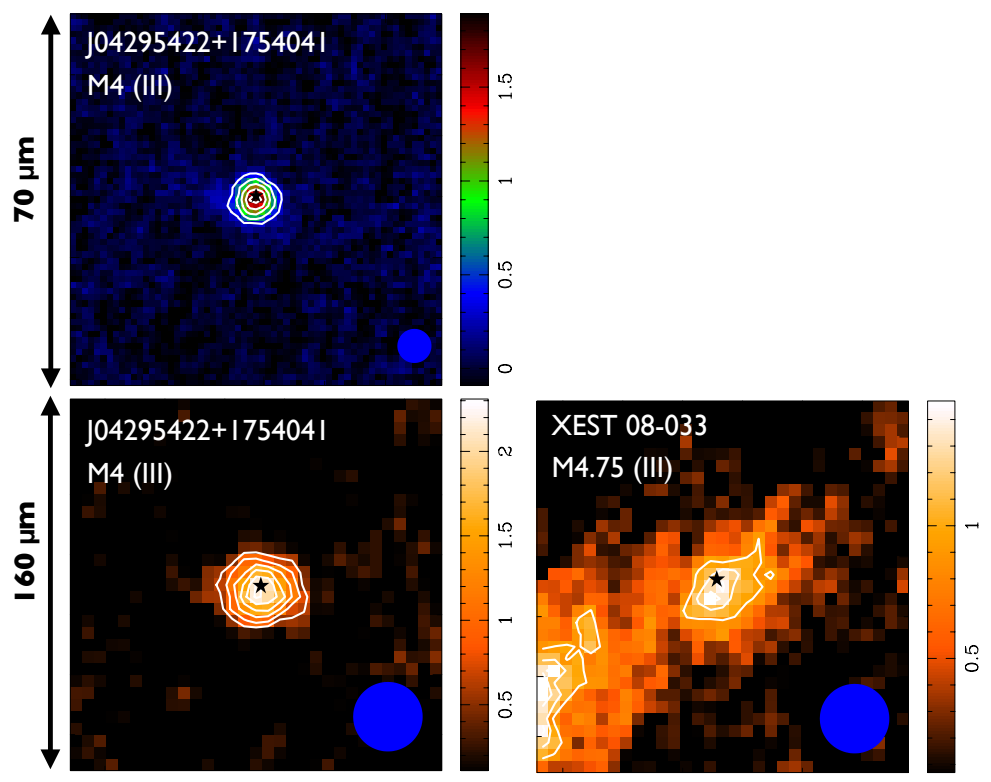

Fig. B.7. Class III objects with PACS blue and/or red channel detections. 


\section{Appendix C: SEDs of the TBOSS sample - Class I objects}

The SEDs for Class I objects of the TBOSS sample are shown in Fig. C.1. As IRAS 04191+1523 B is unresolved from IRAS 04191+1523 A with Herschel PACS, the SED(s) for this category of system(s) is shown in Fig. D.1.

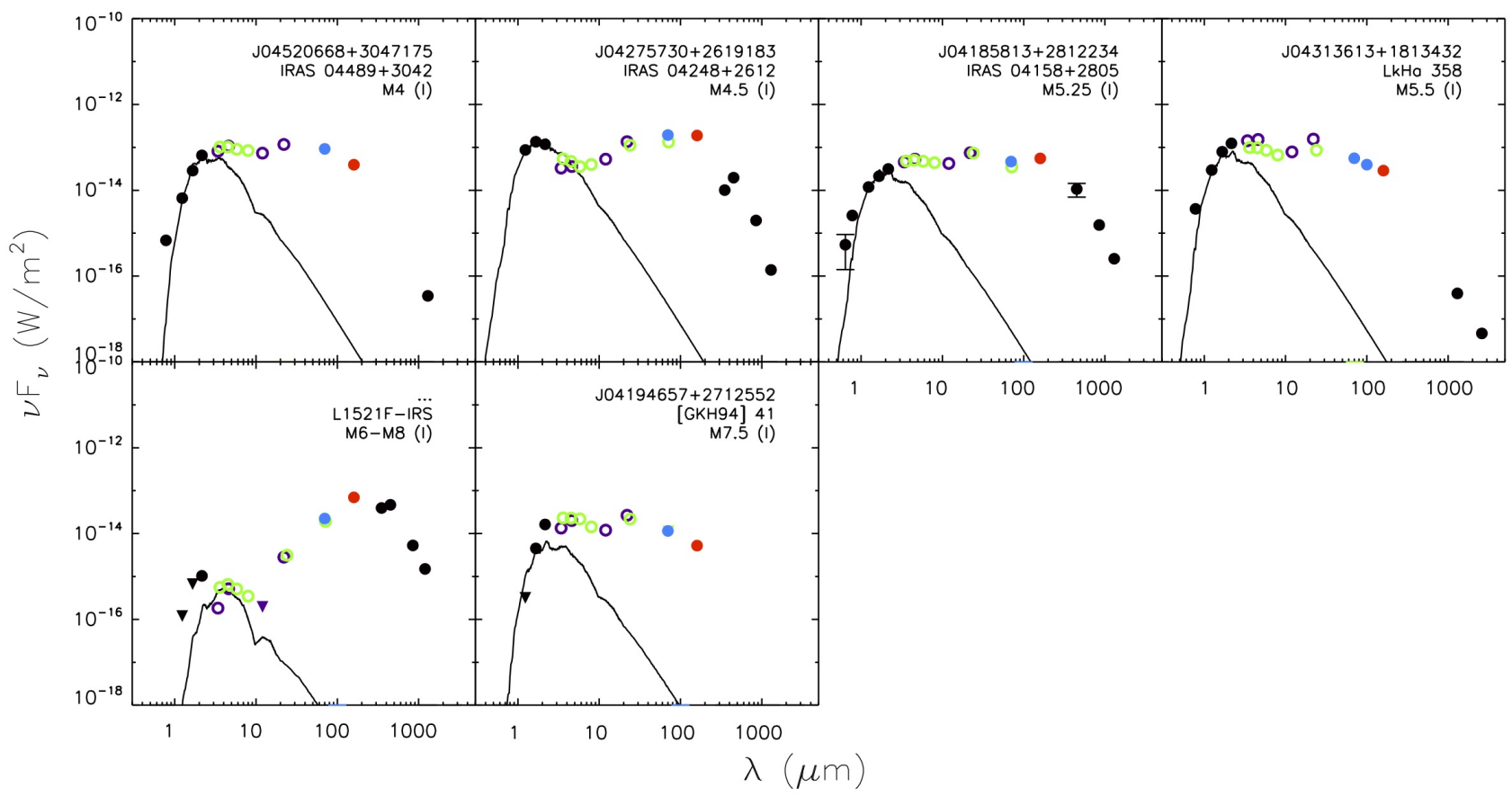

Fig. C.1. SEDs of the TBOSS of the Class I objects. The target name, spectral type, and spectral class are labeled in each SED. The observed broadband photometry is compiled from optical $\left(R_{\mathrm{C}}, I_{\mathrm{C}}\right)$, and near-IR (2MASS; JHK $\left.K_{\mathrm{S}}\right)$ wavelengths (black points), the mid-IR (IRAC and WISE; green and purple open circles respectively), the far-IR (MIPS, PACS blue and red channels; green open circles, blue and red points respectively) and submm-mm wavelengths (black points). $3 \sigma$ upper limits are represented by the downwards triangles. The best-fit atmospheric model are displayed for each target.

\section{Appendix D: SEDs of the TBOSS sample - Class II objects}

The additional SEDs for the Class II objects of the TBOSS sample are shown in Figs. D.1-D.4. Figure D.1 shows the targets that are in multiple systems of which are unresolved with Herschel PACS. Figures D.2 and D.3 shows the SEDs the Class II objects that are detected with Herschel PACS. Finally, Fig. D.4 shows the SEDs of the Class II objects that were undetected with Herschel PACS.

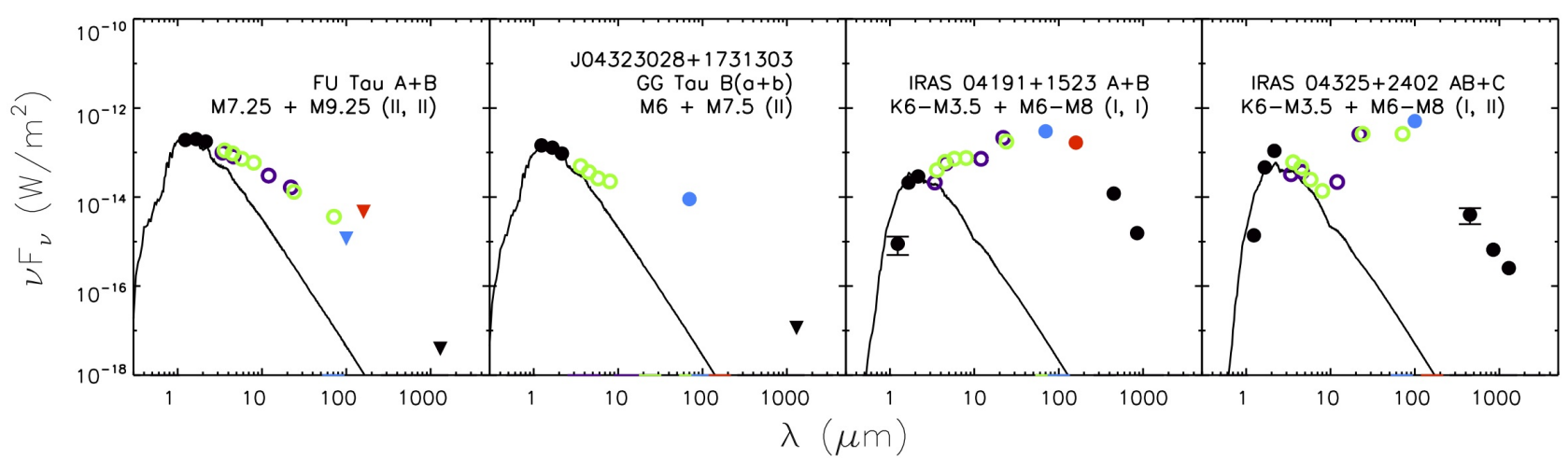

Fig. D.1. SEDs of the four known TBOSS Class I and/or Class II multiple systems for which the system broadband photometry and the combined atmospheric models of each system component are shown. The system name, component spectral types, and spectral classes are labeled in each SED. The observed broadband photometry is compiled from optical $\left(R_{\mathrm{C}}, I_{\mathrm{C}}\right)$, and near-IR (2MASS; $\left.J H K_{\mathrm{S}}\right)$ wavelengths (black points), the mid-IR (IRAC and WISE; green and purple open circles respectively), the far-IR (MIPS, PACS blue and red channels; green open circles, blue and red points respectively) and submm-mm wavelengths (black points). $3 \sigma$ upper limits are represented by the downwards triangles. The best-fit atmospheric model are displayed for each target. 


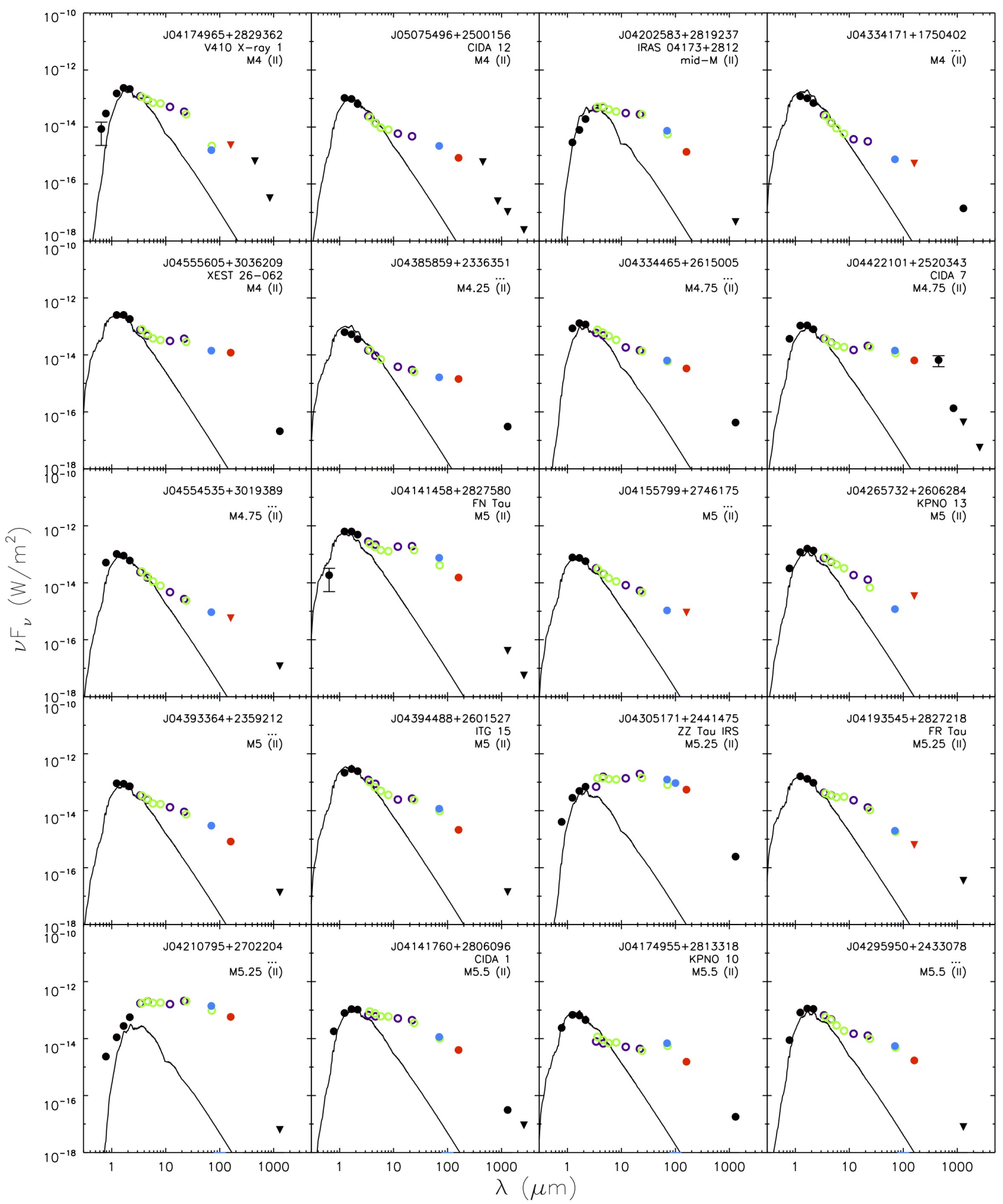

Fig. D.2. SEDs of the detected Class II objects with spectral types M4-M5.5. The target name, spectral type, and spectral class are labeled in each SED. The observed broadband photometry is compiled from optical $\left(R_{\mathrm{C}}, I_{\mathrm{C}}\right)$, and near-IR $\left(2 \mathrm{MASS} ; J H K_{\mathrm{S}}\right)$ wavelengths (black points), the mid-IR (IRAC and WISE; green and purple open circles respectively), the far-IR (MIPS, PACS blue and red channels; green open circles, blue and red points respectively) and submm-mm wavelengths (black points). $3 \sigma$ upper limits are represented by the downwards triangles. The best-fit atmospheric model are displayed for each target. 
J. Bulger et al.: Disk emission across the stellar/substellar boundary in Taurus

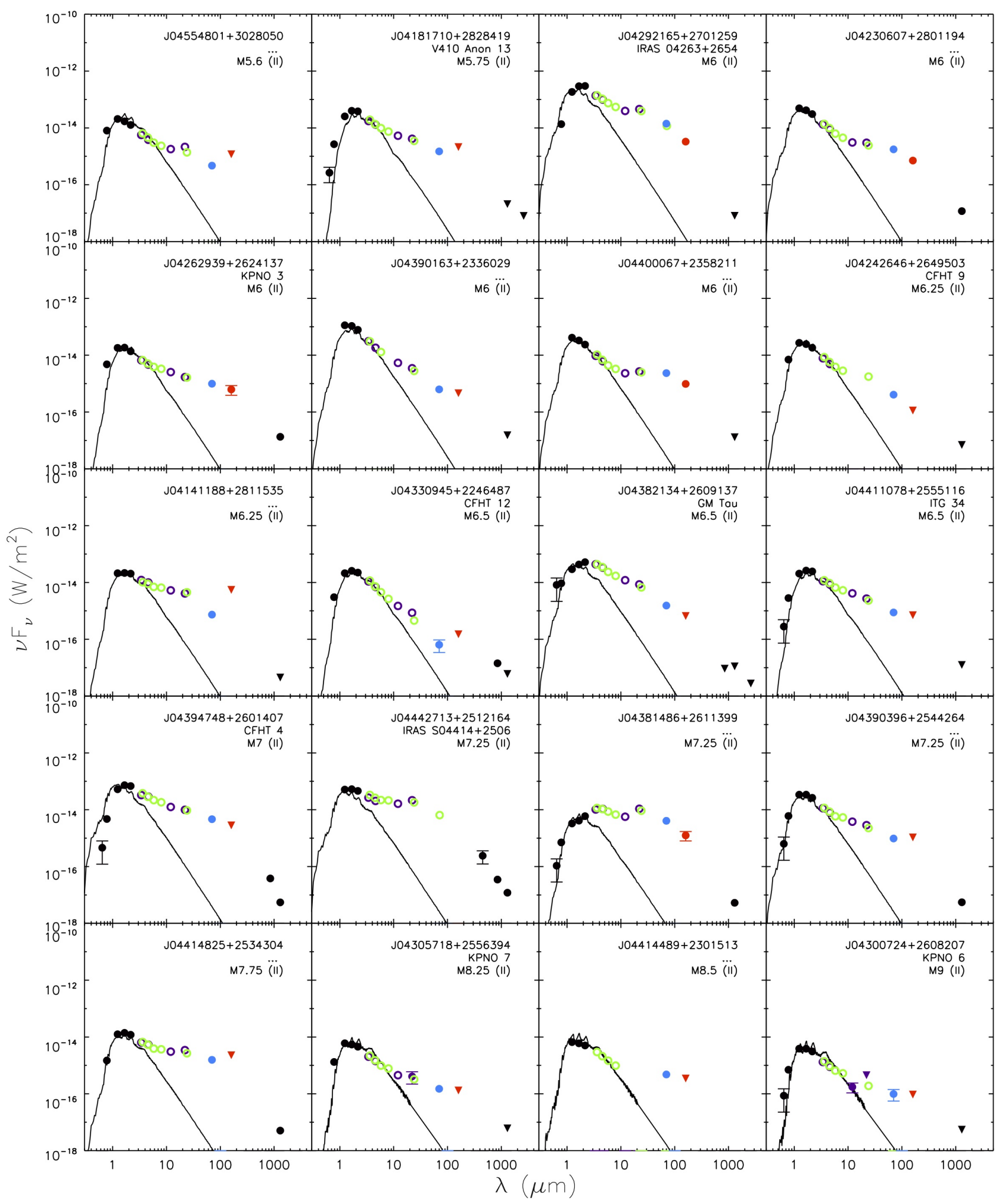

Fig. D.3. SEDs of the detected Class II objects with spectral types M5.6-M9. The target name, spectral type, and spectral class are labeled in each SED. The observed broadband photometry is compiled from optical $\left(R_{\mathrm{C}}, I_{\mathrm{C}}\right)$, and near-IR (2MASS; $\left.J H K_{\mathrm{S}}\right)$ wavelengths (black points), the mid-IR (IRAC and WISE; green and purple open circles respectively), the far-IR (MIPS, PACS blue and red channels; green open circles, blue and red points respectively) and submm-mm wavelengths (black points). $3 \sigma$ upper limits are represented by the downwards triangles. The best-fit atmospheric model are displayed for each target. 
A\&A 570, A29 (2014)

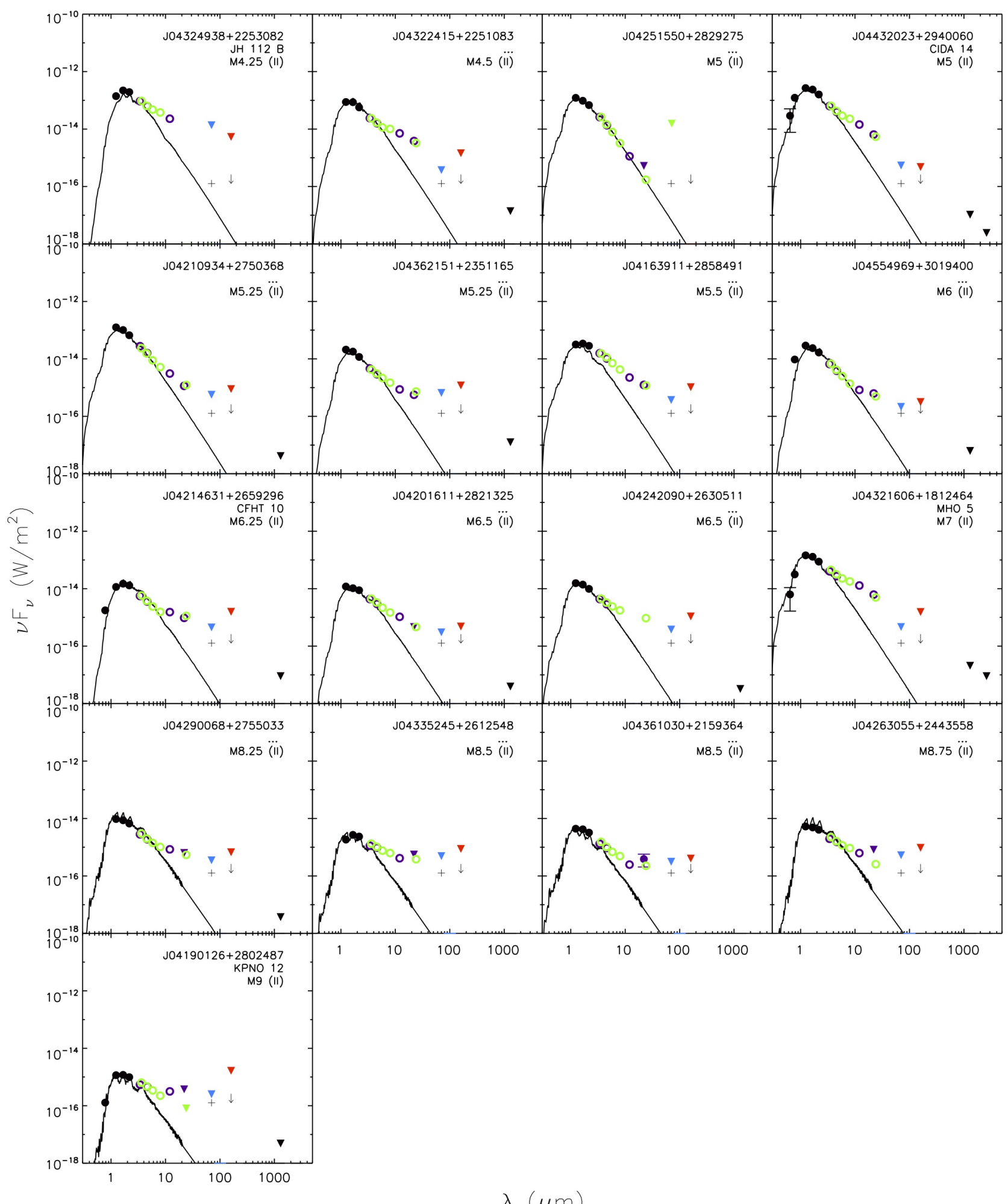

$\lambda(\mu \mathrm{m})$

Fig. D.4. SEDs of the undetected Class II objects with spectral types spanning M4.25-M9. The target name, spectral type, and spectral class are labeled in each SED. The observed broadband photometry is compiled from optical $\left(R_{\mathrm{C}}, I_{\mathrm{C}}\right)$, and near-IR $\left(2 \mathrm{MASS} ; J H K_{\mathrm{S}}\right)$ wavelengths (black points), the mid-IR (IRAC and WISE; green and purple open circles respectively), the far-IR (MIPS, PACS blue and red channels; green open circles, blue and red points respectively) and submm-mm wavelengths (black points). $3 \sigma$ upper limits are represented by the downwards triangles. The best-fit atmospheric model are displayed for each target. The gray cross shows level of the artificial detection generated from combined Class II $(70 \mu \mathrm{m})$ upper limit maps, and similarly the gray downward arrow shows the $160 \mu \mathrm{m}$ upper limit from the combined maps. 


\section{Appendix E: SEDs of the TBOSS sample - Class III objects}

The SEDs for the Class III objects of the TBOSS sample that are undetected with Herschel PACS are shown in Figs. E.1-E.4.

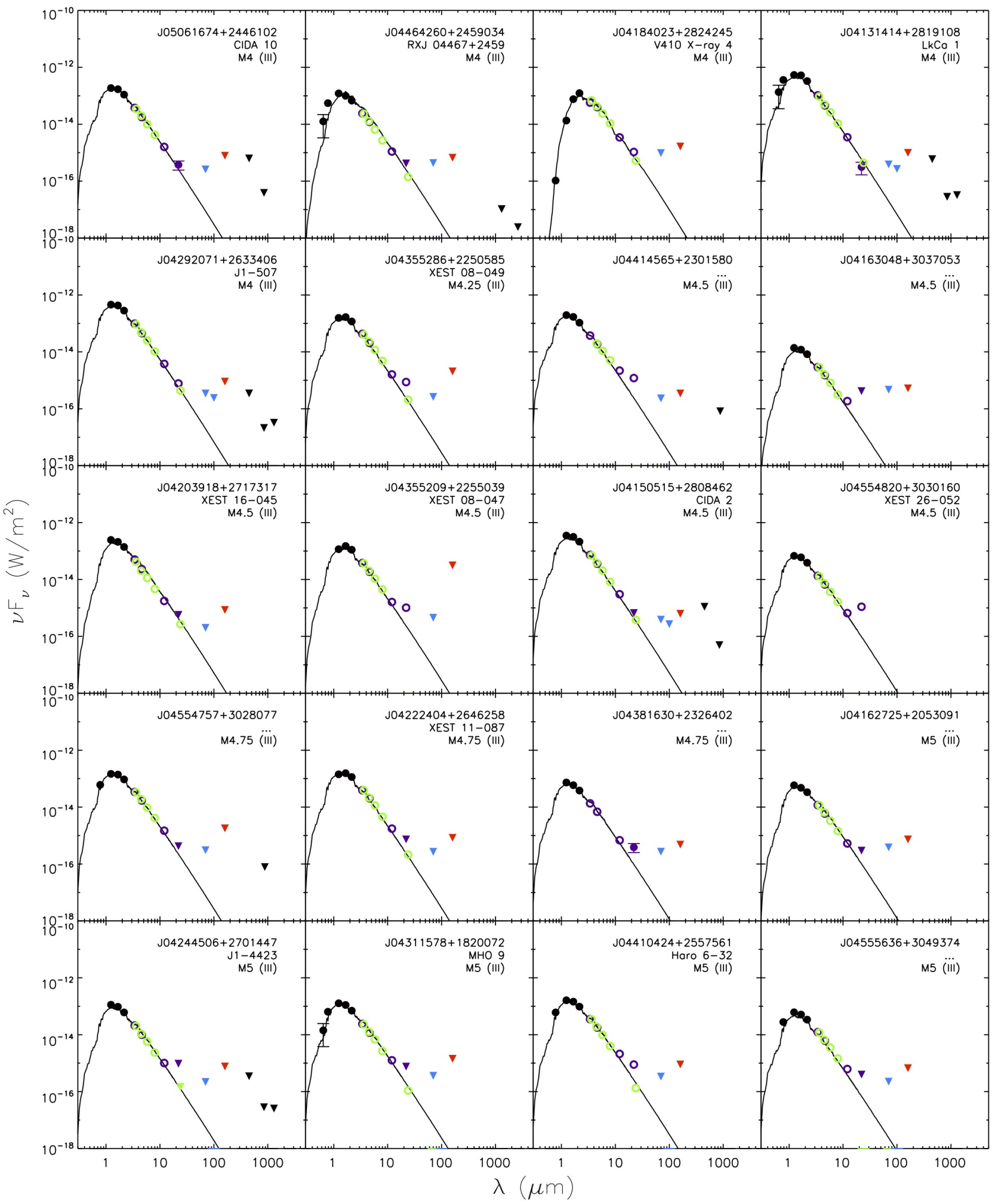

Fig. E.1. SEDs of the undetected Class III objects with spectral types spanning M4-M5. Additionally, the known M4.5 member - XEST 26-052 for which no far-IR observations exist, is also included within the panel. The target name, spectral type, and spectral class are labeled in each SED. The observed broadband photometry is compiled from optical $\left(R_{\mathrm{C}}, I_{\mathrm{C}}\right)$, and near-IR (2MASS; $J H K_{\mathrm{S}}$ ) wavelengths (black points), the mid-IR (IRAC and WISE; green and purple open circles respectively), the far-IR (MIPS, PACS blue and red channels; green open circles, blue and red points respectively) and submm-mm wavelengths (black points). $3 \sigma$ upper limits are represented by the downwards triangles. The best-fit atmospheric model are displayed for each target. 


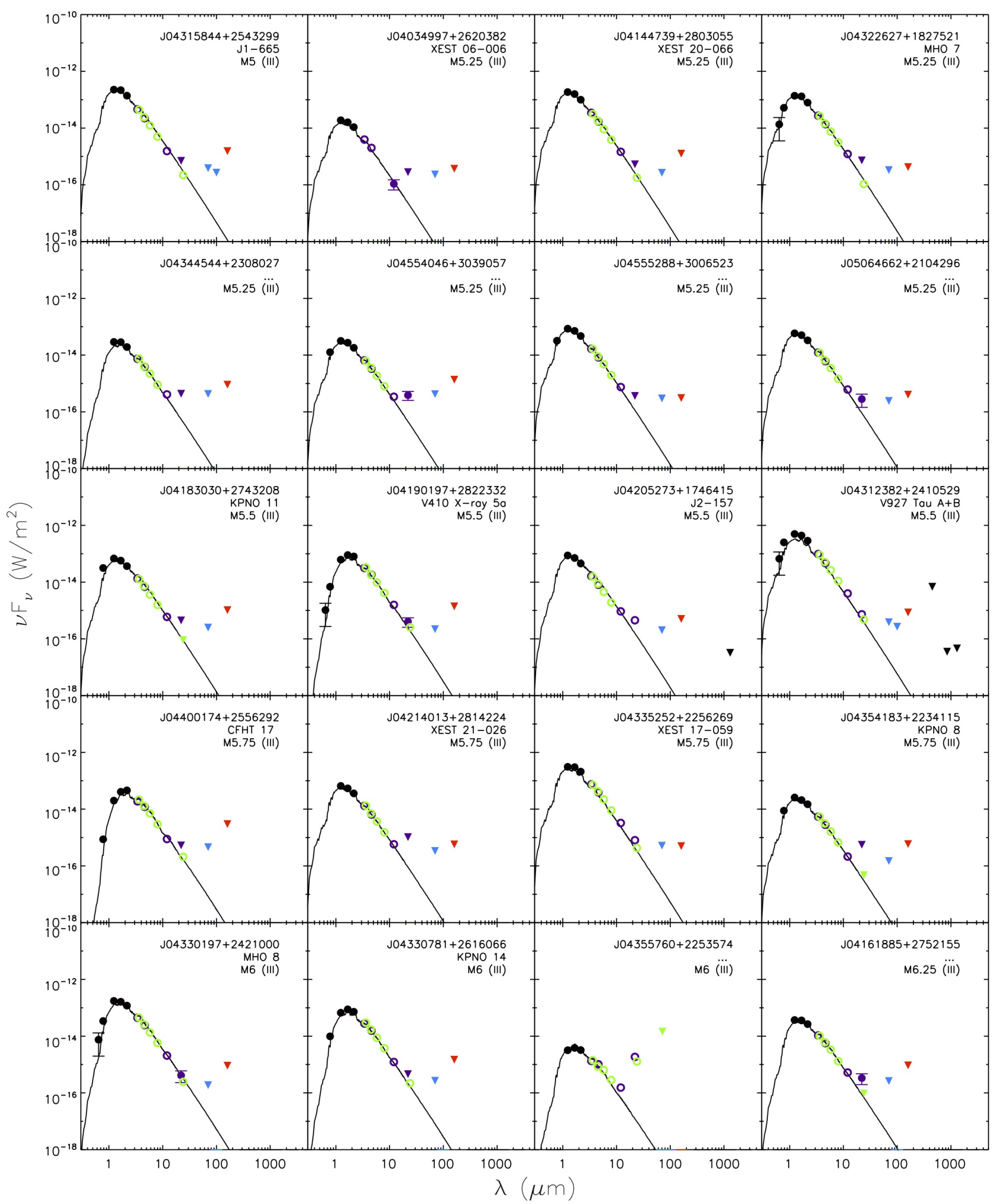

Fig. E.2. SEDs of the undetected Class III objects with spectral types spanning M5-M6.25. The target name, spectral type, and spectral class are labeled in each SED. The observed broadband photometry is compiled from optical $\left(R_{\mathrm{C}}, I_{\mathrm{C}}\right)$, and near-IR $\left(2 \mathrm{MASS} ; J H K_{\mathrm{S}}\right)$ wavelengths $($ black points), the mid-IR (IRAC and WISE; green and purple open circles respectively), the far-IR (MIPS, PACS blue and red channels; green open circles, blue and red points respectively) and submm-mm wavelengths (black points). $3 \sigma$ upper limits are represented by the downwards triangles. The best-fit atmospheric model are displayed for each target. 
J. Bulger et al.: Disk emission across the stellar/substellar boundary in Taurus

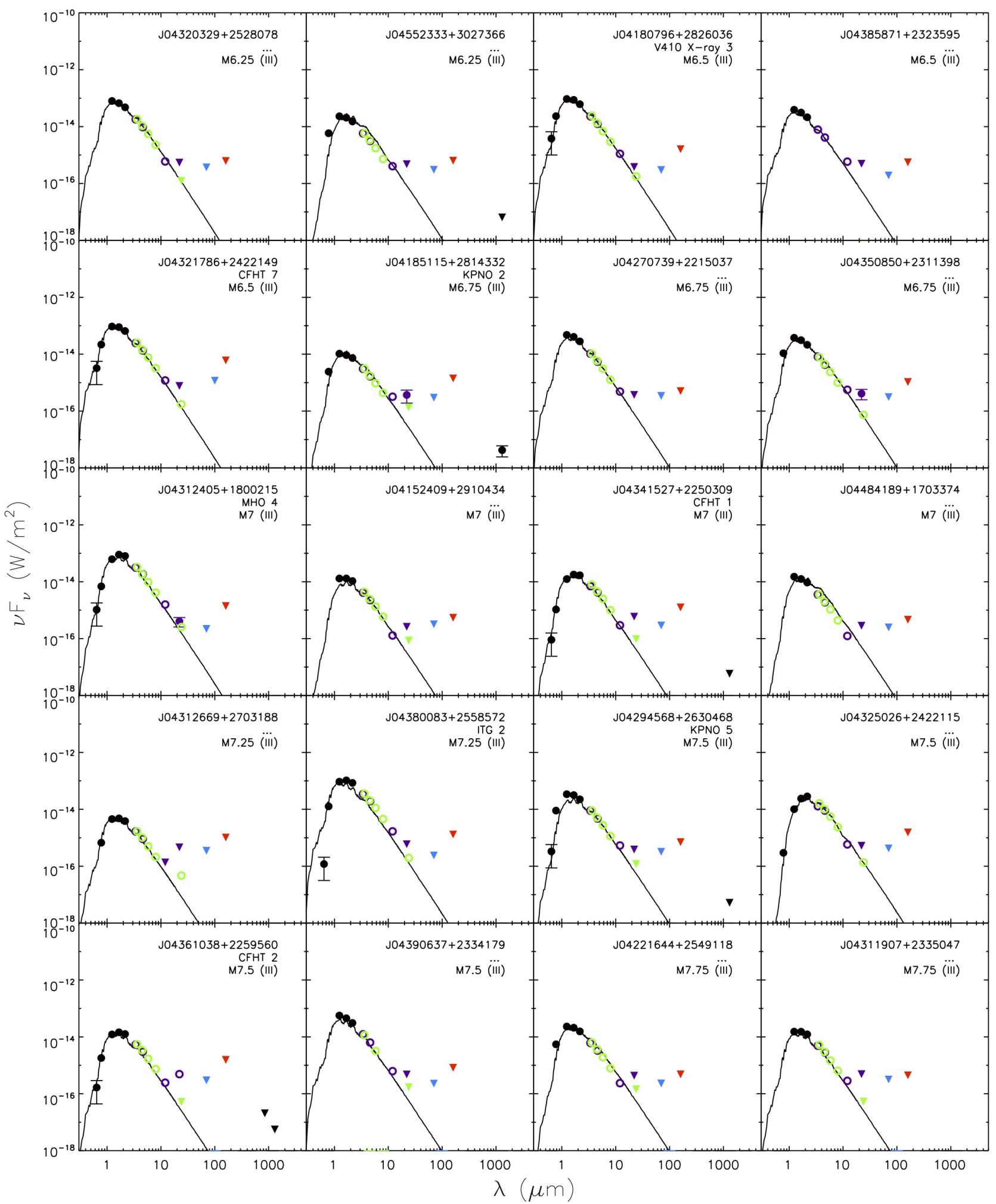

Fig. E.3. SEDs of the undetected Class III objects with spectral types spanning M6.25-M7.75. The target name, spectral type, and spectral class are labeled in each SED. The observed broadband photometry is compiled from optical $\left(R_{\mathrm{C}}, I_{\mathrm{C}}\right)$, and near-IR (2MASS; $\left.J H K_{\mathrm{S}}\right)$ wavelengths $($ black points), the mid-IR (IRAC and WISE; green and purple open circles respectively), the far-IR (MIPS, PACS blue and red channels; green open circles, blue and red points respectively) and submm-mm wavelengths (black points). $3 \sigma$ upper limits are represented by the downwards triangles. The best-fit atmospheric model are displayed for each target. 
A\&A 570, A29 (2014)

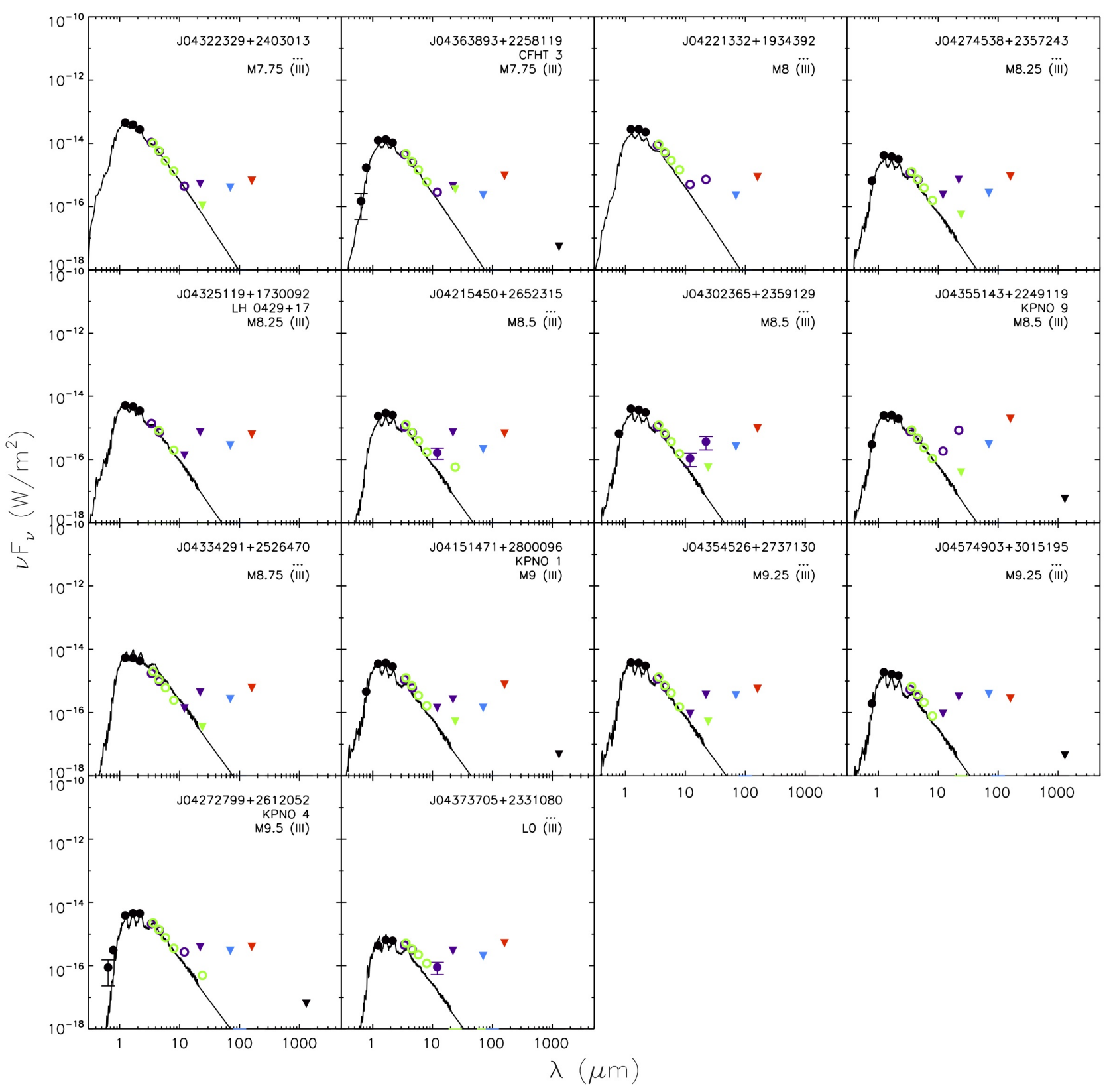

Fig. E.4. SEDs of the undetected Class III objects with spectral types spanning M7.75-L0. The target name, spectral type, and spectral class are labeled in each SED. The observed broadband photometry is compiled from optical $\left(R_{\mathrm{C}}, I_{\mathrm{C}}\right)$, and near-IR (2MASS; $\left.J H K_{\mathrm{S}}\right)$ wavelengths (black points), the mid-IR (IRAC and WISE; green and purple open circles respectively), the far-IR (MIPS, PACS blue and red channels; green open circles, blue and red points respectively) and submm-mm wavelengths (black points). $3 \sigma$ upper limits are represented by the downwards triangles. The best-fit atmospheric model are displayed for each target. 